CENTRO DE INVESTIGACION Y DE

ESTUDIOS AVANZADOS DEL IPN

\title{
MEASUREMENT OF THE
}

$J / \Psi$ PHOTOPRODUCTION

CROSS SECTION

by

Héctor Méndez

A Dissertation submitted to the Department of Physics in partial fulfillment of the requirements for the degree of Doctor of Science

Mexico, D.F.

April, 1990 
A mi Esposa Celina, A mi Madre Yolanda, A mis Hermanas y Hermano Brigida, Mabel, Jaqueline y Rafael 


\section{Abstract}

The elastic $J / \Psi$ photoproduction cross section on beryllium has been measured for photon energies up to $400 \mathrm{GeV}$. We discuss the experimental and analysis techniques used to obtain the sample of events and to calculate the cross section. Measurement of the energy dependence of the cross section is presented. The results are compared with data from previous experiments and with the predictions from current QCD models of $J / \Psi$ photoproduction. The presented data was taken from E-687 fixed target experiment at Fermilab in 1987 and at the beginning of 1988. 


\section{Acknowledgments}

The first day of my arrival to Fermilab was very pleasant, it was warm, the trees were green and the sky was blue. During the next few days, however, it was so cold, so windy and I was so impressed with the size of the E687 spectrometer, in particular, with the huge size of M2, under construction at that time. Then, my first experience at Fermilab was shocking, somewhat frightening but challenging at the same time. The long process of understanding, study and work at E687 turned out to be one of the most important experiences in my life. I wish to take this opportunity to express my gratitude to the many people without whose assistance the work described in this thesis could not have been performed.

I would like to thank Fermilab Director for giving me the opportunity, under very special circumstances, to be a part of one of the collaborating individuals of the experiment.

It is a pleasure to express my sincere gratitude and admiration to Dr. Joel Butler for giving me the opportunity to work in E687, for his advice during my stay at Fermilab, and for teaching me the many details involved in the experiment.

I sincerely thank my colleague and friend Dr. Angel Lopez for his guidance and support throughout the years and specially from whom I learned many details of the experiment as well.

I gratefully acknowledge the efforts of all the many people who have contributed to the success of E687 experiment. I wish to thank my fellow graduate students for sharing the hard work, in particular, Rik Yoshida who compile much of the muon data, Steve Culy and Seongwan Park for the development of the Monte Carlo program.

I also want to thank Dr. Carlos Hojvat for his much appreciated help and John Cumalat, Matias Moreno, Jerry Busenitz and Marco Giammarchi for the many useful discussions and corrections made on this thesis.

I would like to thank my teachers in Mexico, specially Miguel Angel Perez, Arnulfo Zepeda, Miguel Socolovsky and Enrique Campesino from whom I received much encouragement and support while I was a student at Cinvestav.

My appreciation also extends to my friends and compañeros Mario Venegas, Patricio Alamos and Lilian Ferrer from whom I received much support while I was living in Ilinois.

A very special thanks go to my compañera and wife Celina from whom I received much help and love.

To all of them, thank you very "verry" much and Muchas Gracias. 


\section{Contents}

Abstract

Acknowledgments

List of Figures v v

List of Tables vii

Introduction 1

1 Theory 3

1.1 Introduction ..................... 3

1.2 Vector Meson Dominance .............. 4

1.3 Photon Gluon Fusion . . . . . . . . . . . . . . 6

2 Experimental Setup $\quad 10$

2.1 Introduction . . . . . . . . . . . . . . 10

2.2 Beam line . . . . . . . . . . . . . . . . . 11

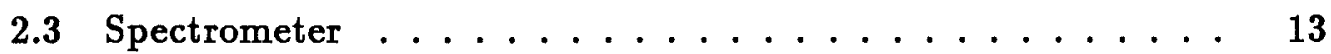

2.4 Trigger .............................. 20

3 Muon System Calibration $\quad 24$

3.1 Introduction . . . . . . . . . . . . . . . . . . 24

3.2 Muon System Setup ................. 25

3.3 Counter Geometry ................. 27

3.4 Multiple Scattering Correction . . . . . . . . . . 30

3.5 Magnetic Correction . . . . . . . . . . . . . . . 37

3.6 Proportional Tubes Geometry . . . . . . . . . . . . . 42

3.7 Efficiency Measurements ... . . . . . . . . . . . 45

3.8 Muon Identification . . . . . . . . . . . . . . 46 
4 Data Analysis and Conclusions 53

4.1 Introduction . . . . . . . . . . . . . . 53

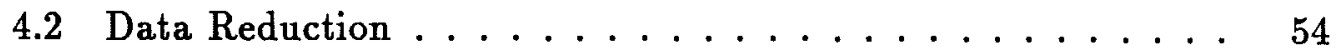

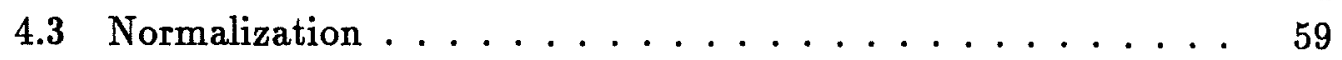

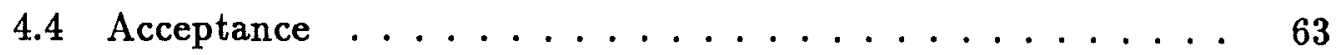

4.5 Results and Conclusions .................. 68

$\begin{array}{ll}\text { Bibliography } & 76\end{array}$ 


\section{List of Figures}

1.1 Vector Meson Dominance Model . . . . . . . . . . . . 5

1.2 a)Photon Gluon Fusion Process b)Bethe-Heitler Process . . . 7

1.3 Predicted $J / \Psi$ Cross Section by PGF . . . . . . . . . . . 9

2.1 Fermilab Site . . . . . . . . . . . . . . 12

2.2 Primary Target . . . . . . . . . . . . . . . 12

2.3 Photon Beam ................... 13

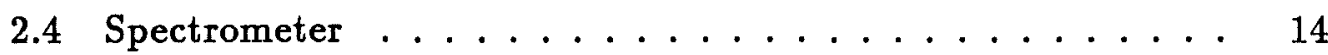

2.5 SSD Detector . . . . . . . . . . . . . . 16

$2.6 \mathrm{OE}$ and IE Detector . . . . . . . . . . . . 18

2.7 HC Detector . . . . . . . . . . . . . . . 19

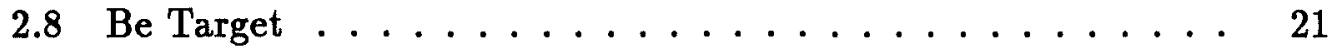

$2.9 H \times V$ and $O H$ Counters .............. 22

3.1 Spectrometer . . . . . . . . . . . . . . . 26

3.2 Muon System Setup .................. 26

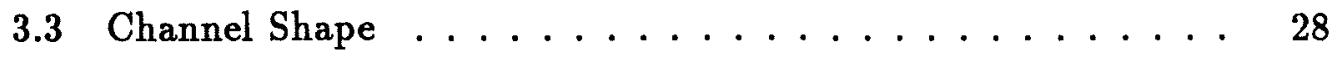

3.4 Projection .......................... 29

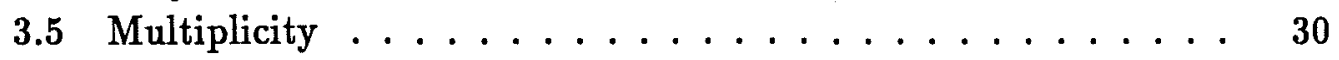

3.6 Multiple Scattering Diagram . . . . . . . . . . . 31

3.7 Residuals . . . . . . . . . . . . . . . . . 32

3.8 Multiple Scattering Data . . . . . . . . . . . . 33

3.9 Multiple Scattering Fit . . . . . . . . . . . 35

3.10 M2 Geometry . . . . . . . . . . . . . . . 38

3.11 M2 Field . . . . . . . . . . . . . . . . . 40

3.12 Inefficiency . . . . . . . . . . . . . . . . . . . 47

3.13 Muon Trigger $J / \Psi$ Sample (Bin of $40 \mathrm{MeV}$ ) . . . . . . . . . 49

3.14 Muon Identification Sample (Bin of $100 \mathrm{MeV}$ ) . . . . . . . 50

3.15 Muon Id Efficiency .................. . . . 51

3.16 Muon Data Selection Results .............. 52 
$4.1 \Delta E=E_{\gamma}-E_{J / \Psi} \ldots \ldots \ldots \ldots \ldots$

$4.2 \quad J / \Psi$ Candidates Sample . . . . . . . . . . . . . . . 57

$4.3 \quad J / \Psi \quad t\left(=P_{T}^{2}\right)$ Distribution . . . . . . . . . . . . 57

$4.4 \mathrm{~J} / \Psi$ Energy Distribution . . . . . . . . . . . . . . 58

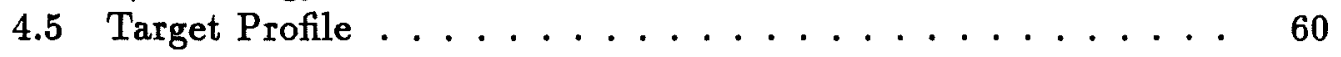

4.6 Photon Beam Generated .............. . . 61

4.7 BGM Vs. SEM . . . . . . . . . . . . . . . . . 62

4.8 "Good Spills" . . . . . . . . . . . . . . . . 62

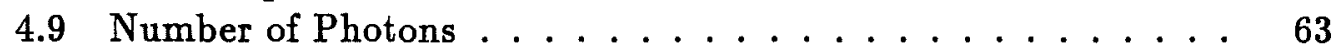

4.10 Total Number of $p, e, \gamma$ and $J / \Psi$ per Run . . . . . . . 64

4.11 Decay Angular Distribution .............. 66

$4.12 \Delta P=P_{G e n}-P_{\text {Rec }} \ldots \ldots \ldots$. . . . . . . . . 67

4.13 Total Acceptance As a Function of $t \ldots \ldots 67$

4.14 Acceptance ..................... 69

4.15 Total Acceptance as a Function of $E_{\gamma} \ldots \ldots \ldots$

4.16 Fit of $t$ Distribution . . . . . . . . . . . . . . . 71

$4.17 \mathrm{~J} / \Psi$ Cross Section per Nucleon . . . . . . . . . . . 73

4.18 Experimental and Theoretical $J / \Psi$ Cross Section . . . . . 74 


\section{List of Tables}

2.1 Multiwire Chamber Setup . . . . . . . . . . . 15

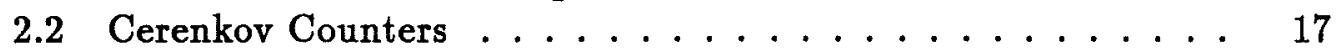

2.3 Target Setup .................. 20

3.1 Muon System Geometry . . . . . . . . . . . . 27

3.2 Counter Planes Shift ................. 29

3.3 Upstream Detector of Inner System . . . . . . . . . 36

3.4 Analytical "Inner" Calculation . . . . . . . . . . . 37

3.5 Upstream Detector of Outer System . . . . . . . . . . . . . 42

3.6 Analytical "Outer" Calculation .............. . . 42

3.7 Inner Muon Proportional Tubes Corrections . . . . . . . . . 45

3.8 Proportional Tube Planes Shift . . . . . . . . . . . 45

3.9 Average Efficiency . . . . . . . . . . . . . . 46

3.10 Probability of Missing Planes ... . . . . . . . . . 48

3.11 Probability of Matching . . . . . . . . . . . . . 49

$4.1 \quad J / \Psi$ Sample Fit . . . . . . . . . . . . . . . 56

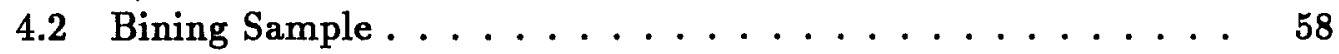

$4.3 \mathrm{~J} / \Psi$ Cross Section per Nucleus $\ldots \ldots \ldots$ 


\section{Introduction}

The E-687 fixed target experiment is designed to study "High Energy Photoproduction of States Containing Heavy Quarks". The experiment is being performed in the Wide Band Photon Laboratory at Fermi National Accelerator Laboratory (Fermilab). E-687 is a large group of collaborating institutions: University of Colorado, Fermilab, University of Illinois, INFN Bologna, INFN Frascati National Laboratories, INFN Milano, Northwestern University, University of Notre Dame and INFN Pavia. The experiment was proposed in January 31, 1981 and was approved in 1982. The first run began in the fall of 1987 being interrupted for almost 2 months due to an unfortunate accident when an electromagnetic calorimeter caught fire causing damage to other detectors. The analysis presented in this report corresponds to the postfire data.

Some of the goals of the experiment include the measurement of the open and bound charm photoproduction cross section, $D^{0}, D_{s}, D^{*}, J / \Psi$, etc., as well as the measurement of open and bound bottom cross section, i.e., $B, \Upsilon$, etc. Measurement of the lifetime of charm baryons $\left(\Lambda_{c}\right)$. Measurement of branching ratios of the final states of the observed meson.

To achieve these goals, high sensitivity to low cross sections, it was necessary to build a beam line adapted for the purposes of high photon intensity and a spectrometer capable of detecting multi particle events. The photon beam is obtained by bremsstrahlung when the electron beam, produced by a proton beam of $800 \mathrm{GeV}$, passes through the radiator producing a photon beam which is directed to the experimental target. The intensity of the beam is around $10 \times 10^{6}$ photons/spill with energies greater than $133 \mathrm{GeV}$. The spectrometer is $32 \mathrm{~m}$ long and consists basically of a tracking system (2 analysis magnets, multiwire proportional chambers and microstrip detector), a particle identification system (calorimeters, muon and cerenkov counters) and beam monitoring detectors. The data acquisition system, activated by a 
simple trigger, consists essentially of 5 Fastbus memories which collect a big fraction of the total hadronic cross section.

In this report we present the measurement of the $J / \Psi$ elastic cross section extracted from the dimuon final state. In order to make this measurements possible, one must have a good understanding of the above mentioned topics. In chapter 1 of this report we study the theoretical predictions by Quantum Chromodynamics for the $J / \Psi$ cross section. Chapter 2 describes the main characteristics of the photon beam and the spectrometer. Chapter 3 gives a full description of the muon system and a study of the muon identification criterion. Finally, in chapter 4 we present the measurement of the $J / \Psi$ cross section and conclude by comparing these results with previous experimental data and with the theoretical predictions. 


\section{Chapter 1}

\section{Theory}

\section{$1.1 \quad$ Introduction}

Considerable attention in the heavy quark physics was originated with the discovery of the $J / \Psi$. In November 1974, two independent experiments (proton-Be collisions [1] and $e^{-} e^{+}$interactions [2]) reported a narrow $(\Gamma=63 \mathrm{KeV}$ ) resonance ( $J$ or $\Psi$ ) with a mass of $3.096 \mathrm{GeV}$ coupled electromagnetically to leptons. Several authors suggested that this new particle can be identified as the lowest mass vector meson associated with a new quark predicted during the early 1960's [3] in the four quark theory. This new quark, carrying a charge $Q_{c}=+2 / 3$ and a new quantum number, charm (c), (conserved in strong and electromagnetic interactions and violated in weak interactions) explains the suppression of strangeness changing neutral currents [4] in the Weinberg-Salam model $[5,6]$ of electro-weak interactions. Strong evidence was collected over the next few months to identify the $J / \Psi$ as a bound state of $c \bar{c}$ quarks. In addition, the observation of another resonance $\left(\Psi^{\prime}\right)[8]$ was observed at $3.7 \mathrm{GeV}$ and is interpreted as an excited state of the heavy quark $c \bar{c}$ system.

From 1974 many experiments have been investigating the spectroscopy and production mechanism of the charmed particles. For example, SPEAR $[9,10]$ measured the $J^{P C}\left(1^{--}\right)$quantum number of the $J / \Psi$ and $\Psi^{\prime}$. Other experiments $[11,12,13]$ showed that the $J / \Psi$ interacted with nucleons at a level characteristic of a hadron.

Many groups have reported states showing the charm quantum number in $e^{-} e^{+}, \nu$, and $\gamma$-nucleon interactions. As photons couple to charm quark through its electric charge, the rate of bound and open charm production is proportional to $\left[Q_{c} / E_{c m}\right]^{2}$ in $e^{-} e^{+}$and $\left[Q_{c} / M_{c}\right]^{2}$ in $\gamma-n u c l e o n$ interactions. Approximately $40 \%$ of the $e^{-} e^{+}$and $1 \%$ of the photoproduction total cross sec- 
tion contains charmed particles in the final state. The relative level of charm production in hadron interaction is an order of magnitude lower. The absolute charm production rates are orders of magnitude higher in photon beam even though the background level is much lower in $e^{-} e^{+}$machine. With photon beam we can simultaneously observe all the charm spectrum [14].

Over the years, the leptoproduction of vector meson $(\rho(770), \omega(783)$, $\phi(1020)$ ) has been explained with the Vector Meson Dominance (VMD) model [15] which gives a good qualitative physical picture of the process. However, it does not have the predictive power of a strong interaction theory such as the Quantum Chromodynamics (QCD). QCD gives us a simple and natural explanation of the production of hadrons by photons via the Photon Gluon Fusion (PGF) model introduced in 1978 [16]. Perturbative QCD computations yields accurate predictions when the mass $M^{2}$ is high enough so that the running coupling constant $\alpha_{a}$ is low.

In this chapter, we summarize the important aspects of the VMD and PGF approaches which are later compared to our results of the photoproduction of $J / \Psi$.

\subsection{Vector Meson Dominance}

The VMD model is the earliest theoretical model developed as an attempt to explain the photoproduction of hadrons. In this model, the photon interacts with hadrons by first converting itself into a vector meson $(v)$ which subsequently scatters off the target nucleus (figure 1.1). This interaction is expressed by the hadronic electromagnetic current which is connected to the vector meson [15]. Then, the photoproduction amplitude is related to the amplitude of the vector meson which scatters off the target by [22],

$$
T_{\gamma v}=\frac{e}{\gamma_{v}} T_{v v}
$$

where $e$ is the electric charge $\left(e^{2} / 4 \pi=\alpha \approx 1 / 137\right)$ and $\gamma_{v}$ the dimensionless coupling constant of the photon to the vector meson.

In this picture, the vector meson photoproduction is related to the vector meson elastic scattering by the amplitude

$$
T_{\gamma p \rightarrow v p}=\frac{e}{\gamma_{v}} T_{v p \rightarrow v p}
$$

for the total cross section

$$
\sigma_{\gamma p}=\frac{e^{2}}{\gamma_{v}^{2}} \sigma_{v p}
$$




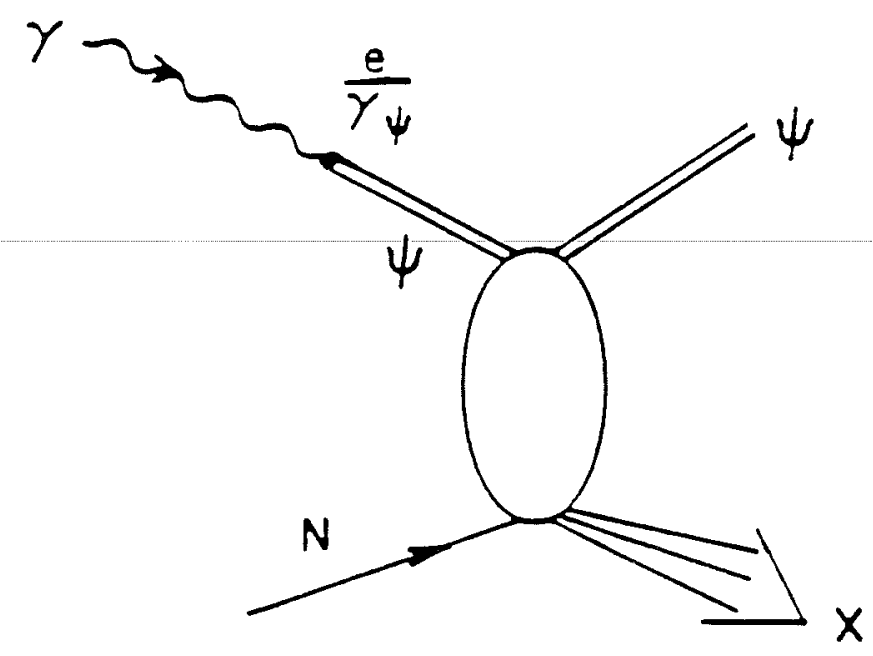

Figure 1.1: Vector Meson Dominance Model

or for the forward differential cross section

$$
\frac{d \sigma}{d t}(\gamma p \rightarrow v p)=\frac{e^{2}}{\gamma_{v}^{2}} \frac{d \sigma}{d t}(v p \rightarrow v p)
$$

Using the optical theorem, the elastic cross section is related to the total cross section $(\sigma)$

$$
\left.\frac{d \sigma^{e l}}{d t}\right|_{t=0}=\frac{\left(1+\eta^{2}\right)}{16 \pi} \sigma^{2}
$$

where $\eta$ is the ratio of the real to imaginary forward scattering amplitude. This result allow us to calculate the total hadronic photoproduction cross section by measuring the elastic meson photoproduction for each vector meson. The limit $t \rightarrow 0$ in equation 1.5 is not accessible experimentally. A minimum momentum transfer is required to make up the mass difference in the photon-vector meson transition. This is

$$
t_{\min }=-\frac{m_{v}^{4}}{\left(2 E_{\gamma}^{\text {lab }}\right)^{2}}
$$

where $m_{v}$ is the vector mass and $E_{\gamma}$ is the photon energy in the laboratory system. The zero momentum transfer cross section can be obtained by multiplying the differential cross section by the factor $e^{-b\left|t_{\min }\right|}$ where $b$ is a constant [17]. The coupling constant $\gamma_{v}$ can be determined if the vector meson nucleon total cross section is known; otherwise, it is still unknown. However, this parameter may also be calculated from the leptonic width $\left(\Gamma\left(v \rightarrow l^{+} l^{-}\right)\right)$of the 
vector meson. A simple $Q E D$ calculation gives the result

$$
\Gamma\left(v \rightarrow l^{+} l^{-}\right)=\frac{\alpha^{2}}{3} \frac{4 \pi}{\gamma_{v}^{2}} m_{v}\left(1-\frac{4 m_{l}^{2}}{m_{v}^{2}}\right)^{\frac{1}{2}}\left(1+\frac{2 m_{l}^{2}}{m_{v}^{2}}\right)
$$

where $m_{l}$ is the lepton mass. For a particular decay, $\Psi \rightarrow \mu^{+} \mu^{-}$, this expression turns into

$$
\Gamma\left(\Psi \rightarrow \mu^{+} \mu^{-}\right)=\frac{\alpha^{2}}{3} \frac{4 \pi}{\gamma_{\Psi}^{2}} m_{\Psi}
$$

A partial decay width is given in terms of the experimentally measured total decay width $\Gamma(v \rightarrow a l l)$,

$$
\Gamma\left(v \rightarrow l^{+} l^{-}\right)=B\left(l^{+} l^{-}\right) \Gamma(v \rightarrow a l l)
$$

where $B\left(l^{+} l^{-}\right)$is the measured branching ratio

$$
B\left(l^{+} l^{-}\right)=\frac{\sigma\left(v \rightarrow l^{+} l^{-}\right)}{\sigma(v \rightarrow a l l)}
$$

which are well measured in $e^{-} e^{+}$collision. Solving for $\Psi$ vector meson, the coupling constant is

$$
\frac{4 \pi}{\gamma_{\Psi}^{2}}=0.086 \pm 0.012
$$

With this knowledge of $\gamma_{\Psi}^{2}$, the $\Psi-$ nucleon elastic scattering cross section can be calculated through the relation 1.3 .

\subsection{Photon Gluon Fusion}

Much attention has been dedicated to the perturbative QCD predictions for heavy quark pair $(Q \bar{Q})$ production in high energy processes. A popular approach in photoproduction reactions is the photon-gluon fusion (PGF) model. The incident photon interacts with the gluon content of the target (proton) through the subprocess, $\gamma g \rightarrow Q \bar{Q}$, resulting in the production of the $Q \bar{Q}$ pair. Of course, at least one other gluon must be exchanged to conserve color. This color rearrangement is assumed to occur with probability one and not influence the validity of the calculation of the total cross section.

Figure 1.2 shows two process; a) photon-gluon fusion process and $b$ ) one similar (Bethe-Heitler) Quantum Electromagnetic (QED) process. Both process are related by substituting in the QED process the $e^{-} e^{+}$by the $Q \bar{Q}$ 


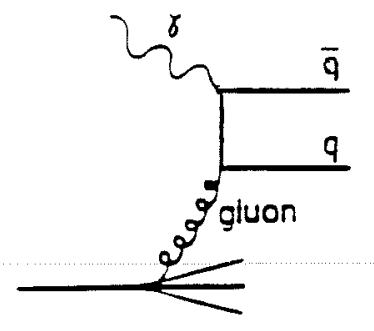

Q

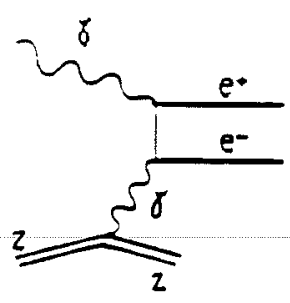

$b$

Figure 1.2: a)Photon Gluon Fusion Process b)Bethe-Heitler Process

pair, the exchanged photon by a gluon and the electromagnetic coupling constant $\alpha$ by the strong coupling constant $\alpha_{\mathbb{d}}$ in the quark-gluon vertex. $\alpha_{\theta}$ is a function of the mass under study $(m)$

$$
\alpha_{s}\left(m^{2}\right)=\frac{12 \pi}{\left(33-2 N_{f}\right) \ln \left(m^{2} / \Lambda^{2}\right)}
$$

with the number of flavor $N_{f}=4$ and the empirical scale parameter $\Lambda=$ $0.2 \mathrm{GeV} . \alpha_{\text {o }}$ is expected to be small enough to justify low order perturbation theory. In fact, for instance, $\alpha_{\bullet}$ for charm quark $\left(m_{c}=1.5 \mathrm{GeV}\right)$ is $\mathbf{0 . 3}$. The photon-gluon fusion is the lowest order QCD process.

Taking into account the quark charges and color factors, the gluon fusion cross section $\sigma_{\gamma g}$ is related to the electromagnetic cross section $\sigma_{\gamma \gamma \rightarrow Q Q}$ by

$$
\sigma_{\gamma g \rightarrow Q Q}=4 e_{Q}^{2} \frac{\alpha_{t}}{\alpha} \sigma_{\gamma \gamma \rightarrow Q Q}\left(M^{2}\right)
$$

where the cross section of the pair with mass $M$ is [18]

$$
\sigma_{\gamma \gamma \rightarrow Q Q}\left(M^{2}\right)=\frac{4 \pi \alpha^{2}}{M^{2}}\left[\left(1+y-\frac{y^{2}}{2}\right) \ln \left(\frac{1+\sqrt{1-y}}{1-\sqrt{1-y}}\right)-(1+y) \sqrt{1-y}\right]
$$

and for $M \gg M_{Q}$, we have

$$
\sigma_{\gamma \gamma \rightarrow Q Q}\left(M^{2}\right) \rightarrow \frac{4 \pi \alpha^{2}}{M^{2}} \ln \left(\frac{4}{y}\right)
$$

with $y=4 M_{Q}^{2} / M^{2}$ and $M_{Q}$ as the mass of the quark.

To compute the total cross section for producing a $Q \bar{Q}$ open or bound state, one must integrate the elementary cross section (equation 1.13) over the 
incident gluon momentum spectrum $F(x)$. This is,

$$
\sigma_{\gamma p \rightarrow Q \bar{Q}}=\int_{M_{t h}}^{M_{0}} \sigma_{\gamma g}\left(M^{2}\right) F\left(\frac{M^{2}}{s}\right) \frac{d M^{2}}{s}
$$

where $s=M_{p}^{2}+2 M_{p} E_{\gamma}^{l a b}$ is the squared total center of mass energy in the laboratory frame. The lower and upper integration limits are taken in the mass pair region being investigated. It is possible to compute this integral once we make the assumption that all bound $Q \bar{Q}$ states take places in the region $\left(2 M_{Q}^{2}\right)^{2} \leq M^{2} \leq\left(2 M_{l m}^{2}\right)^{2}$ and the open states in the region $M^{2} \geq\left(2 M_{l m}^{2}\right)^{2}$; where $M_{l m}$ is the mass of the lowest open flavor $Q$ production.

One step is necessary before carrying out this integration. It consists in the specification of the gluon distribution function $F(x)$. One form of $F(x)$ typically used is [19]

$$
F(x)=\frac{1}{16} \frac{(n+1)}{x}(1-x)^{n}
$$

which is normalized so that eight types of gluons together carry half the momentum of the proton. $n$ between 5 and 10 are the most used values [16].

For a $c \bar{c}$ bound production, as $J / \Psi$, the integral is done in the mass range between $\left(2 M_{c}-\frac{1}{2} \Gamma_{\text {tot }}^{J / \Psi}\right)^{2}$ and $\left(2 M_{D}\right)^{2}=(2 * 1.864)^{2}$. For this extremely narrow resonance, the total width $\Gamma_{\text {tot }}^{J / \Psi}$ can be safely neglected. Figure 1.3 show this calculation as a function of the photon energy for two different mass charm quark values. In 1977 Fritszch [20] pointed out that the cross section obtained by this method exceeded the measured cross section because there are many charmonium states in the mass interval $c \bar{c}$ system. Then, he suggested that semi-local duality [18] ideas should be applied to the estimation of any bound heavy quark system. This is, divide the calculated cross section by the total number of states in the $Q \bar{Q}$ bound states. In the $J / \Psi$ case, the number of bound states are 7 which is more or less the disagreement factor with the measured cross section. Nevertheless, the cross section measured at high photon energy seems to have a different shape with respect to the predicted one by this model.

In the last chapter of this report we compare our and other experiments data with the above predictions for $J / \Psi$ cross section. 


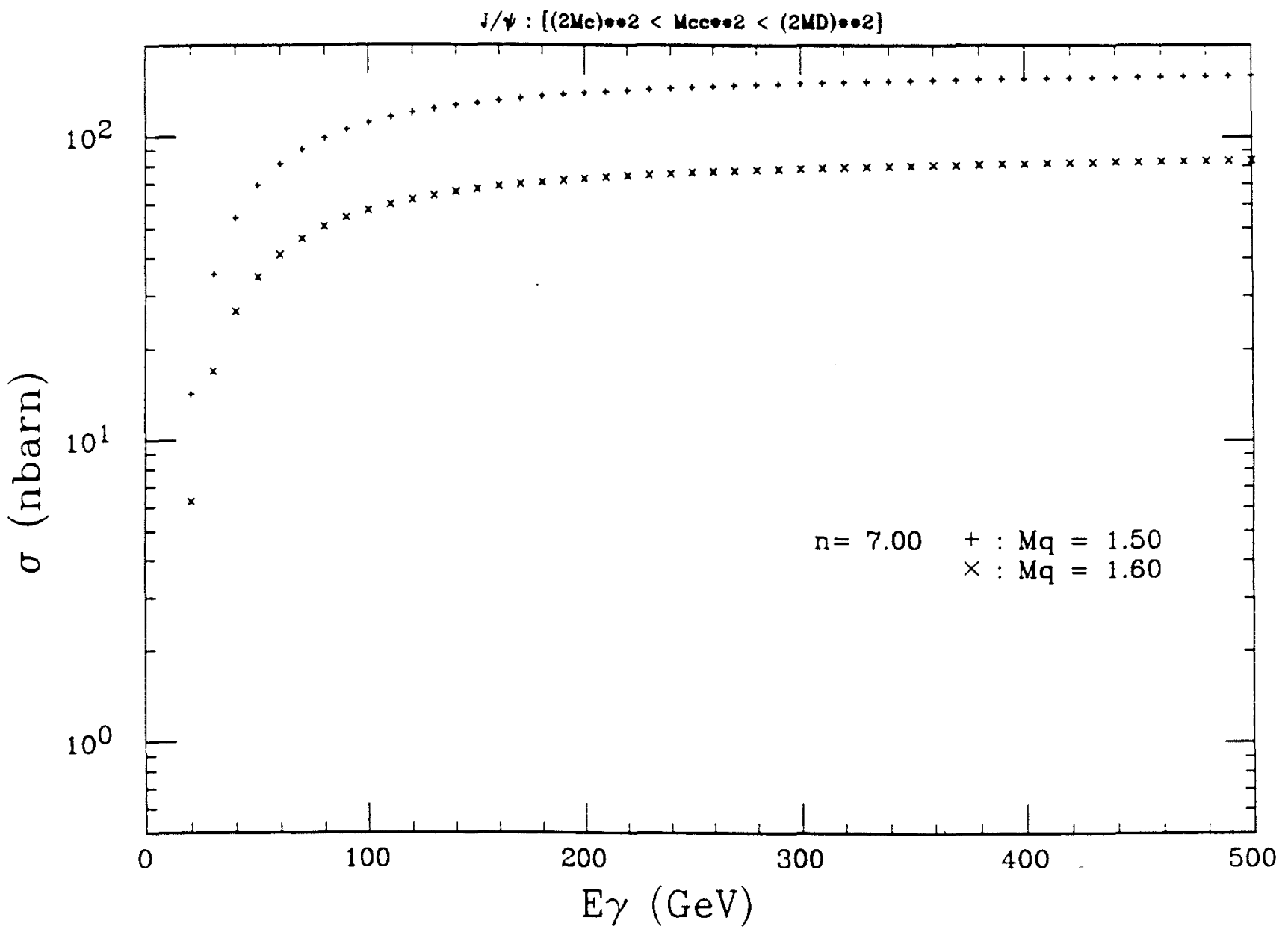

Figure 1.3: Predicted $J / \Psi$ Cross Section by PGF 


\section{Chapter 2}

\section{Experimental Setup}

\subsection{Introduction}

The E-687 experiment is designed to study "High Energy Photoproduction of States Containing Heavy Quarks". The experiment was performed in the Wide Band Photon Laboratory at Fermilab.

A proton beam of $800 \mathrm{GeV}$ from the accelerator is used to make an electron beam, which is directed to the Wide Band. A large energy spread photon beam is produced by bremsstrahlung when the electron beam goes through a lead radiator. The electrons are swept by magnets into a counter hodoscope (RESH) where their energy is measured and are stopped in a dump behind of the RESH counter. The photons with energies up to $450 \mathrm{GeV}$ go to the experimental target, in which states containing heavy quarks are produced by interactions with the components of the nuclei which make up the target.

When an event occurs, the data acquisition (DAQ) process starts if the trigger of the experiment is satisfied. The main trigger (master gate) consists of essentially the requirement of a minimum opening angle of the event, in order to reject pair production $\left(\gamma \rightarrow e^{+} e^{-}\right)$in the spectrometer. The multiparticle spectrometer is composed of a tracking system (two analyzing magnets,multiwire proportional chambers and microstrip detector), detectors identifying charged and neutral particles (muon counters, cerenkov counters and calorimeters), beam monitoring detectors and trigger/veto counters. The total number of channels in the spectrometer is approximately 30000 . The distance from the target to the end of the spectrometer is close to $32 \mathrm{~m}$.

A right handed reference system with center on the central analysis magnet had been used in the experiment. $Z$ is positive in the beam direction. In this chapter we described the main characteristics of the spectrometer, beam 
line and the triggers used in the experiment.

\subsection{Beam Line}

The photon beam used is generated from an incident proton beam. Since the proton beam at Fermilab has already been described in detail elsewhere [21], we give only a brief description of the four stages requires to accelerate it up to $800 \mathrm{GeV}$. The first stage is to strip away the electrons from Hydrogen atoms to provide a source of protons. Through the use of a Cockcroft Walton Accelerator, these protons are accelerated to $750 \mathrm{KeV}$. Next, protons are sent to a $200 \mathrm{MeV}$ Linear Accelerator (Linac) which injects the protons into the Booster Synchrotron. Thirteen successive "bunches" of protons are placed in the Booster and then accelerated by the Booster to $8 \mathrm{GeV}$. The protons are then injected into the Main Ring Accelerator (a proton synchrotron of $6.28 \mathrm{Km}$ of circumference and made of conventional magnets), which accelerates the beam to $150 \mathrm{GeV}$. Then, the last step of acceleration takes places in the Tevatron where the protons reach an energy peak of $800 \mathrm{GeV}$. The Tevatron is located under the main ring and is made up of superconducting magnets. The beam is extracted from the Tevatron in pulses of intensity $\approx 10^{13}$ protons for about $20 \mathrm{sec}$ out of each minute (spill). The extracted beam is directed to three experimental areas: Meson, Neutrino and Proton (figure 2.1). Once the beam is in the proton area, it is split again into three separate beams: Center, East and West. Next, the east line is split into two beams, in one of which this experiment is performed.

The method used to produce a photon beam starting from an incident proton beam had been well described in [22]. The proton beam (east line) is focussed on a beryllium target (figure 2.2) producing a large number of particles, including photons coming from $\pi^{0}$ 's produced in the reaction $p+$ $p \rightarrow \pi^{0}+X$. The unwanted charged particles are bent into a beam dump by sweeping magnets. The resulting neutral beam contains about $48 \%$ of neutrons, $48 \%$ of photons, $2 \%$ of $K_{L}^{0}$ 's and other neutral particles. Then, the neutral beam passes through a lead foil and the photons are converted to $e^{-} e^{+}$pairs. The unconverted photons and the neutral hadrons move forward at zero degrees and are absorbed in a neutral dump. By using a conventional beam line, ONLY the electrons are captured and then transported around the neutral dump to the Wide Band line (figure 2.3), which is located at zero net deviation from the direction of the incident proton beam.

Once we have the electron beam with energy $E_{0}$ in the wide band, it goes through a thin lead foil (radiator) to produce photons by bremsstrahlung. 


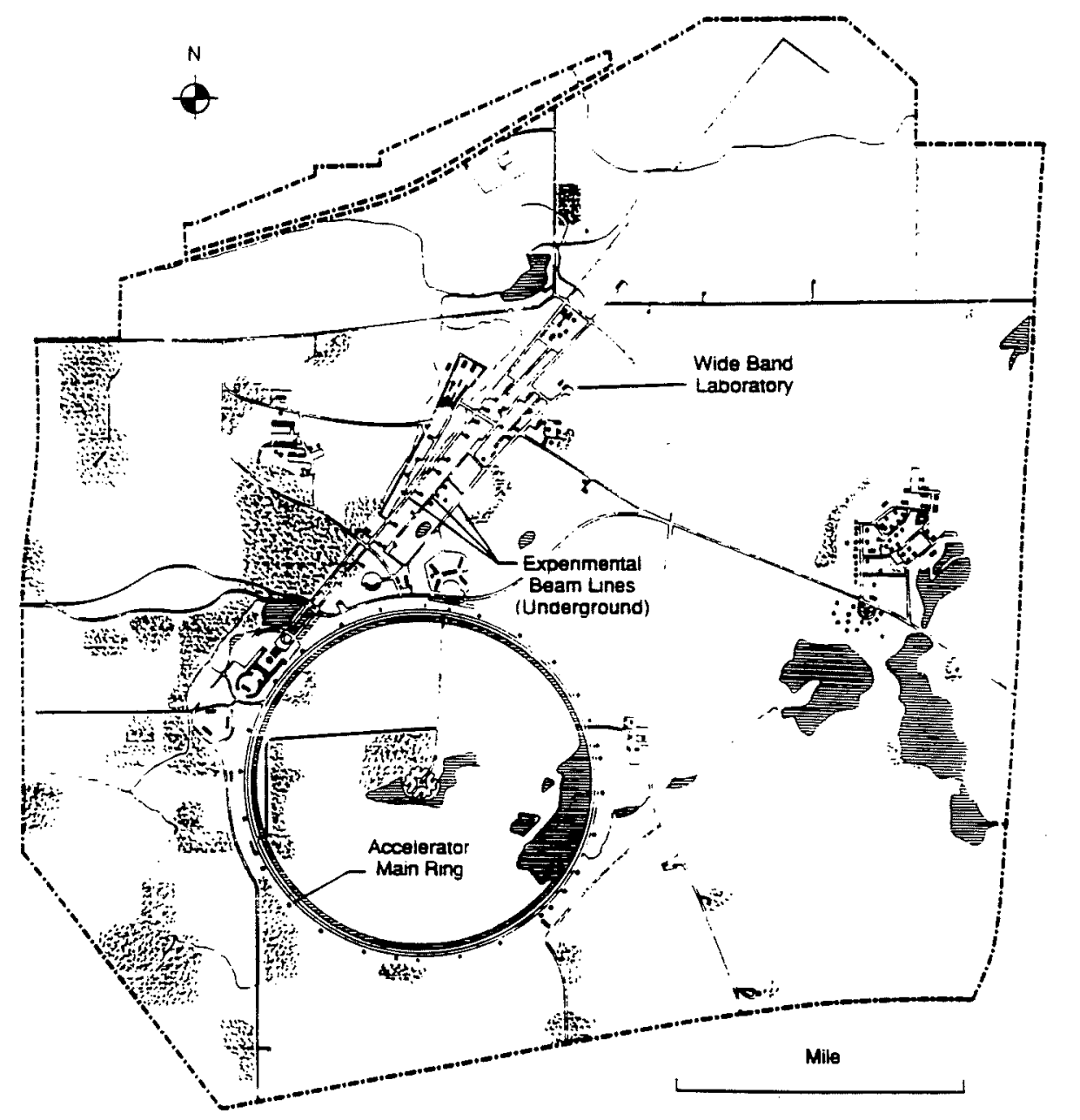

Figure 2.1: Fermilab Site

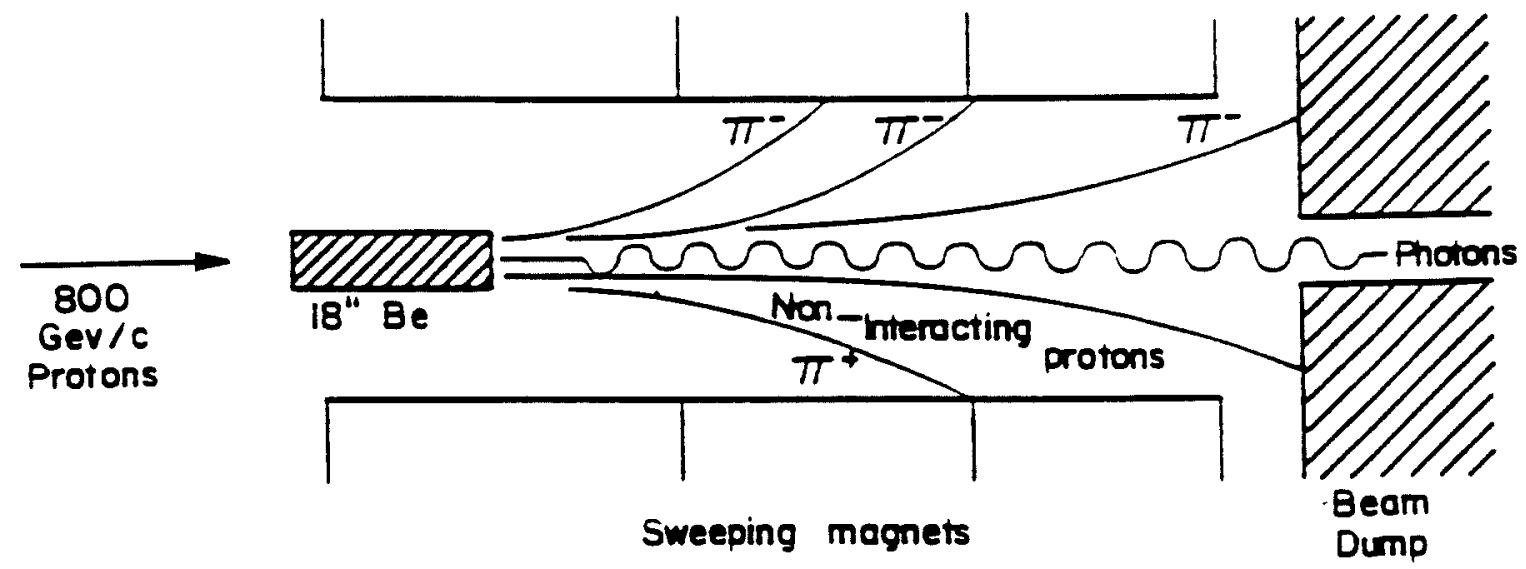

Figure 2.2: Primary Target 


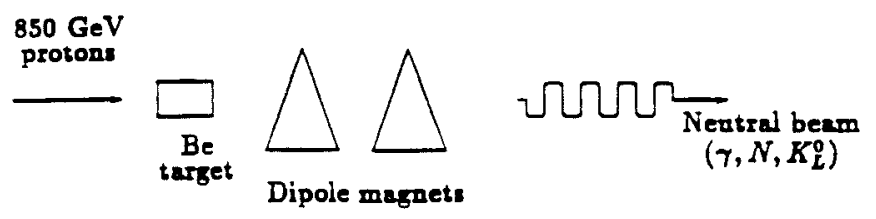

Step 2: Photon converuion

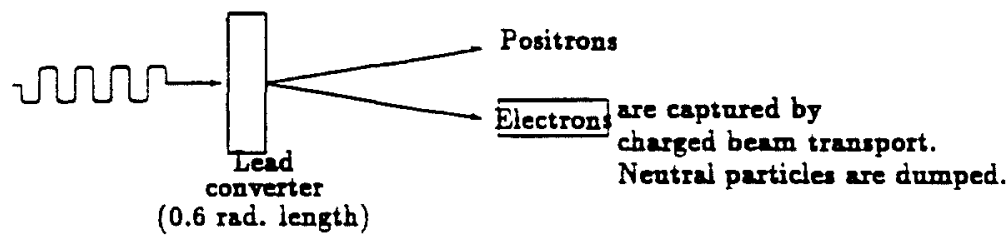

Step 3: Capture electrons in charged beam transport

Step 4: Radiate photons from electrons
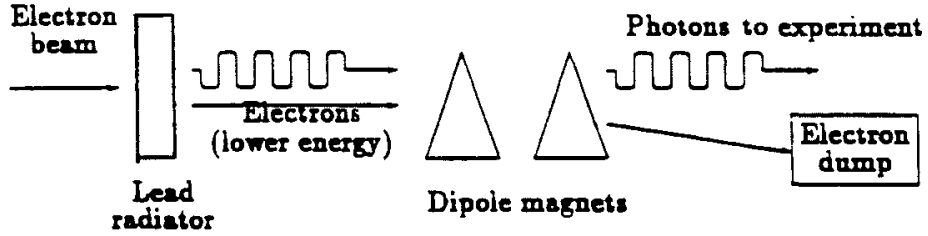

Figure 2.3: Photon Beam

Then, the electrons are swept into the RESH counters where their energy is measured $\left(E_{R E S H}\right)$, while the photons with energy

$$
E_{\gamma}=E_{0}-E_{R E S H}
$$

goes directly to the experimental target, in which the events are produced and detected in the spectrometer which we describe in the next section.

It is important to note the flexibility of the Wide Band line [23], for instance, by removing the bend (turning off the magnets) around the neutral dump we obtained a single muon beam that we used to calibrate the spectrometer, in particular, the muon system studied in the next chapter.

\subsection{Spectrometer}

In addition to the experimental target, the spectrometer (figure 2.4) is composed of four basic sections: the tracking, particle identification, beam monitoring and trigger/veto counters. 


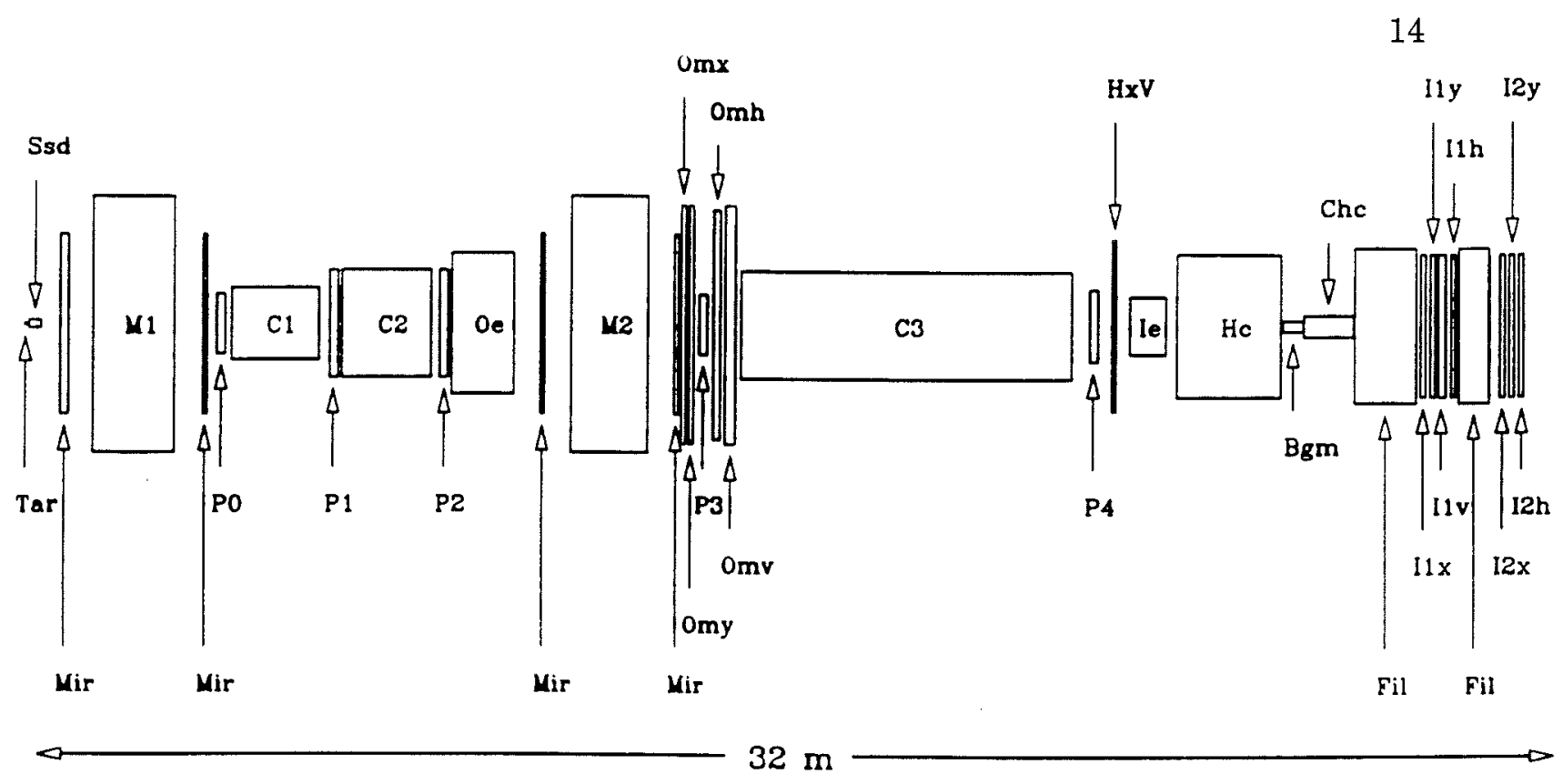

Figure 2.4: Spectrometer Side View

The first section of the detector is composed of two magnets (M1 and M2), a multiwire proportional chamber system (PWC) and a microstrip detector (SSD). Both magnets are identical in material (iron), size (width $3.5 \mathrm{~m}$, height $5.46 \mathrm{~m}$ and length $2.54 \mathrm{~m}$ ), aperture $(0.76 \mathrm{~m}$ by $1.27 \mathrm{~m}$ ) and both deflect the particles in the vertical plane but in different ways: $M 1$ deflects negative particles down while M2 deflects them up. M1 is located $2.4 \mathrm{~m}$ downstream of the target, giving an acceptance of $\pm 100 \mathrm{mr}$ in the horizontal (no bend) plane and $\pm 170 \mathrm{mr}$ in the vertical (bend) plane. M2 is located at $12.4 \mathrm{~m}$ from the target. Its acceptance is $\pm 27 \mathrm{mr}$ in the non bending plane and $\pm 47 \mathrm{mr}$ in the bending plane. Shields of steel (plates) have been installed upstream and downstream of both magnets to stop the magnetic field from going too far outside of the aperture. The magnetic field in M1 is selected in such a way that low energy $e^{-} e^{+}$pairs are swept out and do not reach the PWC's downstream of M1. It is done setting the current in M1(M2) at 1020(2000) $A m p$ given a magnetic field in the central region of $\mathrm{M} 1(\mathrm{M} 2)$ an average value of 6.6 (12.7) Kgauss. These number give a momentum kick of $0.39 \mathrm{GeV}$ in $\mathrm{M} 1$ and $-0.834 \mathrm{GeV}$ in M2.

The reconstruction of the trajectory of charged particles downstream of $\mathrm{M} 1$ is done by the PWC system. The PWC system consists of 5 stations of chambers (P0 to P4). P3 and P4 are located downstream of M2 and P0, $\mathrm{P} 1$ and $\mathrm{P} 2$ are placed between the magnets. Each station of chamber is filled with a mixture of gas (65\%-35\% of Argon and Ethane at $1 \mathrm{~atm})$ and the 
Table 2.1: Multiwire Chamber Setup

\begin{tabular}{|c|l|c|c|r|c|}
\hline $\begin{array}{c}\text { Chamber } \\
\text { Name }\end{array}$ & $\begin{array}{l}\text { Planes } \\
\text { Type }\end{array}$ & $\begin{array}{c}\text { Number } \\
\text { of Wires }\end{array}$ & $\begin{array}{c}\text { Spacing } \\
\mathrm{mm}\end{array}$ & $\begin{array}{c}\text { Plane Size } \\
\mathrm{cm}^{2}\end{array}$ & $\begin{array}{c}\text { Z Position } \\
\mathrm{cm}\end{array}$ \\
\hline P0 & $x u v y$ & 2284 & 2 & $76.2 \times 127.0$ & 398 \\
P1 & $x u v y$ & 2932 & 3 & $152.4 \times 228.6$ & 635 \\
P2 & $x u v y$ & 2900 & 3 & $152.4 \times 228.6$ & 869 \\
P3 & $x u v y$ & 2260 & 2 & $76.2 \times 127.0$ & 1440 \\
P4 & $u v$ & 1869 & 2 & $101.6 \times 152.4$ & 2284 \\
& $x$ & & 3 & & \\
\hline
\end{tabular}

typical working voltage is $3000-3500 \mathrm{~V}$. They are composed of proportional wire planes (namely $x, u, v$ and $y$ ) which provide the coding necessary to determine unambiguously the position of the tracks at this location. Wires in the $v$-plane are at an angle whose tangent is $-1 / 5$ with respect to the horizontal plane $(y)$. The tangent of the angle of the $u$-plane is $1 / 5$ relative to the $y$-plane. The $x$ plane measures non-bend view horizontal coordinate. The number of planes, number of channels, spacing of the wires, active area and the $Z$ position with respect to the target for each chamber are shown in table 2.1.

The resolution in the determination of the momentum of the track using M2 is

$$
\frac{\Delta P}{P}=1.4 \%\left(\frac{P}{100 G e V}\right) \sqrt{1+\left(\frac{23 G e V}{P}\right)^{2}}
$$

and the momentum resolution in $\mathrm{M} 1$ is

$$
\frac{\Delta P}{P}=3.4 \%\left(\frac{P}{100 G e V}\right) \sqrt{1+\left(\frac{17 G e V}{P}\right)^{2}}
$$

where the second term in the square root represents the multiple scattering error due to the material between the magnets.

Upstream of M1, the reconstruction of the trajectory of charged particles is carried out by the silicon microstrip detector SSD located between the target and M1. The SSD consists of $12 S i$ microstrip planes (8256 channels) grouped in four stacks of three planes each $(x, y, u)$ oriented at $225^{\circ}, 315^{\circ}$, and $270^{\circ}$ with respect to $x$ axis of the reference system (figure 2.5). The active area of the detector is $25 \times 35 \mathrm{~mm}^{2}$ for the first stack and $50 \times 50 \mathrm{~mm}^{2}$ for the others [24]. Each stack has a high resolution central region. In the central region, the first stack is $10 \mathrm{~mm}$ wide and has a pitch of $25 \mu \mathrm{m}$, the lateral region is $7.5 \mathrm{~mm}$ wide and has a $50 \mu \mathrm{m}$ pitch. All the dimensions and pitches 


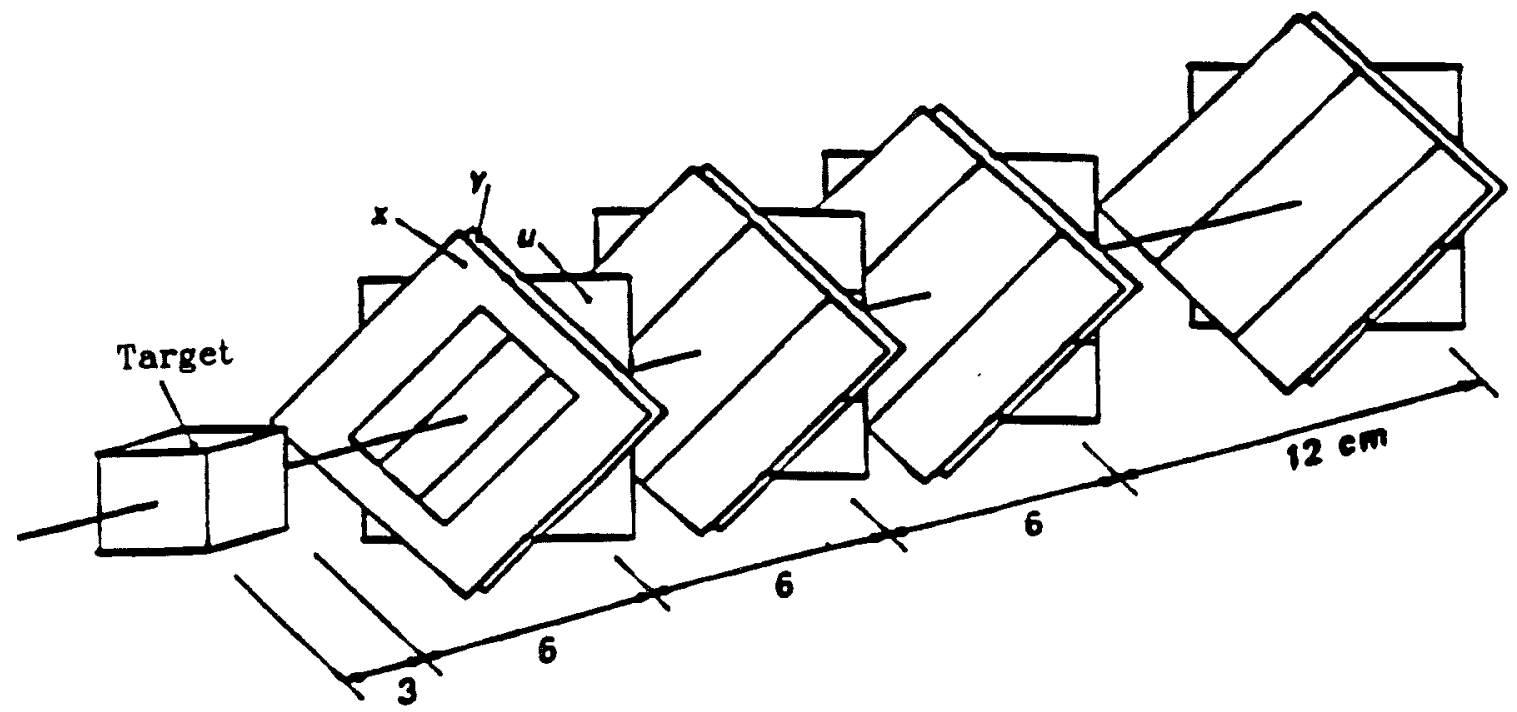

Figure 2.5: SSD Detector

of the remaining stacks are double. The detectors are stacked $5 \mathrm{~mm}$ away from each other in each group and they have $300 \mu \mathrm{m}$ thickness.

Two important characteristics of the SSD or microvertex detector are the high resolution and the high read out rate [25]. Because of the high resolution in the central region

$$
\begin{gathered}
\sigma_{x}=11.0 \mu m \sqrt{1+\left(\frac{17.5 \mathrm{GeV}}{P}\right)^{2}} \\
\sigma_{y}=7.7 \mu m \sqrt{1+\left(\frac{25.0 \mathrm{GeV}}{P}\right)^{2}}
\end{gathered}
$$

we can reconstruct the decay vertex of the event with high precision. Since the gate of charge integration of the electronics associated to the SSD is $200 \mathrm{nsec}$, it allows to tolerate a very high rate of interactions.

The second section of the spectrometer consists of the particle identification detectors. These are the cerenkov system, muon system and calorimeters and are designed for identifying $K^{ \pm}, \pi^{ \pm}, p, \mu, n, e, K^{0}, \pi^{0}, \gamma$, etc. The identification of $\mu$ 's is performed by the muon system which is composed of scintillator and proportional tube planes. The description of the muon system detector is presented in the next chapter.

The identification of the charged particles $(\pi, K$, and $p)$ is done by three cerenkov counters, C1, C2 and C3 (figure 2.4). The main characteristics of each counter (gas mixture,number of cells, transverse dimension and depth) are shown in table 2.2 where we have included the momentum threshold for $\pi, K$, and $p[22]$. 
Table 2.2: Cerenkov Counters

\begin{tabular}{|c|c|c|c|c|c|c|c|}
\hline & Gas & Cells & $\begin{array}{c}\text { Dimension } \\
\mathrm{cm}^{2}\end{array}$ & $\begin{array}{c}\text { Depth } \\
\mathrm{cm}\end{array}$ & $\begin{array}{c}\pi \\
\mathrm{GeV} / \mathrm{c}\end{array}$ & $\begin{array}{c}K \\
\mathrm{GeV} / \mathrm{c}\end{array}$ & $\begin{array}{c}p \\
\mathrm{GeV} / \mathrm{c}\end{array}$ \\
\hline $\mathrm{C} 1$ & $\mathrm{HeN}_{2}$ & 90 & $122.1 \times 205.3$ & 182.1 & 6.6 & 23.2 & 44.3 \\
$\mathrm{C} 2$ & $\mathrm{~N}_{2} \mathrm{O}$ & 110 & $152.4 \times 228.6$ & 181.8 & 4.5 & 16.1 & 30.9 \\
$\mathrm{C} 3$ & $\mathrm{He}$ & 100 & $152.4 \times 228.6$ & 662.9 & 17.2 & 61.0 & 116.2 \\
\hline
\end{tabular}

This system is designed in such a way that allows the distinction of particles in different ranges of momentum. For hadron identification we can observe different ranges of momentum. For example, the best discrimination range of momentum is between 16.1 and $44.3 \mathrm{GeV} / \mathrm{c}$ where we can distinguish definitively $\pi, K$ and $p$. Between 4.5 and 16.1 or 44.3 and $61 \mathrm{GeV} / c$ we can separate $\pi$ from $K / p$ ambiguity. For 61 to $116.2 \mathrm{GeV} / \mathrm{c}$ we separated $p$ from the ambiguity $\pi / K$. Also is important to note that the cerenkov system can be used to identify low momentum electrons because, with the exception of $\mu$ 's which are relatively rare, they are the only particles which produce light below $\pi$ threshold. This electron identification at low momentum can be complemented with the electron identification at higher momentum in the calorimeters.

The identification and the measurements of the energy for $e^{\prime} \mathrm{s}, \pi^{0}$ 's, and $\gamma$ 's is done by the outer (OE) and inner (IE) electromagnetic calorimeters. The main characteristics of $\mathrm{OE}$ and IE are described in [27]. OE(IE) is a lead-aluminium-scintillator sandwich shower detector located upstream (downstream) of M2 and is divided in 4(3) blocks, OE0, OE9, OE1 and OE2 (IE1, IE2 and IE3) along the beam direction as shown in figure 2.6. The transverse dimension of OE1(IE1) is 2.7 (1.12) $m$ in $x$ and 2.5 (1.12) $m$ in $y$. The thickness of the detectors are shown in figure 2.6. The central region of $\mathrm{OE}(\mathrm{IE})$ is a hole of about $88 \times 51(10.2 \times 10.2) \mathrm{cm}^{2}$ letting the non interacted photon in the target, high momentum particles at small angle or the electron pair pass through the detector without striking the $\mathrm{OE}(\mathrm{IE})$. The energy resolution of $\mathrm{OE}(\mathrm{IE})$ for $\gamma$ or $e$ in energy less than $20 \mathrm{GeV}$ (up to $100 \mathrm{GeV}$ ) region is $(\Delta E / E)_{F W H M}= \pm 0.23 / \sqrt{E}$.

The identification of the neutral hadrons is performed by the hadron calorimeter ( $\mathrm{HC}$ ) shown in figure 2.7. It is made by 28 layers of $\mathrm{Fe}$ alternating with 28 planes of detectors. Each plane of detector consists of Iarocci tubes which contain a mixture of gas (50\%-50\% Argon and Ethane). The anode is a wire of Tungsten with golden plating. The pad read-out signal has a radial symmetry so that the transverse energy can be promptly available. The 


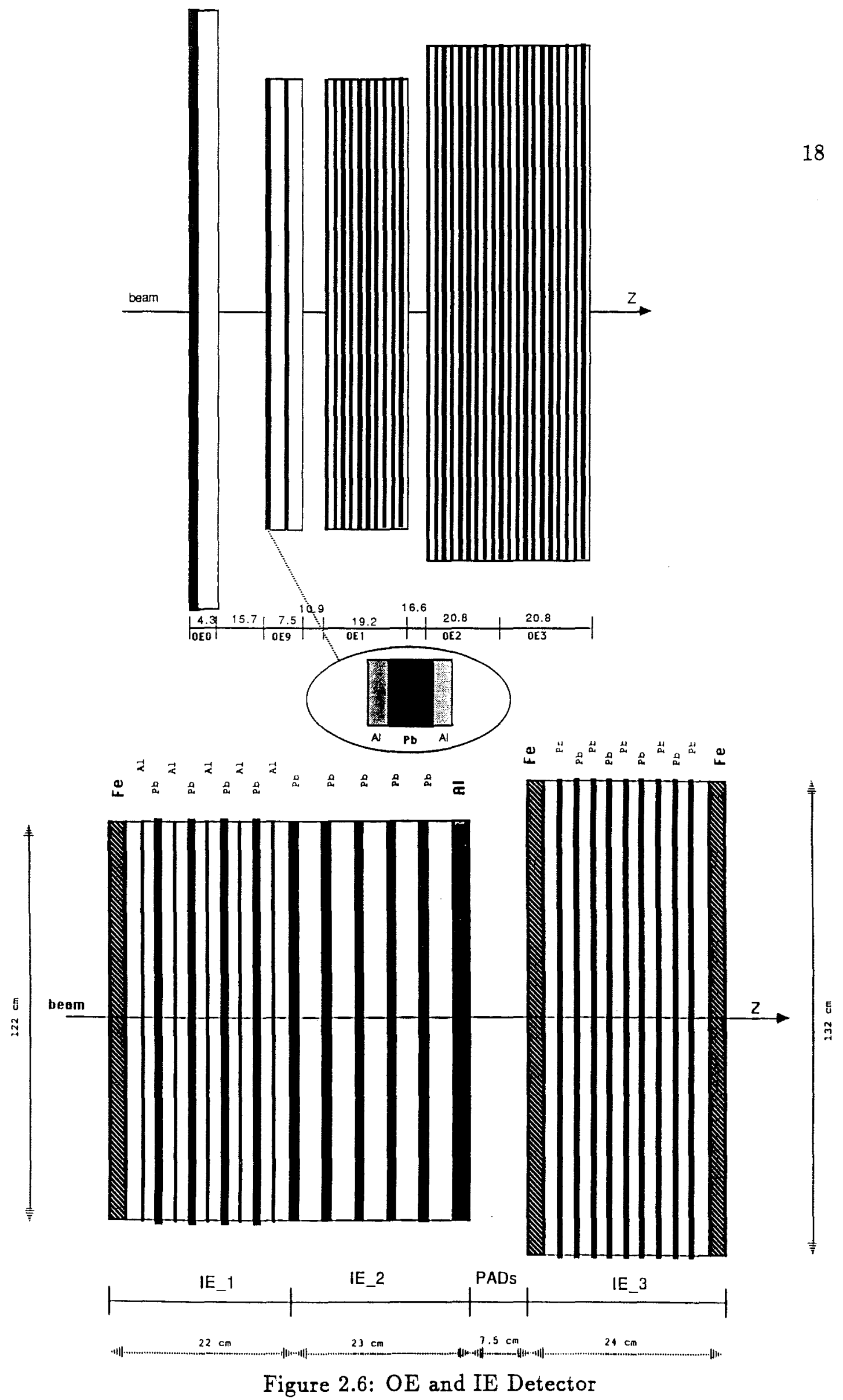




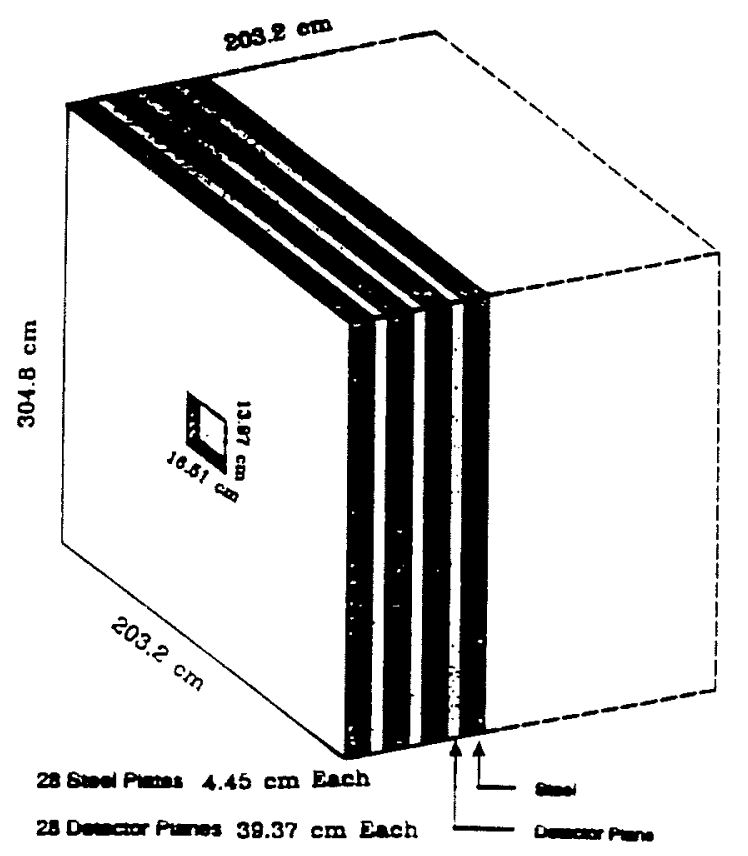

Figure 2.7: HC Detector

resolution in energy of $\mathrm{HC}$ is given by $\Delta E / E \sim(0.7 \rightarrow 1) / \sqrt{E}$. The central region of $\mathrm{HC}$ is a hole whose dimension match with the one in IE to allow that $\gamma$ or $e^{-} e^{+}$pair goes directly to the beam gamma monitor (BGM) counter .

The third section (beam monitoring) of the spectrometer is design to measure the energy and intensity of the incident beam. It is composed by central hadron calorimeter ( $\mathrm{CHC}), \mathrm{BGM}$ and the recoil electron shower (RESH) detector.

$\mathrm{BGM}$ and $\mathrm{CHC}$ are located between $\mathrm{HC}$ and the muon system (figure 2.4). They cover the central region of the spectrometer. The transverse area of BGM is $20.32 \times 20.32 \mathrm{~cm}^{2}$ and $60 \mathrm{~cm}$ of thickness. It covers an angular region of $\pm 4 \mathrm{mr}$ and is composed by 45 layers of $\mathrm{Pb}$ (thickness $0.32 \mathrm{~cm}$ ) with Lucite (thickness $1 \mathrm{~cm}$ ). The light coming from the Lucite is directed to only one phototube. Just behind of BGM is located CHC. It is composed of 16 layers of Uranium of total thickness of $60.96 \mathrm{~cm}$. To protect and reduce the counter noise due the natural radioactivity of the Uranium, each layer is put inside of a container of $F e$. The layers are alternate with 16 scintillator strips which are read-out by two phototubes. The global energy resolution (for incident hadrons) of the system $\mathrm{BGM}$ and $\mathrm{CHC}$ is $\Delta E / E \sim 6 \%+100 \% / \sqrt{E}$.

Other properties (total radiation length, energy loss and multiple scattering) of $\mathrm{OE}, \mathrm{IE}, \mathrm{OE}, \mathrm{CHC}$ and $\mathrm{BGM}$ are described in the next chapter.

We received $\sim 1.8 \times 10^{12}$ protons $/$ spill in the proton east beam line

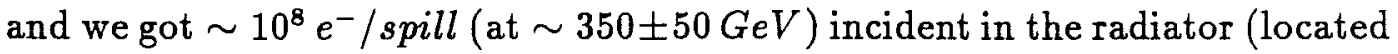
in the wide band) to produce by bremsstrahlung a photon beam and recoil electron. The shape of the photon spectrum is $\sim 1 / k$ and $\sim 10^{7} \gamma /$ spill are 
Table 2.3: Target Setup

\begin{tabular}{|c|c|c|}
\hline Target & $\begin{array}{c}\text { Thickness } \\
c m\end{array}$ & $\begin{array}{c}\text { Transverse Size } \\
c m\end{array}$ \\
\hline Be 1 & 0.8128 & $2.54 \times 2.54$ \\
Air Gap & 0.0200 & \\
Be 2 & 0.8128 & $2.54 \times 2.54$ \\
Air Gap & 0.1600 & \\
Be 3 & 0.8128 & $1.00 \times 1.00$ \\
Air Gap & 0.0800 & \\
Be 4 & 0.8128 & $1.00 \times 1.00$ \\
Air Gap & 0.0800 & \\
Be 5 & 0.8128 & $1.00 \times 1.00$ \\
\hline
\end{tabular}

incident in the experimental target. A big fraction of these photons interacted in the target producing $\sim 5 \times 10^{5} e^{-} e^{+}$pair per spill and only $\sim 3000$ hadronic interaction (wanted events). These particles $e^{-} e^{+}$and uninteracting $\gamma$ 's are directed to BGM and $\mathrm{CHC}$ to count them and to measure their energy. The measurement of the recoil electron energy is done by the RESH counter. It is a calorimeter of lead-scintillator sandwich and is located upstream of the target. Once we measure the energy of the recoil electron $\left(E_{R E S H}\right)$ and the non interacting photon energy $\left(E_{B G M}\right)$, using equation 2.1 , we calculated the interacted photon energy,

$$
E_{\gamma_{I n t}}=350 \pm 50-E_{R E S H}-E_{B G M}[G e V] .
$$

Three different targets had been used in the experiment; the silicon active target [28], scintillating fiber target [29] and Be. We used two configurations of Be target, one is composed of 4 blocks and the other one with 5 blocks of Be separated by a small gap of air. Data which comes from 5 pieces of Be target (figure 2.8) was studied in this thesis. The dimensions of the target are shown in table 2.3.

The last section of the spectrometer is the veto and trigger counters composed by several scintillators counters located upstream and downstream of the target.

\subsection{Trigger}

The trigger of the experiment is divided in two levels. The purpose of the first level trigger or master gate is to reject the electromagnetic interaction 


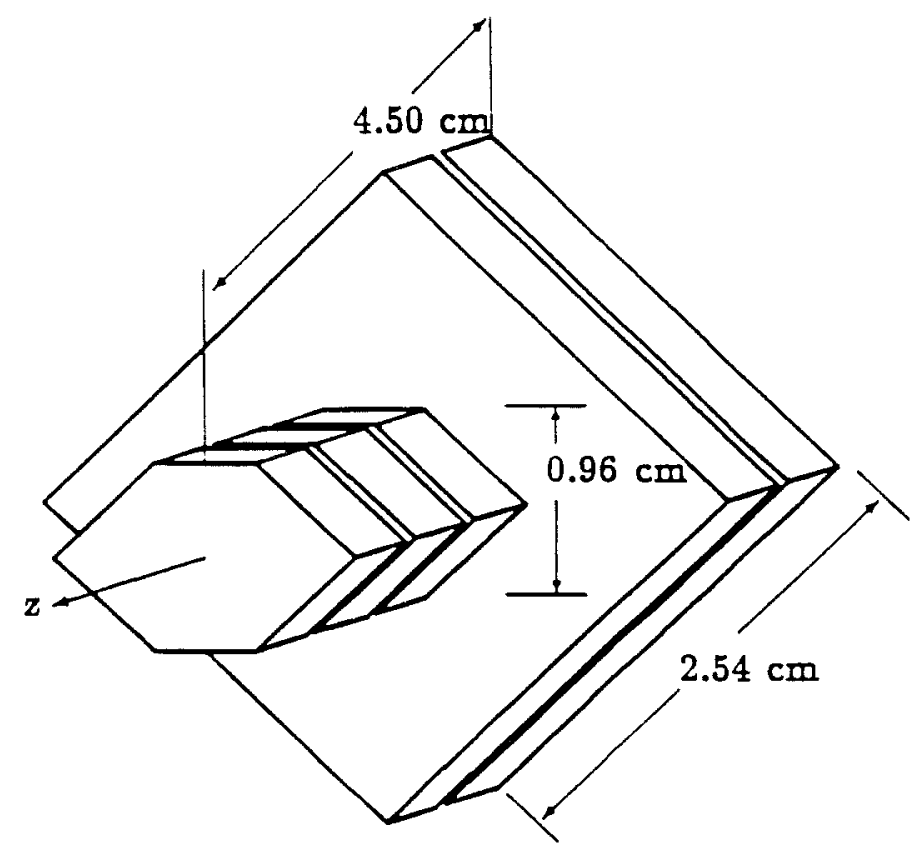

Figure 2.8: Be Target

( $e^{-} e^{+}$pair) and the second level is to select events with a minimum amount of hadronic energy or at least 2 muons.

The master gate is made by a set of scintillators counters (A0, AM, TM, TR, OH, and $H \times V$ ) placed transversally to the beam. The first 3 set of scintillators counters ( $A M, T M$ and $A 0)$ are located upstream of the target and they are designed to detect and reject charged particle travelling inside and close to the beam. AM(TM) is a set of $24(2)$ counters of dimension of about $1.5 \times 2.5 \mathrm{~m}^{2}$ situated at $\sim 300(50) \mathrm{cm}$ from the target. They are surrounding the beam line. A0 is only one small counter located at $\sim 100 \mathrm{~cm}$ and is placed inside of the beam line.

The second 3 set of counters (TR, OH and $H \times V$ ) are located downstream of the target. TR is composed of 2 small counters, TR1 and TR2 placed upstream and downstream of the SSD respectively. TR1, coincident with TR2 (TR1ৎTR2), indicates that an interaction occurred in the target. $\mathrm{OH}$ is one plane of 24 scintillator counters covering the upstream face of $\mathrm{OE}$ and is designed to detect a charge particle with big opening angle (figure 2.9). The center of this plane contains an aperture of similar dimension as OE. Finally behind the last chamber (P4) we have $H \times V$ which are 2 planes of scintillators with 24 and 12 counters placed horizontally $(H)$ and vertically (V) respectively. Each plane is composed of 2 panels positioned side by side with a separation of $\sim 7.62 \mathrm{~cm}$ in between (figure 2.9). The configuration of the magnetic field is such that the $e^{-} e^{+}$pair is deflected and directed to this 

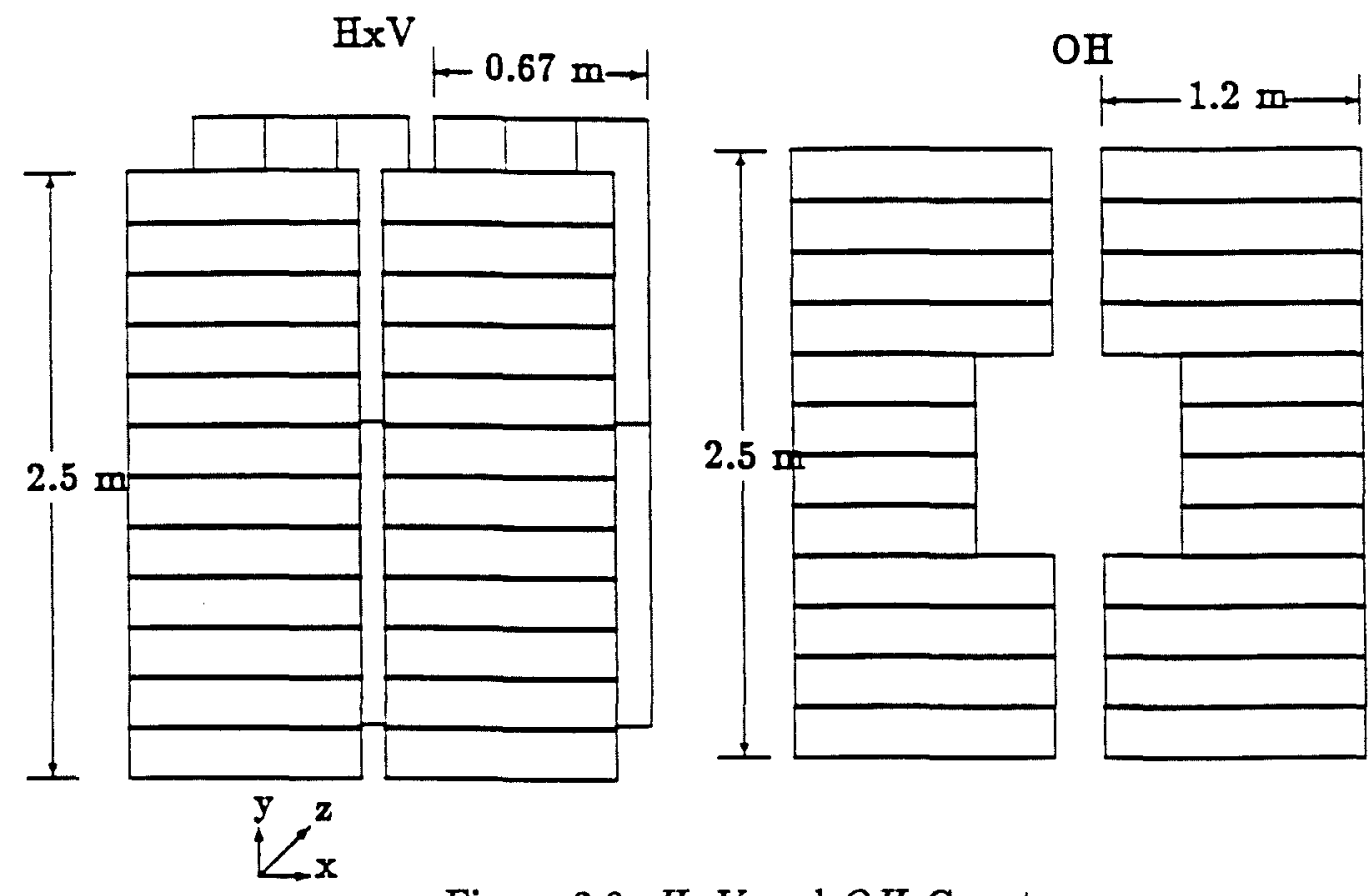

Figure 2.9: $H \times V$ and $O H$ Counters

gap and then the event is rejected from our raw data.

The master gate logic is then made up by combinations of coincidences and anticoincidences between these counters. In addition to rejecting pairs, the first level trigger is design to reject a single charged particle events, either from the incident beam or from a missing hadronic event. It is done by requiring the following master gate $\left(T_{1}\right)$,

$$
T_{1}=(\overline{A 0} \odot \overline{A M}) \odot\left(T R_{1} \odot T R_{2}\right) \odot\left[(H \times V)_{2} \oplus O H \odot(H \times V)_{1}\right],
$$

where $(H \times V)_{i=1,2}$ is the $i$ body $H \times V$ trigger. $(H \times V)_{1}$ is satisfied if we have at least one cross coincidence between 2 counters on, one in $H$ and one in V. Essentially $(H \times V)_{2}$ is satisfied when we have at least $(H \times V)_{1}$ in each side of $H \times V$. The $O H \cdot(H \times V)_{1}$ trigger requires at least one particle crossing all the way (track) through the spectrometer $\left((H \times V)_{1}\right)$ and one additional track (stub) reaching M2 but hitting its yoke. However, $(H \times V)_{2}$ require at least 2 tracks. Both combinations are complemented by the requirement of detect interaction

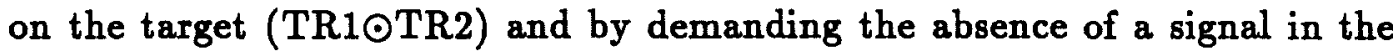
counters upstream of the target (veto).

The next selection of the event is passed through the second level trigger,

$$
T_{2}=H C \oplus(I M \odot I M) \oplus(I M \odot O M) .
$$

$T_{2}$ require at least $30 \mathrm{GeV}$ in the hadron calorimeter or at least 2 muons. The inner muon trigger (IM) required at least one counter on in 3 out of 4 inner 
muon scintillator planes (see next chapter). The outer muon trigger (OM) required at least one counter on in 1 out of 2 outer muon scintillator planes.

Once the triggers are satisfied, the events $(\sim 3000$ events/spill) are store in 5 Fastbus memories and then sent to a magnetic tape during the interspill (40 sec) period. During the spill a fraction of events are recorded with two other pre scaled triggers, the minimum bias and the pair trigger. The minimum bias events $(\sim 100$ events/spill) are recorded without required the second level trigger to study the systematic error introduced in it. The pair

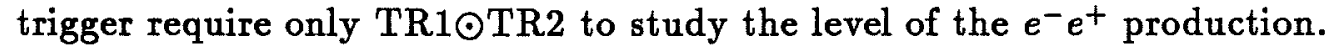




\section{Chapter 3}

\section{Muon System Calibration}

\subsection{Introduction}

The study of Charm and Bottom Photoproduction is the goal of this experiment. Many final states resulting from the decay of charm and bottom particles contain leptons, in particular of muons. The establishment of a muon identification criterion based on the study of our muon detector is therefore necessary. In order to accomplish this goal, one must have a good understanding of the efficiency and geometry of the muon system.

There are two muon systems in the spectrometer. The outer muon system (OMS) detector located downstream of the central analysis magnet (M2) is designed to detect muons at wide angles and with low momentum. The inner muon system (IMS) located at the downstream end of the spectrometer is designed to detect muons at high momentum.

In this chapter we present our measurement of the alignment of the muons detector with respect to the reference frame (Center of M2) and their efficiencies. Also we have measured and estimated the contribution of the multiple scattering and the energy loss due to the material upstream of these detectors: the Inner Electromagnetic Calorimeter (IE), the Hadron Calorimeter (HC) and the Hadron Absorber (Muon Steel) in the case of the IMS; the Outer Electromagnetic Calorimeter (OE) and the Iron of the M2 (Yoke) in the case of the OMS. The calculation of the multiple scattering radius and the energy loss on each plane requires the evaluation of an integral over the distribution of the material along the trajectory upstream of each plane. This can best be done by Monte Carlo and the results were incorporated in a lookup table.

In the case of the OMS, due to the magnetic field in the yoke of the magnet, the effect on the trajectory of the particle was studied. 
Our main method was to project and match charged tracks to the muon system, and because the size of our trigger counters $(\mathrm{HxV})$ is smaller than the muon planes, we do not have large statistics in the channels outside of these counters. The central region of the system is the only illuminated area. Thus, we assumed that the separation of wires on proportional tubes are the same for all the planes and there is no separation between two counters on the scintillators plane.

Finally we studied the dimuon sample and we determined a muon definition criterion that we used for the analysis of the muon trigger data taken.

\subsection{Muon System Setup}

As we mentioned in the previous chapter, one of the modes of the beam line operation is muon beam. This beam mode was use to calibrate the muon detector; a single muon traversing the spectrometer leaving a signal in each detector that the particle crossed. From our data acquisition system (DAQ), we know for each detector the total number of channels that were on and their address. We used the tracks defined by the proportional wire chamber (PWC) system to reconstruct the trajectory of the muon and the momentum if the analysis magnet was on. The tracks were classified into two groups (Tracks and Stubs) for analysis and calibration purposes. We refer to a particle as a "track" when it passes through all 5 PWC (from P0 to P4) planes, otherwise, if it passes through only 3 PWC (from P0 to P2) planes, we call it a "stub". We expected tracks to project into the IMS because it is located downstream of the experiment and stub to project into the OMS located just behind of M2 (figure 3.1).

Each muon system is composed of scintillators and proportional tube planes. IMS contain 3 scintillator counter planes and 4 proportional tube planes. OMS contain 2 scintillator counter planes and 2 proportional tube planes. The channels in the plane are placed vertically or horizontally. Planes with vertical channels are labelled with $\mathrm{V}$ for scintillator plane and $\mathrm{X}$ for proportional tube planes. Planes with channels placed horizontally are named with $\mathrm{H}$ or $\mathrm{Y}$ depending on the type of the detector. Additional labels come from the outer or inner detector; i.e. OM or IM (figure 3.2).

The geometrical characteristics and positions of the planes with respect to M2 are shown in table 3.1, where the channel size represents the horizontal size by the vertical size of each counter in the case of scintillator and the diameters of the tube by the length in the case of proportional tubes. 


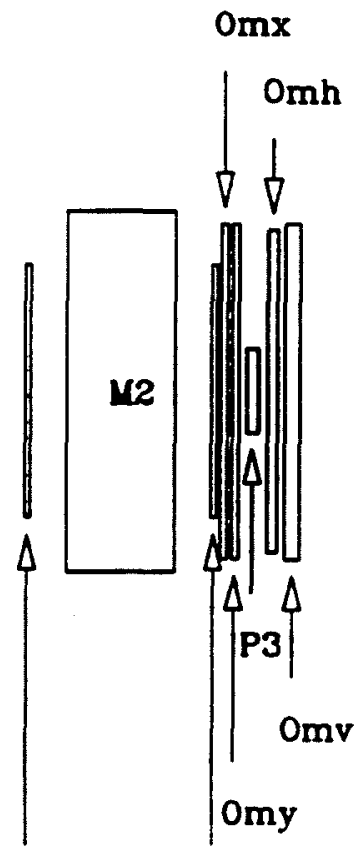

Mir

Mir

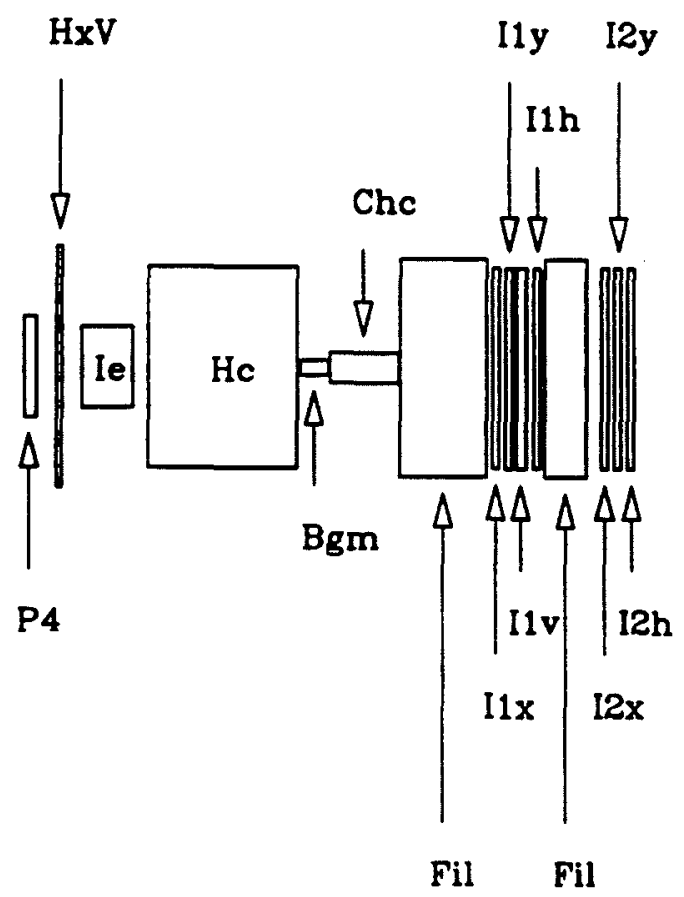

Figure 3.1: Spectrometer

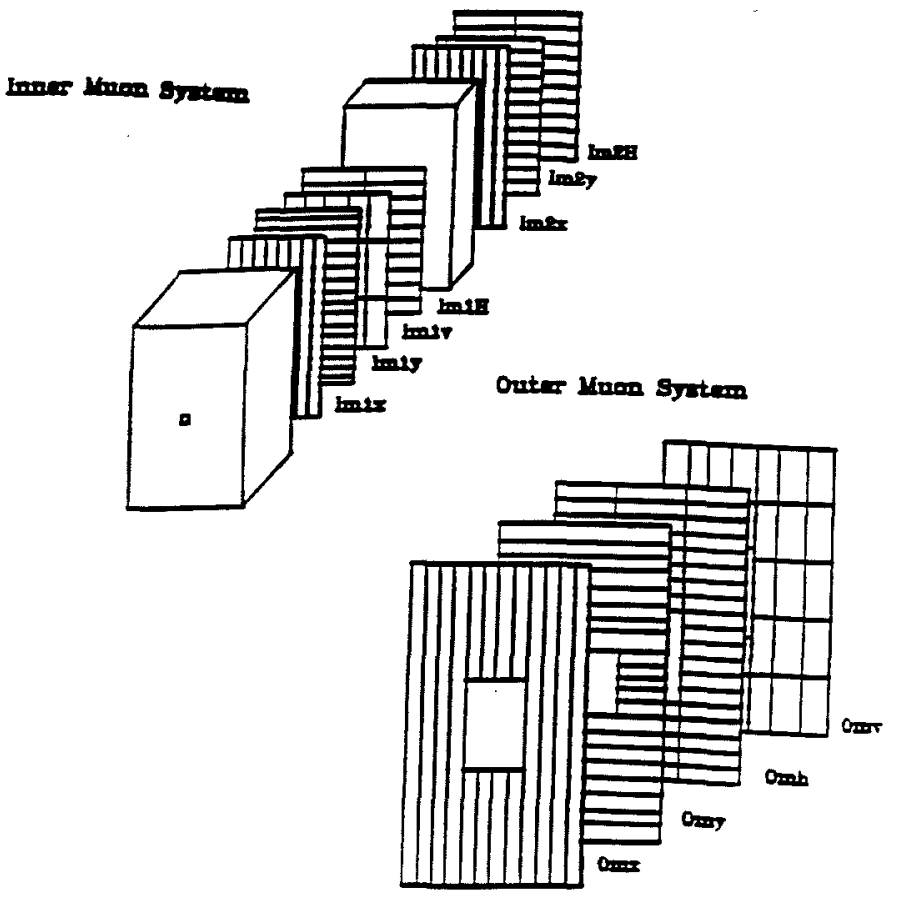

Figure 3.2: Muon System Setup 
Table 3.1: Muon System Geometry

\begin{tabular}{|l|l|c|c|r|r|}
\hline $\begin{array}{c}\text { Plane } \\
\text { Name }\end{array}$ & \multicolumn{1}{|c|}{$\begin{array}{c}\text { Detector } \\
\text { Type }\end{array}$} & $\begin{array}{c}\text { Number of } \\
\text { Channels }\end{array}$ & $\begin{array}{c}\text { Channels Size } \\
\text { Inches }\end{array}$ & $\begin{array}{c}\text { Plane Size } \\
\text { Inches }\end{array}$ & $\begin{array}{c}\text { Z Position } \\
\text { Inches }\end{array}$ \\
\hline OMX & Prop. Tube & 64 & $2 \times 200$ & & \\
OMX & Prop. Tube & 64 & $2 \times 68$ & $120 \times 200$ & 63.44 \\
OMY & Prop. Tube & 112 & $120 \times 2$ & & \\
OMY & Prop. Tube & 96 & $41 \times 2$ & $120 \times 200$ & 70.41 \\
OMH & Scintillator & 43 & $40 \times 12$ & $120 \times 192$ & 93.09 \\
OMV & Scintillator & 46 & $12 \times 40$ & $120 \times 200$ & 105.09 \\
IM1X & Prop. Tube & 64 & $2 \times 120$ & $80 \times 120$ & 683.22 \\
IM1Y & Prop. Tube & 96 & $80 \times 2$ & $80 \times 120$ & 690.98 \\
IM1V & Scintillator & 21 & $12 \times 40$ & $84 \times 120$ & 698.58 \\
IM1H & Scintillator & 20 & $40 \times 12$ & $80 \times 120$ & 707.86 \\
IM2X & Prop. Tube & 64 & $2 \times 120$ & $80 \times 120$ & 748.36 \\
IM2Y & Prop. Tube & 96 & $80 \times 2$ & $80 \times 120$ & 755.89 \\
IM2H & Scintillator & 20 & $40 \times 12$ & $80 \times 120$ & 763.83 \\
\hline
\end{tabular}

The shape of the counters and the separation of the proportional tubes are shown in figure 3.3 .

It is important to note that each plane in the OMS has a hole of dimension 40 Inches by 60 Inches located in the center of each plane. The size and the location of this aperture, which nearly coincides with the aperture of M2, is very important for muon identification purpose as will be seen in the next section.

\subsection{Counter Geometry}

As a first attempt to find the central position in $x$ and $y$ of the scintillator planes with respect to M2, we used a special muon beam run and turned off the analysis magnet of the spectrometer. Next we considered those events where we reconstructed only one track and have at least one channel on in each muon plane. This condition allows us to eliminate noisy signals by requiring the particles to reach the end of the muon detector, which tends to select muons with high momentum. This made the calculation, in some sense, independent of the material that the particle crossed as we will study in the next section. 


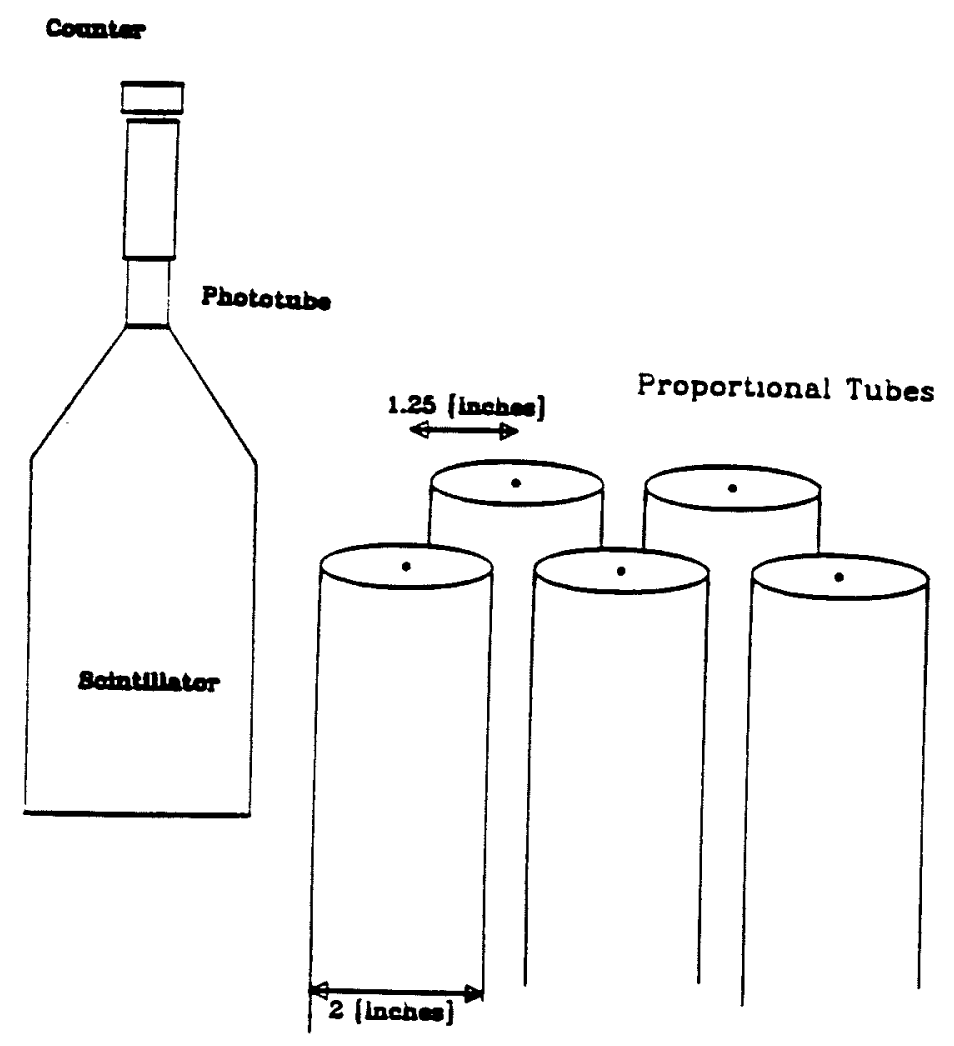

Figure 3.3: Channel Shape

Knowing the $\mathrm{Z}$ position of each plane from survey, we measured the central position of the scintillator plane using the projection of the spectrometer track. We plotted $x$ and $y$ of the projected track for the channels that were "on" to increase the statistics near of the boundaries of the channels. An example of the projection in the IMS is shown in figure 3.4.

By visual inspection of the histograms and scatter plots, we measured the position of the edges of the counter looking at the population of the projected track near to the supposed limits. The measurements of the deviation of each counter from its natural value, give us the shift of the center of the plane. The results of these measurements are shown in table 3.2.

It is important to note that the OMS values shown in table 3.2 were taken from the measurement the Alignment Group of Fermilab made because we did not have enough statistics to do the calculation. As we see in the multiplicity histograms (total numbers of hits in each plane per event) in figure 3.5, we can observe that the average multiplicity is zero, even if the stub was incident on the aperture or yoke of the magnet.

As we will see in the following sections, the use of the correct shift values improve the matching efficiency in the muon system.

Because the muon went through a large amount of material, we stud- 


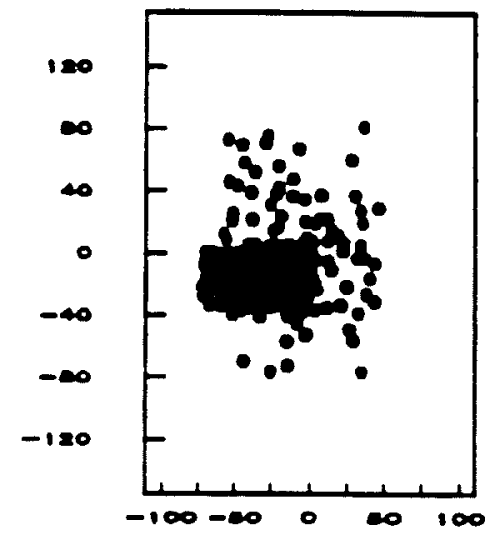

Figure 3.4: Projection

Table 3.2: Counter Planes Shift

\begin{tabular}{|l|c|c|}
\hline $\begin{array}{l}\text { Detector } \\
\text { Name }\end{array}$ & $\begin{array}{c}\text { Shift on X } \\
\text { Inches }\end{array}$ & $\begin{array}{c}\text { Shift on Y } \\
\text { Inches }\end{array}$ \\
\hline IM1V & 0.236 & -1.968 \\
IM1H & -0.397 & -0.157 \\
IM2H & -0.397 & 0.157 \\
OMH & 0.625 & 1.755 \\
OMV & 0.125 & 1.340 \\
\hline
\end{tabular}



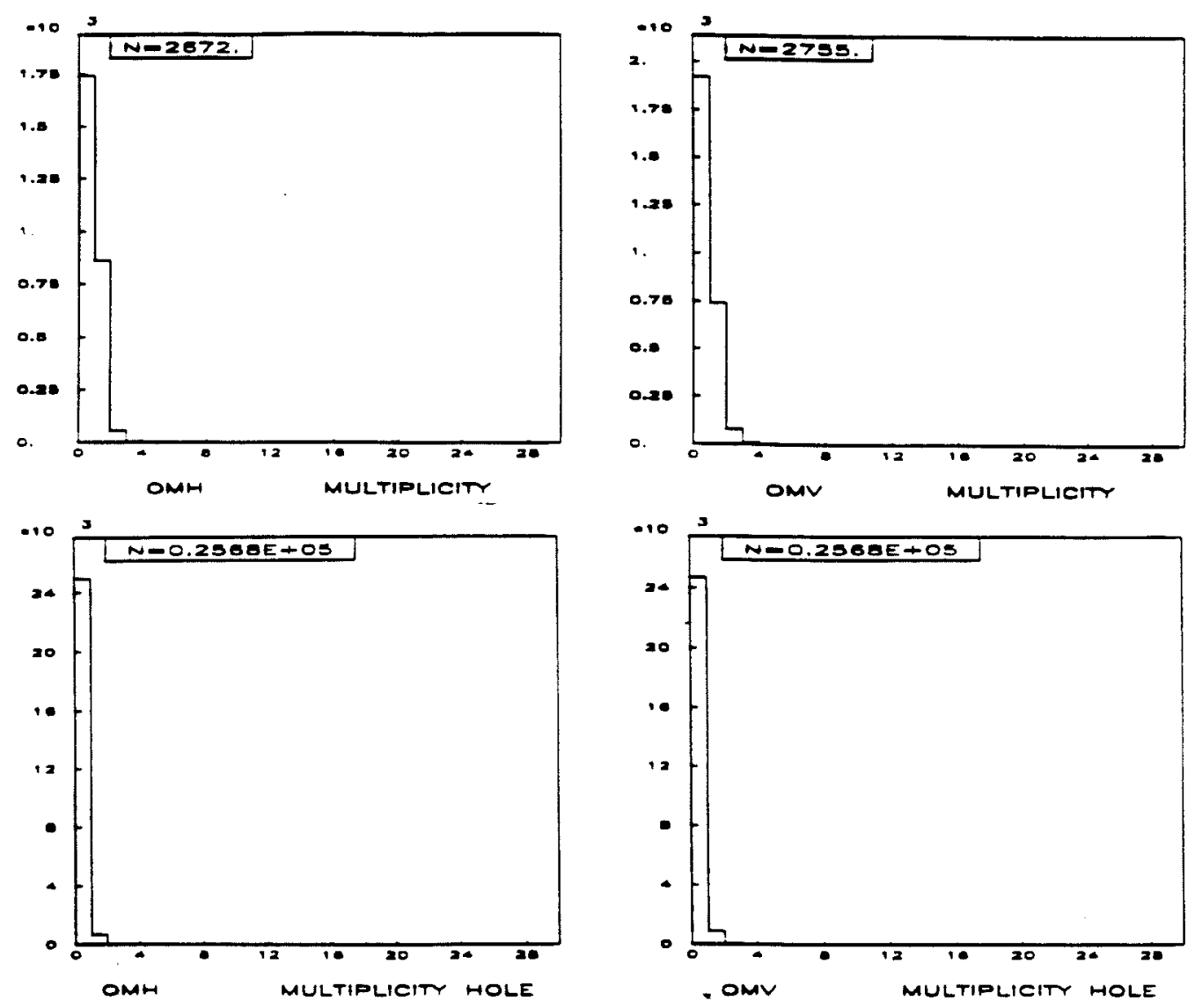

Figure 3.5: Multiplicity

ied the multiple scattering effect before measuring the efficiency of the muon system.

\subsection{Multiple Scattering Correction}

We have "some amount" of material in front of each muon system to stop hadrons. Its thickness is such that a single charged particle crossing the medium may suffer a large number of small-angle scattering collisions. It produces statistical fluctuations of the path length of the particle coming upstream of the material. These statistical phenomena are called multiple scattering and have been well described $[31,32]$.

The particle that cross the material, depending of its momentum, may change its direction suffering a small or big deviation from its original trajectory. The mean angular deflection $\theta_{m s}$ as a function of the momentum of the particle and the materials crossed, is given by the well known formula $[30]$,

$$
\theta_{m s}=\frac{0.015 \mathrm{GeV} / \mathrm{c}}{P \beta} Z_{\text {Inc }} \sqrt{\frac{L}{L_{r}}}\left[1+\frac{1}{9} \lg \left(\frac{L}{L_{r}}\right)\right] \text { Radians }
$$




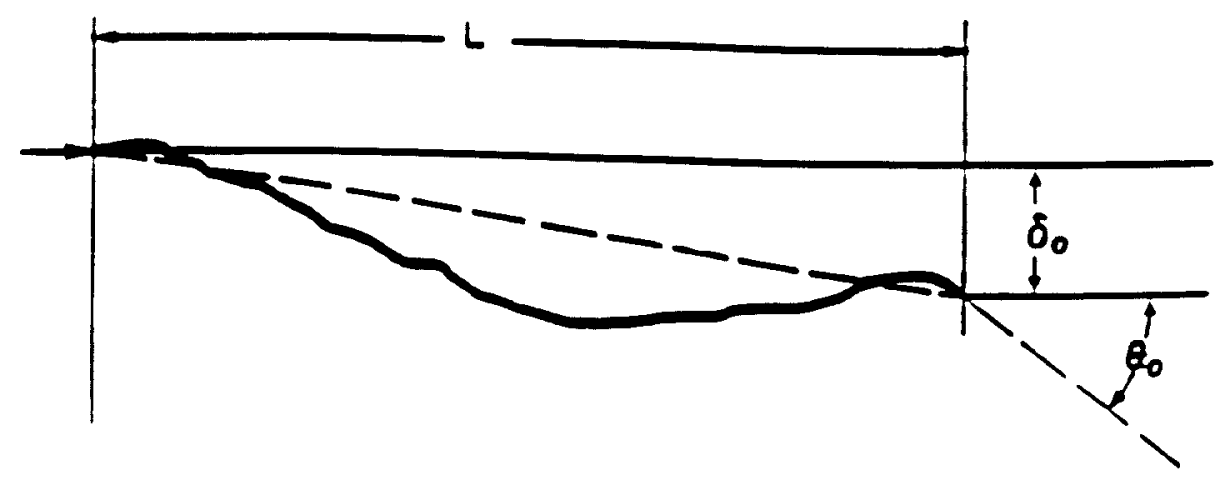

Figure 3.6: Multiple Scattering Diagram

where $\mathrm{Z}_{\text {Inc }}$ : is the charge of the particle,

$\mathrm{L} \quad$ : the length of material traversed,

$\mathrm{L}_{\boldsymbol{r}}$ : the radiation length of the material,

$\beta \quad$ : the particle velocity in units of $c$ and

$\mathrm{P} \quad$ : the incident momentum of the particle in $\mathrm{GeV} / \mathrm{c}$.

The distribution of this total deflection can be represented by a gaussian function only for small deflection angles. Moliere theory [31] corrected for large angle scattering including the effect of the Multiple Coulomb Scattering in the charged particle trajectory accounts for the non gaussian tails of the scattering distribution. The angle $\theta_{m s}$ has been calculated fitting the Moliere theory using a Gauss distribution with zero mean value and standard deviation given by $\theta_{m s}$ (equation 3.1). Under this approximation the lateral displacement $\delta_{m s}$ (figure 3.6) is:

$$
\delta_{m s}=\frac{1}{\sqrt{3}} L \theta_{m s}
$$

To evaluate the effect of the multiple scattering in the deviation of the track in our muon detector, we measured its deviation from a straight line as a function of its momentum. This deviation is the error in the projection of the track.

We take a special muon beam run with the analysis magnet on to get the momentum of the track. We projected the track in a straight line (as if there was no absorber upstream of the muon detector) on each proportional tube plane to predict the central coordinate of the wire $\left(\chi_{p}\right)$ that was hit and we looked for the coordinate of the nearest $\left(\chi_{\text {coses }}\right)$ tube "on" to the predicted one. Next we calculate the residual of the track $(\Delta \chi)$ in each plane and 

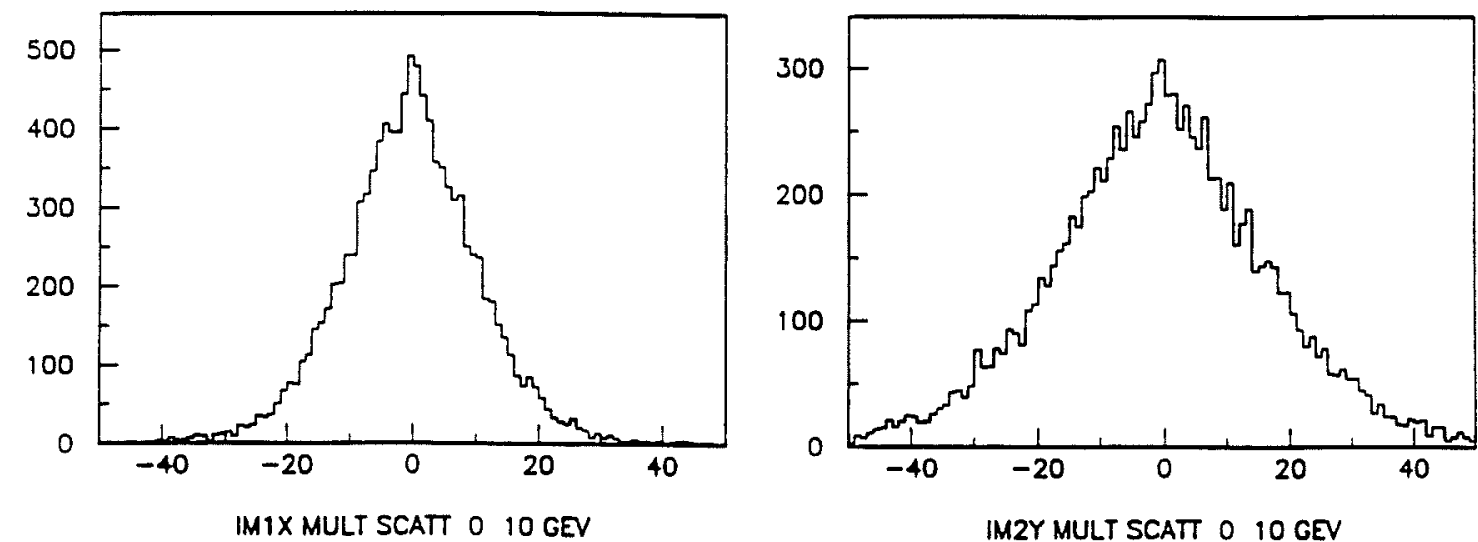

32
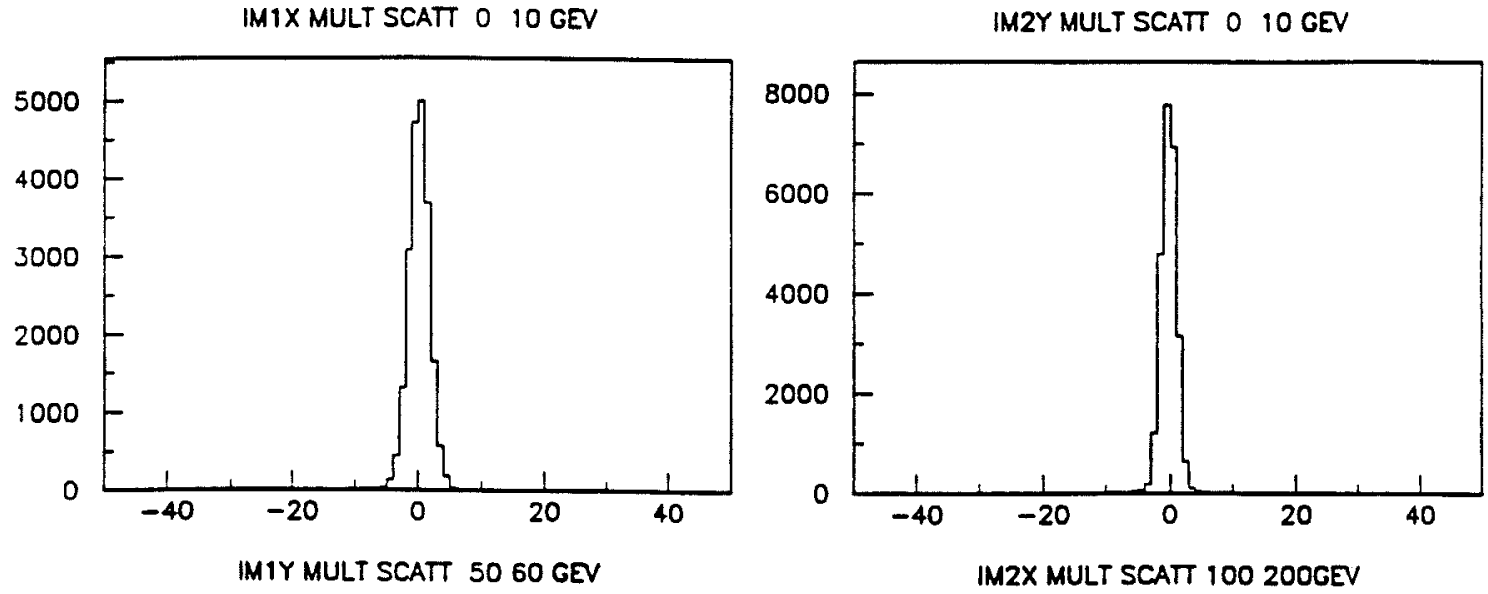

Figure 3.7: Residuals

histogrammed this quantity for incident $10 \mathrm{GeV}$ momentum intervals,

$$
\Delta \chi=\chi_{\text {close }}-\chi_{p} \text {. }
$$

These coordinates are $x$ or $y$ depending on the plane orientation. Figure 3.7 shows some of these histograms for different inner muon planes with different momentum intervals. We can observe from figure 3.7 the strong dependence of the multiple scattering on the momentum and on the material that the particle crossed. As the momentum of the particle increases, the width of the error distribution of the track becomes narrower until it reaches the expected geometric resolution of the proportional tube plane which is 0.36 Inches. We measure the standard deviation $(\sigma)$ of the residual for each average momentum (P) interval and plotted $\sigma$ versus $1 / P$ as we show in figure 3.8 for the IMS where we have included Monte Carlo data. Then to get the total error of the track $\left(\sigma_{t}\right)$ as a function of the momentum, we add the square of the uncertainties due to the multiple scattering $\left(\sigma_{m s}\right)$ and the resolution of the plane $\left(\sigma_{i}\right)$, it is;

$$
\sigma_{t}^{2}=\frac{\alpha_{i}^{2}}{P^{2}}+\sigma_{i}^{2}
$$



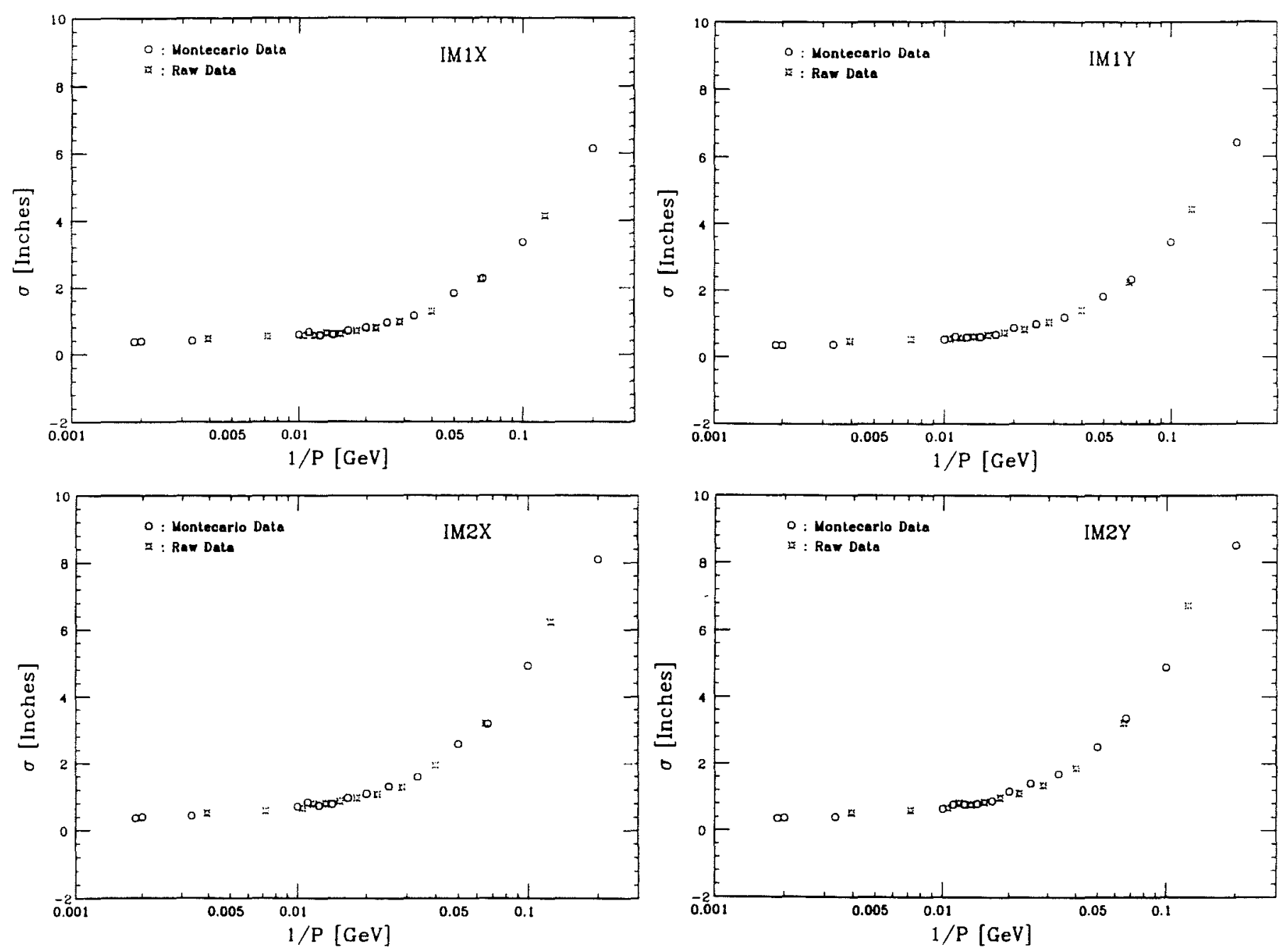

Figure 3.8: Multiple Scattering Data 
The first term of this equation represents $\sigma_{m s}^{2}$ where $\alpha_{i}$ is a constant to determine and the units are Inches - GeV.

As the multiple scattering process change the direction of the muon passing through the matter, it also losses energy $[31,34]$ changing the magnitude of its momentum ( $\mathrm{P}_{\text {Loss }}$ ). Taking this into consideration we substitute the incident momentum for the momentum of the muon when it reaches the detector,

$$
\frac{1}{P^{2}}=\frac{1}{\left(P-P_{\text {Loss }}\right)^{2}} \cong \frac{1}{P^{2}}\left(1+\frac{2 P_{\text {Loss }}}{P}\right)
$$

where we assume the correction due to the energy loss is small. By replacing equation 3.5 in 3.4 we obtain the function that we use to fit the error of the track adding the contribution of the multiple scattering, energy loss and resolution,

$$
\sigma_{t}^{2}=\frac{\alpha_{i}^{2}}{P^{2}}+\frac{\beta_{i}^{2}}{P^{3}}+\sigma_{i}^{2},
$$

where $\beta_{i}^{2}$ is strongly correlated with $\alpha_{i}^{2}$ by $\beta_{i}^{2}=2 \mathrm{P}_{\text {Loss }} \alpha_{i}^{2}$.

We used the Least Squares Fit Method [35] to find the constants $\alpha_{i}, \beta_{i}$ and $\sigma_{i}$. Due to the long tail of the multiple scattering distribution we performed two fits in each data set, one at low $\mathrm{P}$ and one at high $\mathrm{P}$. We fitted the points with $P \geq 50 \mathrm{GeV}$ to get the resolution of the planes. In this range of energy the curve is a straight line and the energy loss correction can be omitted. Once we know the value of $\sigma_{i}$, we introduced it in equation 3.6 to perform the fit at low $\mathrm{P}$, giving the values of $\alpha_{i}$ and $\beta_{i}$.

The results of the fit for each inner proportional tube plane are shown in figure 3.9 where the solid line is the result of the fit for Monte Carlo data while the dotted line is for raw data. Looking at the values $\sigma_{i}$ and $\alpha_{i}$ (where $\mathrm{i}=\mathrm{d}$ for raw data and $\mathrm{i}=0$ for Monte Carlo data) in figure 3.9 we can see there is a good agreement between our data and our simulation of the multiple scattering. This Monte Carlo muon faketape was created for different incident momentum intervals, where we have simulated the multiple scattering using Moliere theory [33].

The total material we have in front of the IMS has been included in our Monte Carlo. These detectors are; IE (Inner Electromagnetic Calorimeter with its 3 blocks, IE-1,IE-2 and IE-3), HC (Hadron Calorimeter), BGM (Beam Gamma Detector), CHC (Central Hadron Calorimeter), SB (Shield Blocks), MS1 (Muon Steel Block 1) and MS2 (Muon Steel Block 2). They are fundamentally composed of a combination of one or more of the following materials: Scintillator, Aluminum, Lead, Steel, Uranium and Concrete. We can express the total material of each detector knowing the total length, total radiation 

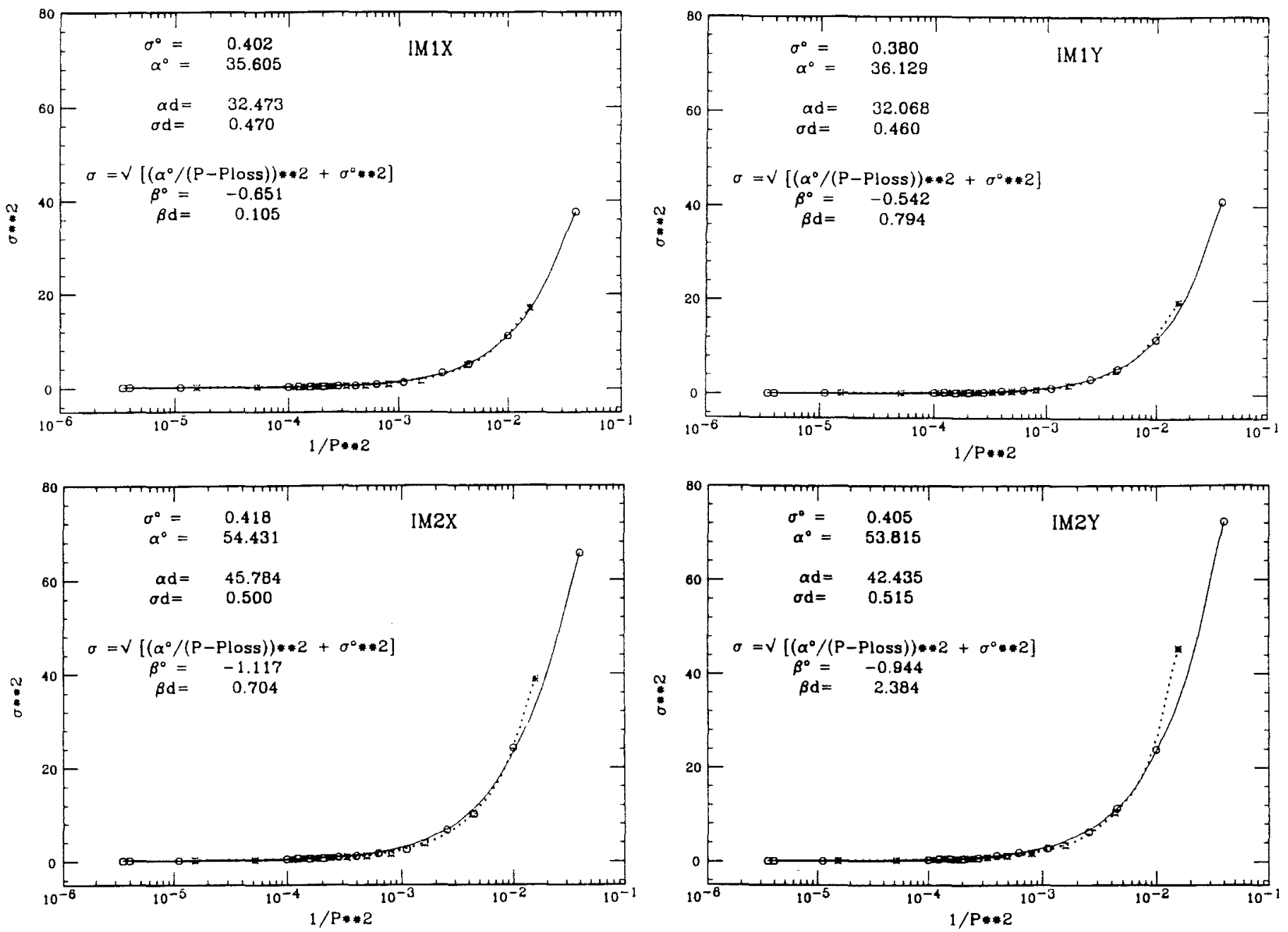

Figure 3.9: Multiple Scattering Fit 
Table 3.3: Upstream Detector of Inner System

\begin{tabular}{|l|r|c|c|}
\hline $\begin{array}{l}\text { Detector } \\
\text { Name }\end{array}$ & $\begin{array}{c}\text { Length } \\
C m\end{array}$ & $\begin{array}{c}\text { Radiation Length } \\
L_{r}\end{array}$ & $\begin{array}{c}\text { Energy Loss } \\
\text { GeV }\end{array}$ \\
\hline & & & \\
IE 1 & 22.00 & 6.566 & 0.092 \\
IE 2 & 23.00 & 6.521 & 0.072 \\
IE 3 & 24.00 & 13.210 & 0.141 \\
HC & 219.30 & 72.085 & 1.478 \\
BGM & 40.64 & 72.571 & 0.521 \\
CHC & 101.60 & 194.827 & 1.348 \\
SB & 142.24 & 13.293 & 0.605 \\
MS1 & 128.60 & 73.068 & 1.498 \\
MS2 & 63.00 & 35.795 & 0.734 \\
\hline
\end{tabular}

length and the total energy loss. These quantities are shown in table 3.3. The calculated energy loss is the summation of the material along the path;

$$
E_{\text {Loss }}=\sum_{i=\text { Material }}^{n}\left(\frac{d E}{d x}\right)_{i} \rho_{i} L_{i}
$$

where $\rho_{i}\left(\mathrm{gr} / \mathrm{cm}^{3}\right)$ is the mass density, $L_{i}(\mathrm{~cm})$ the total length of the material and $d E / d x$ the energy loss gradient $\left(M e V \mathrm{~cm}^{2} / g r\right)$.

The particle that reach the inner detector can go through different paths, essentially we can distinguish 4 cases (see table 3.4); for example, Case I: through BGM, CHC and MS1 for the first set of the inner planes (IM1X, IM1Y) and through BGM, CHC, MS1 and MS2 for the second set of the inner planes (IM2X, IM2Y).

To check the values that we got from the fit, we did a simple analytical calculation of the total radiation length $\left(\mathrm{L}_{r}\right)$, loss momentum $\left(\mathrm{P}_{\text {Loss }}\right)$ and multiple scattering parameters $(\alpha)$ based in equation 3.1. Adding all the material through the different paths of the particle we calculated the values shown in table 3.4.

Because of the size of the trigger counters, only the central region of the detectors upstream of the muon system is illuminated. There is a high probability that the track that hit the IMS had been crossing through the material as is described in case II of table 3.4. Taking case II as a reference values, we can see that the values we got for $\alpha_{i}$ from the fit values are the values that we expected. The resolution that we expected ( $\sigma=0.36$ Inches) had been reproduced in the Monte Carlo and in the raw data. Comparing the 
Table 3.4: Analytical "Inner" Calculation

\begin{tabular}{|c|c|c|c|}
\hline Path & & Inner-1 & Inner-2 \\
\hline Case I & & & \\
Bgm-Chc- & & & \\
\cline { 2 - 4 } Ms1-Ms2 & $\mathrm{L}_{r}$ & 340.46 & 376.26 \\
\cline { 2 - 4 } & $\mathrm{P}_{\text {Loss }}$ & 3.36 & 4.10 \\
\cline { 2 - 4 } & $\alpha$ & 25.91 & 34.45 \\
\hline Case II & & & \\
\cline { 2 - 4 } Ie1-Ie2-Ie3-Hc- \\
Sb-Ms1-Ms2 & $\mathrm{L}_{\boldsymbol{r}}$ & 184.74 & $\mathbf{2 2 0 . 5 3}$ \\
\cline { 2 - 4 } & $\mathrm{P}_{\text {Loss }}$ & 3.88 & 4.61 \\
\cline { 2 - 4 } & $\alpha$ & 36.18 & 45.06 \\
\hline Case III & & & \\
Ie3-Hc-Sb- & $\mathrm{L}_{r}$ & 171.65 & 207.45 \\
\cline { 2 - 4 } Ms1-Ms2 & $\mathrm{P}_{\text {Loss }}$ & 3.72 & 4.45 \\
\cline { 2 - 4 } & $\alpha$ & 32.29 & 40.86 \\
\hline Case IV & & & \\
Hc-Sb- & $\mathrm{L}_{r}$ & 158.44 & 194.24 \\
\cline { 2 - 4 } Ms1-Ms2 & $\mathrm{P}_{\text {Loss }}$ & 3.58 & 4.31 \\
\cline { 2 - 4 } & $\alpha$ & 29.56 & 37.91 \\
\hline
\end{tabular}

values of $\beta_{i}\left(\equiv \mathrm{P}_{\text {Loss }}\right)$ that we got from the fit (figure 3.9 ) with our expected values of loss momentum in case II (table 3.4) we can see that we did not get the same values. To increase the interval statistics, our bin of momentum was taken larger than the energy loss correction.

Due to the effect of the magnetic field in the trajectory of the incident stub, the evaluation of the multiple scattering in the detectors upstream of the OMS is postponed to the next section.

\subsection{Magnetic Correction}

The stubs that hit the OMS detector had been passing through a different amount of material than the track did to reach the IMS detector. In fact, the track went through the aperture of the analysis magnet (M2) while the stub crossed through the steel to hit the OMS. M2 is located just upstream of the OMS. It is made [37] of blocks of Steel (yoke) and 2 coils of 200 turns each as shown in figure 3.10. The magnetic field $(\vec{B})$ as a function of the electric current (I) in the coil was measured to see the saturation point of the magnet. The magnet starts to saturate approximately at $2000 \mathrm{Amp}$ as can be seen in the solid curve on the top plot of figure 3.11. The dotted line is the 


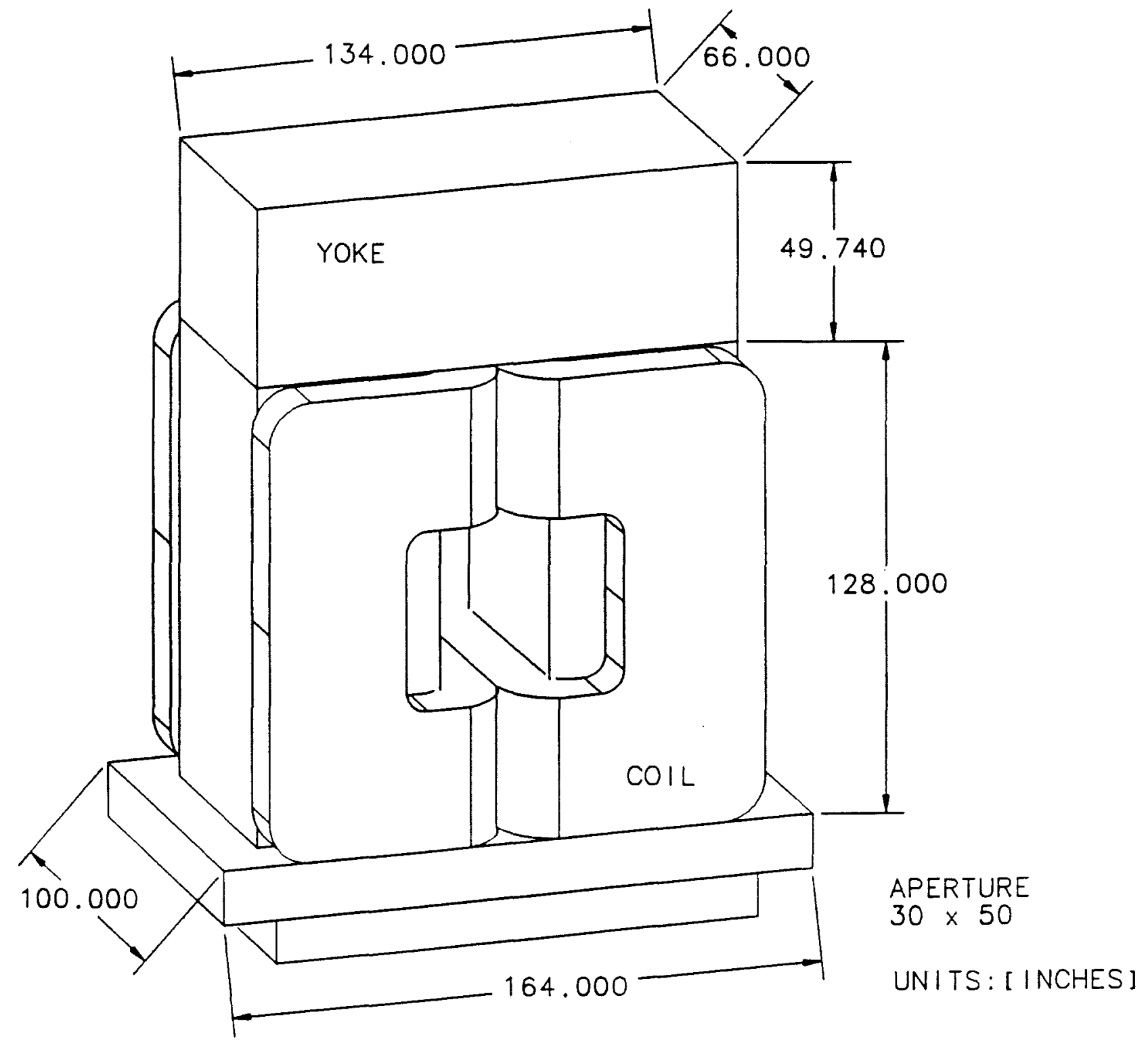

Figure 3.10: M2 Geometry 
linear fit at low current. $\vec{B}$ as a function of the distance (Z) from the center of the magnet was also measured (see bottom plot of figure 3.11). To stop the magnetic field from going outside of the magnet region (100 Inches thick) we installed 2 shields of steel (mirrors) upstream and downstream of the magnet. However, the magnetic field (from the current and from the steel) in the yoke was calculated, not measured, using a standard computer design program [38]. With a knowledge of the field anywhere (M2 region), the trajectory of the stub is traced solving the Lorentz equation [36] through this particular muon filter (yoke of the magnet).

The path of a charged particle of velocity $\vec{v}$ in a magnet of field $\vec{B}$ is governed by the well known Lorentz equation;

$$
\vec{F}=\frac{e}{c} \vec{v} \times \vec{B}
$$

where $\vec{F}$ is in CGS units system. Separating equation 3.8 by components we find the equations of the trajectory,

$$
\begin{aligned}
& x^{\prime \prime}=\frac{e}{P_{c}}\left[x^{\prime} y^{\prime} B_{x}-\left(1+x^{\prime 2}\right) B_{y}+y^{\prime} B_{z}\right] \sqrt{1+x^{\prime 2}+y^{\prime 2}} \\
& y^{\prime \prime}=\frac{e}{P_{c}}\left[\left(1+y^{\prime 2}\right) B_{x}-x^{\prime} y^{\prime} B_{y}-x^{\prime} B_{z}\right] \sqrt{1+x^{\prime 2}+y^{\prime 2}}
\end{aligned}
$$

where we transformed the components of $\vec{v}$ into spatial derivatives by using;

$$
\begin{aligned}
& \dot{x} \equiv v_{x}=\frac{d x}{d t}=\frac{d x}{d z} v_{z}=x^{\prime} v_{z} \\
& \ddot{x} \equiv \dot{v}_{x}=\frac{d x^{\prime}}{d t} v_{z}+x^{\prime} \frac{d v_{z}}{d t}=x^{\prime \prime} v_{z}^{2}+x^{\prime} \dot{v}_{z}
\end{aligned}
$$

and similarly for y components.

The second order differential equation 3.9 and 3.10 are simultaneously solved using the Runge-Kutta Method [39]. Taking the parameters of the incident stub, which are the position $\left(\mathrm{x}_{i}, \mathrm{y}_{i}\right)$, the slope $\left(\mathrm{x}_{i}{ }^{\prime}, \mathrm{y}_{i}{ }^{\prime}\right)$ and the momentum (P) at the mirror position $(z)$, these equations are numerically solved to find the parameter of the emergent stub ( $\left.\mathrm{x}_{e}, \mathrm{y}_{e}, \mathrm{x}_{e}^{\prime}, \mathrm{y}_{e}^{\prime}\right)$ from the magnet. The emergent stub is projected into the outer muon detector to predict the channel hit.

The trajectory of a particle through a magnetic field region of length $\mathrm{L}$ is a smooth circular arc. For small angles, the total deflection $\left(\theta_{B}\right)$ due to $\vec{B}$ (from equation 3.8 ) is;

$$
\theta_{B}=\frac{0.3}{P}|\vec{B}| L
$$



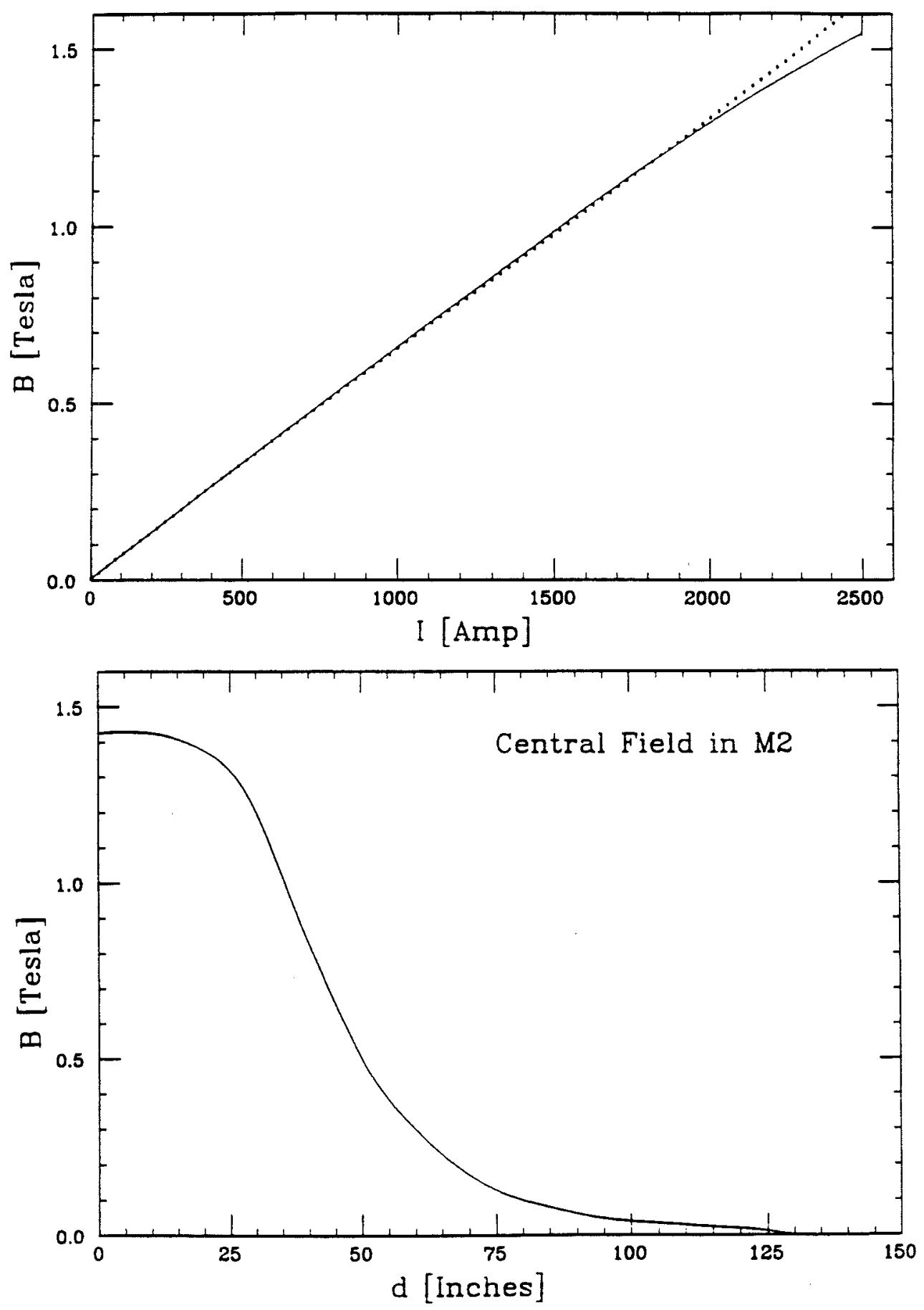

Figure 3.11: M2 Field 
where the units of $\vec{B}$ are Tesla, L are $m$ and $\mathrm{P}$ are $G e V / c$.

For muon identification purposes, the stub had to cross through the yoke to reach the outer detector. As we studied, its trajectory is affected due to the magnetic field in the steel and due to the multiple scattering in it. A fast calculation of the deflection of the stub due to the multiple scattering as a function of the momentum shows;

$$
\theta_{m s(y o k e)}=\frac{9.66}{P L} \text { Radians }
$$

where the length of the yoke $(\mathrm{L})$ is in Inches.

The mean angle of the deflection due to multiple scattering $\theta_{m s}$ (equation 3.1) in a given thickness of material, is inversely proportional to the kinetic energy of the particle. The deflection due to a magnetic field $\theta_{B}$ (equation 3.13) is only inversely proportional to the momentum. Therefore, at sufficiently low velocities, the multiple scattering effect will always be greater than the curvature in the magnetic field. In fact, neglecting the logarithmic term in equation 3.1, we have;

$$
\frac{\theta_{m s}}{\theta_{B}} \cong \frac{1}{20} \frac{1}{\sqrt{L_{r} L}} \frac{1}{\beta|\vec{B}|} .
$$

Even if the multiple scattering predominates, the magnetic field still gives some contribution and help to make the total curvature more uniform.

The stub that reach the outer detector not only crossed through the yoke, but also went through the Outer Electromagnetic Calorimeter (OE) placed upstream of M2. It is a detector composed of 5 blocks of lead-scintillator sandwich. Table 3.5 shows the total material upstream of the outer detector expressed in radiation length. The energy loss of the particle in these material was also calculated as we explained in the last section.

A analytical calculation of the total energy loss $\left(\mathrm{P}_{\text {Loss }}\right)$ and the multiple scattering $(\alpha)$ that the stub suffered before to hit the outer muon system is shown in table 3.6.

A muon beam run was not useful to measure the multiple scattering contribution to the error projection of the stub into the OMS. Muons coming from muon beam did not hit the microstrip detector (SSD). Then it is not possible to determine the momentum of the stub, therefore we can not solve the trajectory equation $(3.9,3.10)$. We used the results from our analytical calculation (table 3.6) to study the efficiency of the outer proportional tubes.

Knowing the errors of the projection of the tracks we are in condition to study the geometry of the proportional tube planes, study we will perform in the next section. 
Table 3.5: Upstream Detector of Outer System

\begin{tabular}{|l|c|c|c|}
\hline $\begin{array}{l}\text { Detector } \\
\text { Name }\end{array}$ & $\begin{array}{c}\text { Length } \\
C m\end{array}$ & $\begin{array}{c}\text { Radiation Length } \\
L_{r}\end{array}$ & $\begin{array}{c}\text { Energy Loss } \\
G e V\end{array}$ \\
\hline & & & \\
OE0 & 4.158 & 1.289 & 0.017 \\
OE9 & 7.524 & 1.163 & 0.023 \\
OE1 & 17.620 & 5.342 & 0.075 \\
OE2 & 17.620 & 5.342 & 0.075 \\
OE3 & 17.620 & 5.342 & 0.075 \\
YOKE & 167.640 & 95.250 & 1.953 \\
\hline
\end{tabular}

Table 3.6: Analytical "Outer" Calculation

\begin{tabular}{|c|c|r|}
\hline Path & & Outer \\
\hline Oe0-Oe9-Oe1 & & \\
\cline { 2 - 3 } $\begin{array}{c}\text { Oe2-Oe3- } \\
\text { Yoke }\end{array}$ & $\mathrm{L}_{r}$ & 113.73 \\
\cline { 2 - 3 } & $\mathrm{P}_{\text {Lose }}$ & 2.22 \\
\cline { 2 - 3 } & $\alpha$ & 14.60 \\
\hline
\end{tabular}

\subsection{Proportional Tubes Geometry}

Once we have understood the errors in projecting tracks to the muon system, we are in a position to find out the central coordinates of each proportional tube plane, the spacing between the tubes and the rotation of the plane with respect to the spectrometer reference frame.

As we did in section 3.4, we use the PWC's to project the track in each plane to measure the agreement between our projection and the data. Then we calculate $\chi_{j}^{2}$ over all the tracks $(i=1 \ldots n)$ for each plane $j$, that is

$$
\chi_{j}^{2}=\sum_{i=1}^{n}\left[\frac{X c_{i}^{j}-X p_{i}^{j}}{\sigma_{i}^{j}\left(P_{i}\right)}\right]^{2}
$$

where $\sigma_{i}^{j}$ is the multiple scattering error as a function of the track momentum $\left(\mathrm{P}_{i}\right)$ calculated on the two last sections. $X p_{i}^{j}$ is the straight line projection of the track $i$ in the plane $j . X c_{i}^{j}$ is the central coordinate of the closest tube on to the predicted one for plane $j$ and track $i$. Rewriting $X c$ as a function of the central tube number $\left(\omega_{c}\right)$, the predicted tube number $(\omega)$ and the spacing of the tubes in the plane $(\mathrm{d} j)$,

$$
X c_{i}^{j}=\left(\omega_{c}^{j}-\omega_{i}^{j}\right) d j
$$


and defining the corrections to the nominal values of the center of the plane $\left(\delta \omega_{c}\right)$ and the space between two tubes $(\delta d j)$, it is

$$
\begin{aligned}
\omega_{c}^{j} & ={\overline{\omega_{c}}}^{j}+\delta \omega_{c}^{j} \\
d j & =\overline{d j}+\delta d j
\end{aligned}
$$

where $\overline{\omega_{c}}$ is the nominal tube number placed in the center of the plane and $\overline{d j}$ is the nominal spacing of the tubes, which is 1.25 Inches in each plane.

The orientation of the plane $(\delta \theta)$ with respect to the reference frame is described by the well known rotation matrix,

$$
\left(\begin{array}{c}
X r \\
Y r
\end{array}\right)=\left(\begin{array}{rr}
\cos \delta \theta & \sin \delta \theta \\
-\sin \delta \theta & \cos \delta \theta
\end{array}\right)\left(\begin{array}{c}
X p \\
Y p
\end{array}\right)
$$

where $X r$ and $Y r$ are the rotated projected track in each plane. Assuming that the rotation $\delta \theta$ is small, we have

$$
\begin{aligned}
& X r=X p+Y p \delta \theta \\
& Y r=-X p \delta \theta+Y p .
\end{aligned}
$$

As we mentioned in an earlier section (section 3.2) the tubes in the planes labeled with $\mathrm{X}$ are placed vertically while the tubes are placed horizontally for planes labeled with $Y$. Therefore, we used equation 3.21 to project the track in plane labeled with $\mathrm{X}$ and equation 3.22 for planes labeled with $\mathrm{Y}$. For simplicity we define,

$$
\chi_{p}^{j}=X p^{j}+\tilde{X}^{j} \delta \theta^{j}
$$

where $X p^{j}$ and $\tilde{X}^{j}$ are the coordinates of the projection $\mathrm{x}$ and $\mathrm{y}$ respectively in planes $j=\mathrm{OMX}$, IM1X, IM2X. In planes $j=\mathrm{OMY}, \mathrm{IM} 1 \mathrm{Y}$, IM2Y the projected values $X p^{j}$ and $\tilde{X p}^{j}$ represent $\mathrm{y}$ and $\mathrm{x}$ respectively.

Including the translation and rotation corrections in $\chi_{j}^{2}$, we got it as a function of the spacing, central tube number and rotation parameters,

$$
\chi_{j}^{2}=\sum_{i=1}^{n}\left[\frac{\left({\overline{\omega_{c}}}^{j}-\omega_{i}^{j}\right) \overline{d j}-X p_{i}^{j}+\left({\overline{\omega_{c}}}^{j}-\omega_{i}^{j}\right) \delta d j+\overline{d j} \delta \omega_{c}^{j}-\tilde{X} p_{i}^{j} \delta \theta^{j}}{\sigma_{i}^{j}\left(P_{i}\right)}\right]^{2}
$$

where we have replaced equations $3.17,3.18,3.19,3.23$ in equation 3.16 and we neglected the small term $\delta \omega_{c}^{j} \delta d j$. Now, we want to minimize with respect to the parameters.

Using the Method of Least Squares the optimum values for the parameters are those for which the function $\chi^{2}$ is a minimum, i.e., for which 
the derivatives with respect to the parameters are 0 . This method had been well explained and described in [35] considering $\chi^{2}$ for an arbitrary function of order $m$,

$$
\chi^{2}=\sum_{i=1}^{n}\left[\frac{y_{i}-\sum_{l=0}^{m} a_{l} X_{l}\left(x_{i}\right)}{\sigma_{i}}\right]^{2}
$$

where $a_{l}$ are the parameters to determine. Minimizing with respect to each coefficients, the system of $m+1$ equations is found

$$
\sum_{l=0}^{m} a_{l} \alpha_{l k}=\beta_{k}
$$

where the solution for the coefficients are,

$$
a_{l}=\sum_{k=0}^{m} \epsilon_{l k} \beta_{k}
$$

The matrix elements $\alpha, \beta$ and $\epsilon$ are;

$$
\begin{aligned}
\alpha_{l k} & =\sum_{i=1}^{n} \frac{X_{l}\left(x_{i}\right) X_{k}\left(x_{i}\right)}{\sigma_{i}^{2}} \\
\beta_{k} & =\sum_{i=1}^{n} \frac{y_{i} X_{k}\left(x_{i}\right)}{\sigma_{i}^{2}} \\
\epsilon_{l k} & =\frac{(-1)^{k+l} \alpha_{k l}}{|\alpha|}
\end{aligned}
$$

where $|\alpha|$ is the determinant. The error in the determination of the parameters are $\sigma_{a_{l}}^{2}=\epsilon_{l l}$.

To find out our corrections $\left(\delta d j, \delta \omega_{c}\right.$ and $\left.\delta \theta\right)$ we substitute;

$$
\begin{aligned}
& y_{i}=\left(\overline{\omega_{c}}-\omega_{i}^{j}\right) \overline{d j}-X p_{i}^{j} \\
& a_{0}=\delta d j \quad X_{0}=-\left(\bar{\omega}_{c}^{j}-\omega_{i}^{j}\right) \\
& a_{1}=\delta \omega_{c}^{j} \quad X_{1}=-\overline{d j} \\
& a_{2}=\delta \theta^{j} \quad X_{2}=\bar{X}_{p_{i}^{j}}
\end{aligned}
$$

in equation 3.25 and we calculate the values of these parameters using equation 3.27. This calculation was made for the inner muon proportional tube planes and the results are shown in table 3.7. The errors in each parameter are of the order of $10^{-4}$. We can observe that the correction in the spacing and the rotation of the plane are very small in each plane, therefore, we set these correction to the nominal values $d j=1.25$ Inches and $\theta=0$ Radians. The 
Table 3.7: Inner Muon Proportional Tubes Corrections

\begin{tabular}{|l|c|c|c|}
\hline Detector & $\delta \omega_{c}$ & $\delta d j$ & $\delta \theta$ \\
Name & Tubes & Inches & Radians \\
\hline IM1X & $-7.598 \times 10^{-2}$ & $-1.385 \times 10^{-2}$ & $3.557 \times 10^{-3}$ \\
IM1Y & $3.485 \times 10^{-2}$ & $2.123 \times 10^{-4}$ & $3.834 \times 10^{-3}$ \\
IM2X & $-1.547 \times 10^{-2}$ & $-6.442 \times 10^{-3}$ & $1.795 \times 10^{-3}$ \\
IM2Y & -0.200 & $-7.078 \times 10^{-3}$ & $-1.943 \times 10^{-2}$ \\
\hline
\end{tabular}

Table 3.8: Proportional Tube Planes Shift

\begin{tabular}{|l|c|c|}
\hline $\begin{array}{l}\text { Detector } \\
\text { Name }\end{array}$ & $\begin{array}{c}\text { Shift on X } \\
\text { Inches }\end{array}$ & $\begin{array}{c}\text { Shift on Y } \\
\text { Inches }\end{array}$ \\
\hline IM1X & -0.095 & 0.0 \\
IM1Y & 0.0 & 0.044 \\
IM2X & -0.019 & 0.0 \\
IM2Y & 0.0 & -0.251 \\
OMX & 0.0 & 0.0 \\
OMY & 0.0 & 0.0 \\
\hline
\end{tabular}

tube center correction multiplied by the nominal spacing gives the shift of the plane, shown in table 3.7. Due to the low multiplicity in the outer muon proportional tubes, we could not determine these correction. Then we assumed that these detectors are centered with respect to M2.

Now that we have a good understanding of the geometrical correction in the muon system and the error of the projection of the tracks due to the multiple scattering, we can determine the efficiency of these detectors.

\subsection{Efficiency Measurements}

The efficiency in the muon system was measured using the correct geometry calculated in the previous section. The reconstructed tracks were projected to each plane. If the latch bit was on when the channel was matched by the track we called channel "fired". Then the efficiency was defined,

$$
\text { Eff } f_{\text {Channel }}=\frac{\text { Number of tracks that fire the channel }}{\text { Number of tracks pointing to the channel }} .
$$

Based on the error of the track due to the multiple scattering $\left(\sigma_{t}(P)\right)$, we defined a cone of radius $3 \sigma_{t}(P)$ around the straight line projection. Because 
Table 3.9: Average Efficiency

\begin{tabular}{|l|c|}
\hline $\begin{array}{l}\text { Detector } \\
\text { Name }\end{array}$ & $\begin{array}{c}\text { Efficiency } \\
\%\end{array}$ \\
\hline IM1V & 99.760 \\
IM1H & 99.863 \\
IM2H & 99.816 \\
IM1X & 85.758 \\
IM1Y & 84.081 \\
IM2X & 81.401 \\
IM2Y & 82.549 \\
\hline
\end{tabular}

the probability for a measurement to fall within three standard deviation of the mean is about $99.7 \%$ [35] we used the factor 3 . We predicted channels inside of the cone projecting the track

$$
\begin{aligned}
& X=X p+3 \sigma_{t} \cos \Theta \\
& Y=Y p+3 \sigma_{t} \sin \Theta
\end{aligned}
$$

where the first terms ( $X p$ and $Y p$ ) are the projections considering there was no material in front of the muon detector and the second terms are the multiple scattering contribution. The polar angle $\Theta$ goes from 0 to $2 \pi$ to look at all the channels within the cone. If any of these channels were on, we counted it as a track that fired.

We measured the efficiency of each channel taking tracks to match in IMS and stubs to match in the OMS. The average efficiency per plane are shown in table 3.9 where we neglected the OMS planes because we did not have enough statistics to do the measurements.

The inefficiency values for each tube in the IMS are shown in figure 3.12 where we plotted the fraction of times that we predicted a channel off.

Now that we have an idea of the characteristics of the muon system, we can establish a criterion to identify muons, as we shall see in the next section.

\subsection{Muon Identification}

As we did in previous sections, we predict a channel fired by projecting the track in each plane and by counting the number of planes matched to define it as a muon. 

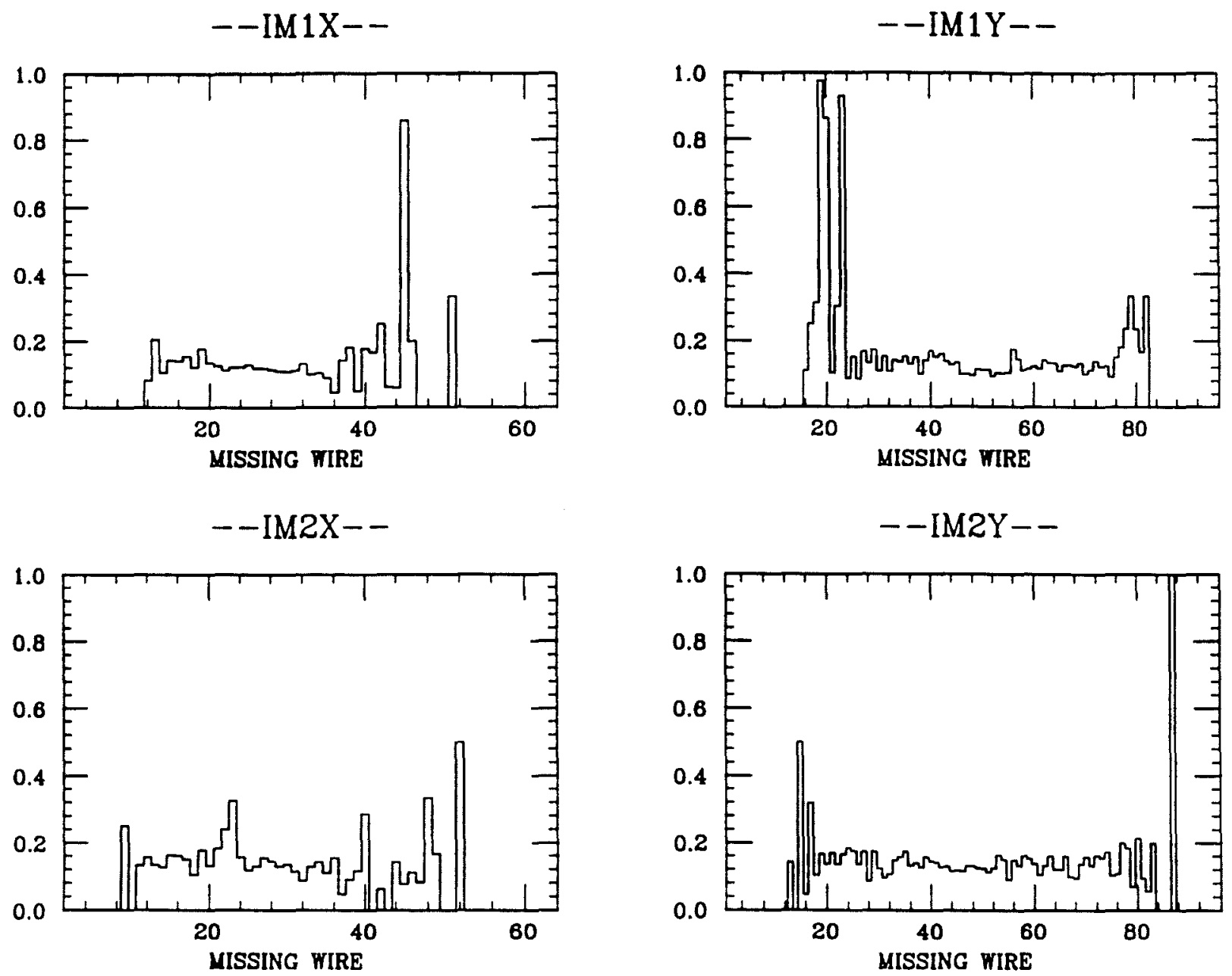

Figure 3.12: Inefficiency 
Table 3.10: Probability of Missing Planes

\begin{tabular}{|c|l|}
\hline $\begin{array}{c}\text { Missing } \\
\text { Planes }\end{array}$ & $\begin{array}{c}\text { Probability } \\
\%\end{array}$ \\
\hline 0 & 48.18 \\
1 & 38.58 \\
2 & 11.59 \\
3 & 1.56 \\
4 & $8.17 \times 10^{-2}$ \\
5 & $4.27 \times 10^{-4}$ \\
6 & $7.60 \times 10^{-7}$ \\
7 & $4.45 \times 10^{-10}$ \\
\hline
\end{tabular}

We can get a rough idea of the muon identification (muon id) criterion, by computing the probability to have exactly $n$ planes missing for a track travelling through $i$ planes of known efficiency. This probability is a sum of terms, one term for each of the ways that one can have $n$ planes missing. Each term is a product of factors, one factor for each of the planes. This factor is the plane efficiency, $\epsilon_{i}$, if the plane is not missing or $\left(1-\epsilon_{i}\right)$ if the plane is missing.

Taking the IMS average efficiencies shown in table 3.9, we calculated the probability of having a specific number of planes missing, results that are shown in table 3.10. From these numbers and in order to establish a muon id definition, we calculated the probability of having a minimum number of matched planes. In table 3.11 we show the calculation of the probability of detecting two, one or zero muon, assuming 2 track events. From this table we can see that the muon id definition 5 out of 7 planes can be a reasonable requirement for a track to be a muon identified in the inner detector.

Due to the unknown efficiency in the OMS, we can not calculate its probability. A simple muon id criterion for the stub can be 1 out of 4 planes accordingly with the outer trigger studied in a previous chapter.

To decide which criterion we will use to select muons, we studied a $J / \Psi$ (goes to $\mu^{+} \mu^{-}$) sample of the dimuon trigger data taken. Selecting two opposite charge tracks events and assuming that both are muon, we calculated the invariant mass $\left(M_{\mu^{+} \mu^{-}}\right)$of the sample to be analyzed (figure 3.13). The events in this sample come from the Inner-Inner or Inner-Outer trigger explained in a preceding chapter. Taking 2 track events from this sample, we calculated the invariant mass of 2 muons identified with 3 different muon id definitions (figure 3.14), 6/7 (top row), 5/7 (central row) and 4/7 (bottom row). 
Table 3.11: Probability of Matching

\begin{tabular}{|c|c||c|c|c|}
\hline $\begin{array}{c}\text { Matched } \\
\text { Planes }\end{array}$ & $\begin{array}{c}\text { Probability } \\
\%\end{array}$ & $\begin{array}{c}\text { Prob. 2 } \mu \\
\%\end{array}$ & $\begin{array}{c}\text { Prob. 1 } \mu \\
\%\end{array}$ & $\begin{array}{c}\text { Prob. 0 } \mu \\
\%\end{array}$ \\
\hline 7 & 48.18 & 23.21 & 49.93 & 26.85 \\
6 & 86.77 & 75.29 & 22.96 & 1.75 \\
5 & 98.36 & 96.75 & 3.22 & $2.68 \times 10^{-2}$ \\
4 & 99.92 & 99.83 & $1.64 \times 10^{-1}$ & $6.74 \times 10^{-5}$ \\
3 & 99.99 & 99.99 & $8.58 \times 10^{-4}$ & $1.84 \times 10^{-9}$ \\
\hline
\end{tabular}

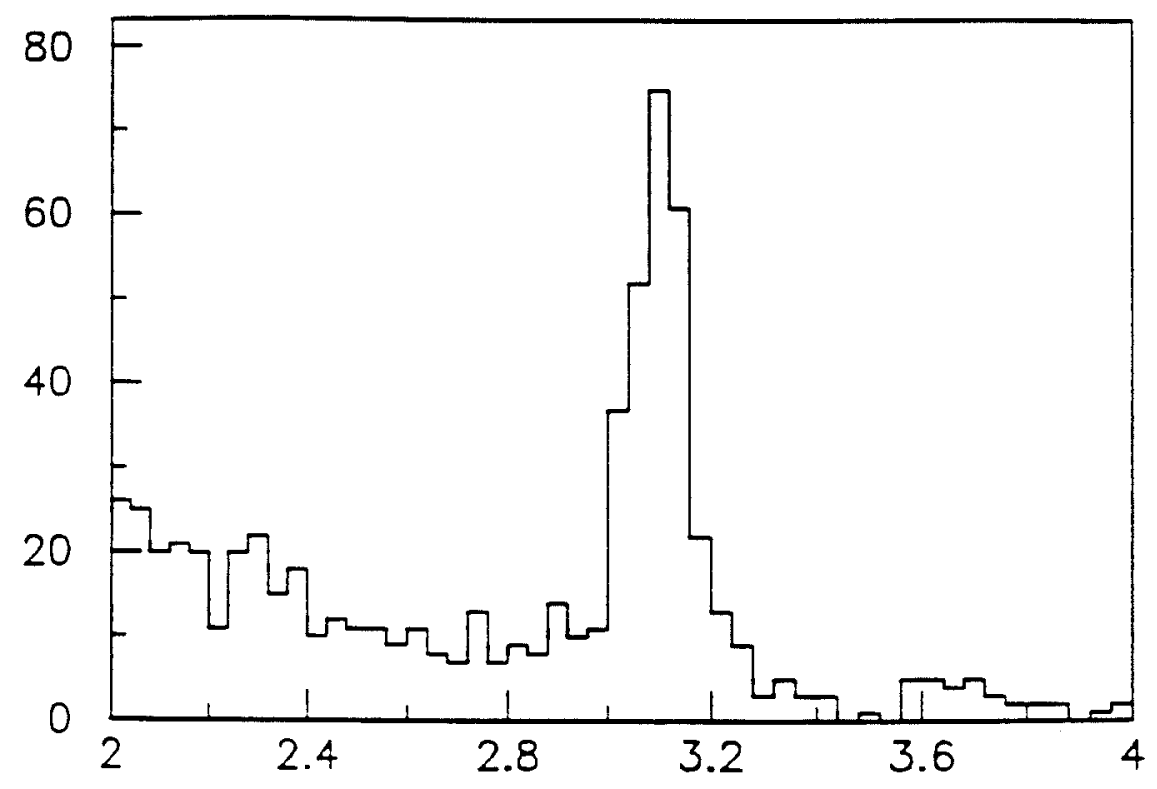

Figure 3.13: Muon Trigger $J / \Psi$ Sample (Bin of $40 \mathrm{MeV}$ ) 

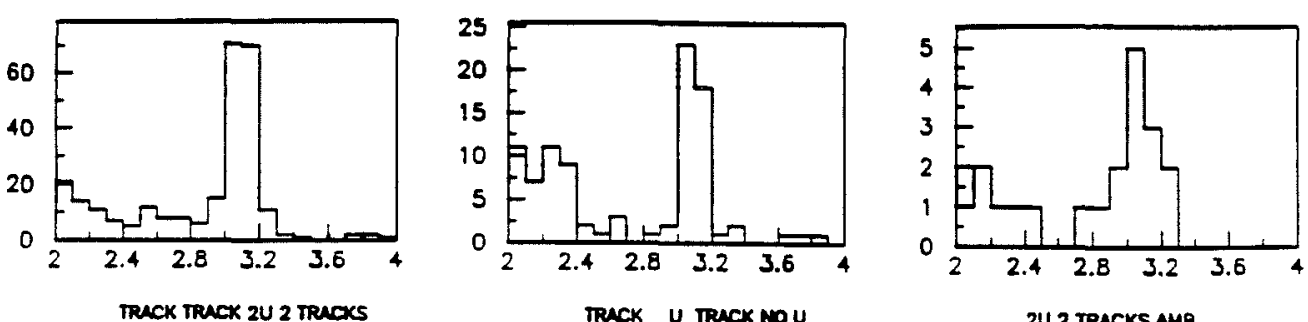

50
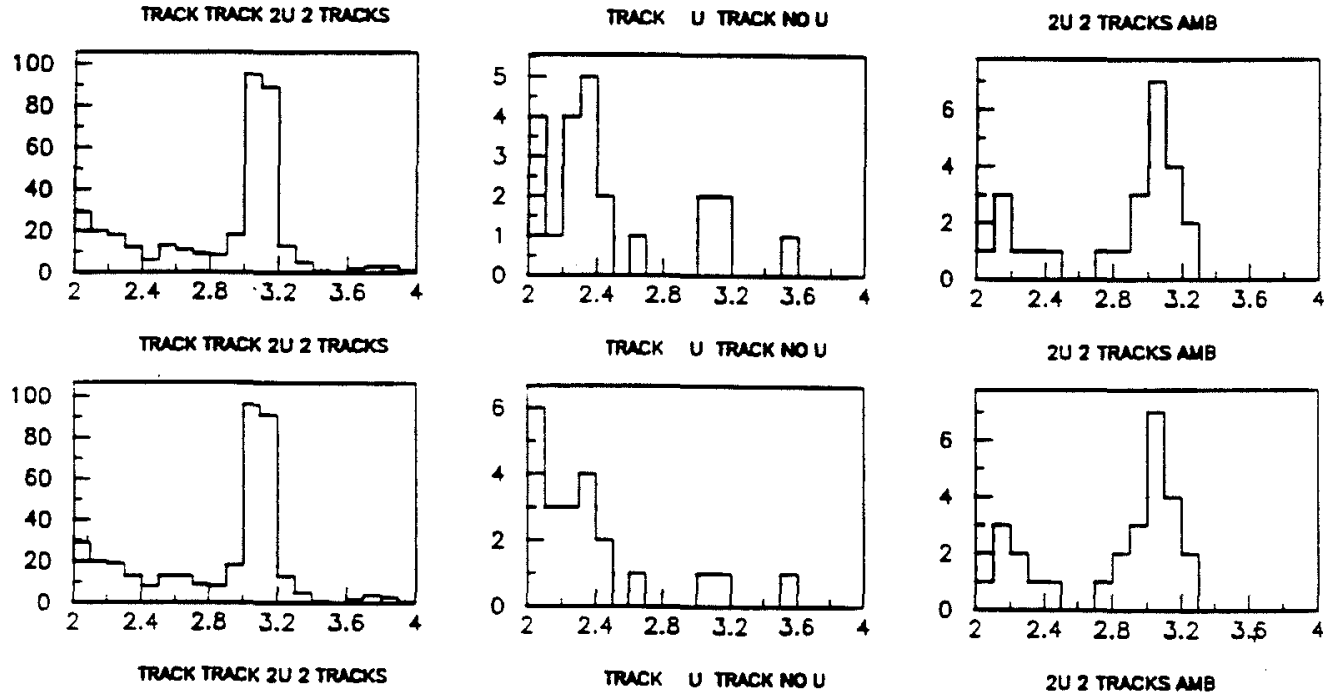

Figure 3.14: Muon Identification Sample (Bin of $100 \mathrm{MeV}$ )

Next we computed the ratio background/signal to decide for the best muon id criterion. Three different measurements are shown in figure 3.14 where we included two more sets of invariant mass calculation of the event. The central column shows the invariant mass when only one tracks is identified as a muon while the last column shows when the identification of the muon is ambiguous, it meant that the track share more than one channel fired with other tracks pointing to the muon system. As we can see in figure 3.14, the $M_{\mu^{+} \mu^{-}}$of the requirement 5 out of 7 show a good ratio background/signal, reason for which we took it as a muon id criterion. In other words, this definition required at least one fired channel in the back planes of the IMS.

To see how well this definition work, we took a muon beam run and we calculated the muon id efficiency dividing the number of identified muons with this criterion $(5 / 7)$ by the total number of tracks pointing to the inner detector; its value is $97.85 \%$. This value agrees with the theoretical calculation of the probability show in table 3.11. Also we measured (figure 3.15) the muon id efficiency as a function of the momentum of the incident track.

Finally, a muon data selection of all dimuon trigger data taken (246 


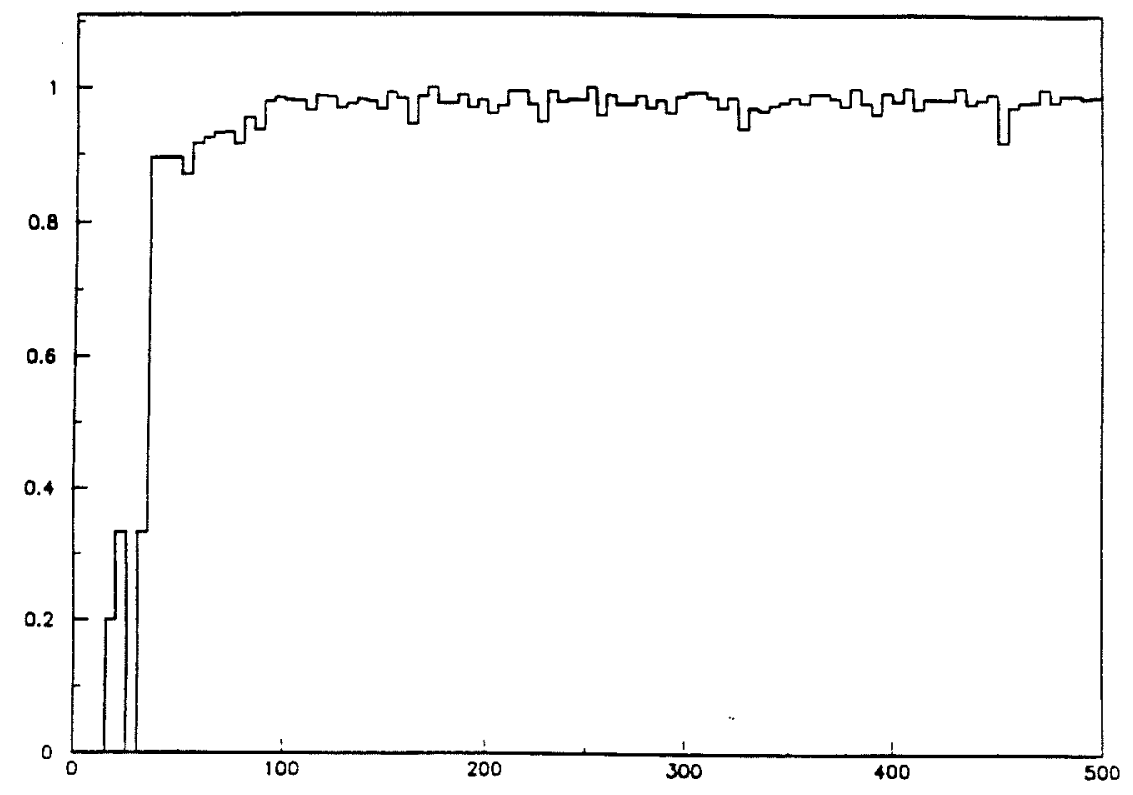

Figure 3.15: Muon Id Efficiency

tapes) was done using the muon id definition studied in this section. Furthermore this data selection was performed to select $J / \Psi \rightarrow \mu^{+}+\mu^{-}$candidates in events with at least two reconstructed tracks in the spectrometer. In order to get a clean selection we required additional cuts. A vertex in the target region and opening angle greater than 0.001 radians was required in events with 2 tracks. At least one vertex was required in multitracks events.

The total sample gathered from this skim is shown in the invariant mass histograms (bin of $0.15 \mathrm{GeV}$ ) of the dimuon events for 2 tracks and for multitracks events (figure 3.16 ).

Now that we have obtained the $J / \Psi$ signal, we will study it in much more detail in the next chapter. 

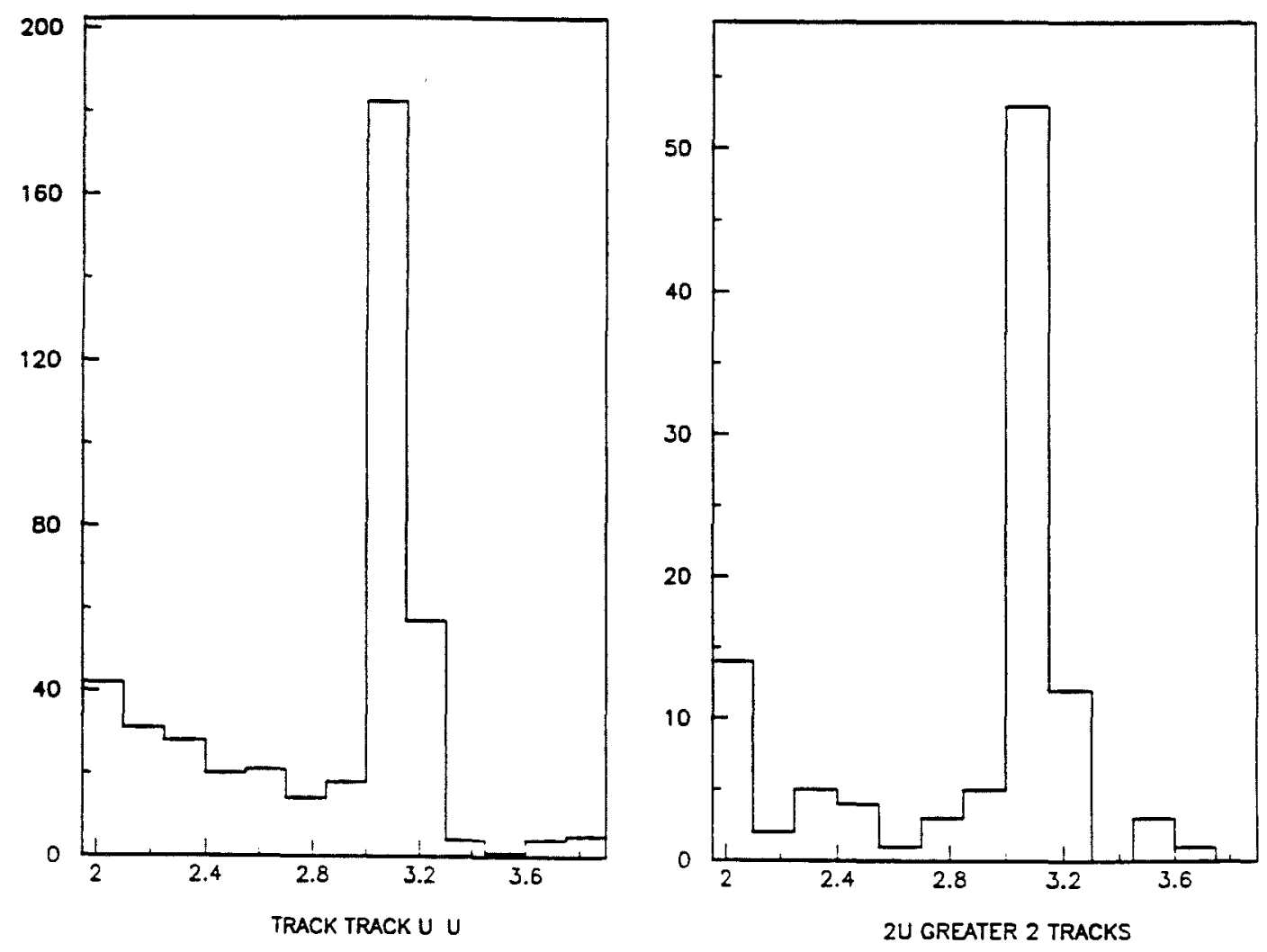

Figure 3.16: Muon Data Selection Results 


\section{Chapter 4}

\section{Data Analysis and Conclusions}

\subsection{Introduction}

With the advent of high intensity, high energy photon beams, theorists have shown renewed interest in the use of QCD models to describe the photoproduction states containing open and bound heavy quarks $(Q)$. The large mass of the charm $(c)$ and bottom $(b)$ quark result in small enough values of $\alpha_{s}\left(m_{Q}^{2}\right)$ to justify the use of perturbative methods. The lowest order QCD process is the photon gluon fusion diagram [16], described in a previous chapter of this report. The total cross section $(\gamma+n u c l e o n \rightarrow Q \bar{Q})$ predicted by this model is sensitive to the gluon distribution function $(F(x))$ and to the quark mass $\left(m_{Q}\right)$ assumed. Once $F(x)$ has been chosen, the computation of the cross section as a function of the photon energy is reduced basically to the specification of the mass threshold production. In the case of the charm bound states, as $J / \Psi$, this threshold is $\left(2 m_{c}\right)^{2}$ and the upper limit $\left(2 m_{D}\right)^{2}$ with $m_{D}$ as the $D^{0}$ meson mass [18].

Recent efforts of experimenters have focused on verifying the expectations of this model for the $J / \Psi$ cross section [40]. The elastic and inelastic cross section of the $J / \Psi$ photoproduction has been measured by several groups using different targets at energies between $11 \mathrm{GeV}$ to $275 \mathrm{GeV}$ $[40,41,42,43,44,45,46,47,48]$.

In this chapter, we present the elastic $J / \Psi$ cross section measurements on a beryllium target at energies from $100 \mathrm{GeV}$ to $400 \mathrm{GeV}$ (see section 4.5). Several steps were taken to extract the cross section from the raw data. The first step consists of selecting the $J / \Psi$ sample. This sample is obtained by requiring two opposite sign muons in the final state. Several cuts were applied to clean the sample and to subtract the background, for example, kinematics and vertex constraints, linked tracks, good spills, etc., (see section 4.2). The 
next step in the calculation is to compute the acceptance of the spectrometer. It was calculated using a Monte Carlo program (Geant3 [33]) where we have simulated the inefficiencies of the detectors, the incident beam profile, the physical aperture of the detector (geometric acceptance), the kinematics of the process and the physical processes involved in the detection of the sample, as for instance, multiple scattering, energy loss, etc., (see section 4.4). The final step is the absolute normalization. We used the integrated flux of the electrons from which the photon flux is deduced (see section 4.3). Then, using these results, we measured the cross section $\left(\sigma_{\gamma N \rightarrow J / \Psi N}\right)$ as a function of the interacting photon energy.

Finally, these results are compared in shape and magnitude with other experimental results and with the predictions of the photon gluon fusion mechanism.

\subsection{Data Reduction}

The data sample was derived from all $(\sim 1100)$ post-fire raw data tapes by requiring that the events satisfy the minimum bias, pair or muon trigger bits, reducing the sample to 217 tapes. These tapes were analyzed to reconstruct the tracks in the spectrometer, resulting in 246 new output tapes [49]. Next, a skim was developed to look for $J / \Psi$ in the $\mu^{-} \mu^{+}$decay mode. Several conditions were needed to select the sample. We produced 33 tapes with the events that passed the following cuts:

1.- Events coming from the Inner-Inner or Inner-Outer muon trigger,

2.- Events with at least two tracks reconstructed by the PWC system,

3.- Events with at least two identified muons,

4.- Events with at least one vertex,

5.- Events with two muons opposite charged and

6.- Dimuon events with two tracks only, and having an opening angle $\geq 0.001$ radians.

A clear $J / \Psi$ signal was obtained. We calculated the invariant mass of the dimuon sample for "elastics" and "inelastic" events (figure 3.16). Events were classified as elastic if no charged or neutral track accompanied the dimuon event (two PWC tracks), otherwise, the event was classified as inelastic (multitracks).

As our emphasis was on measuring the elastic $J / \Psi$ cross section $(\sigma)$ in beryllium as a function of the interacting photon energy $\left(E_{\gamma}\right)$, we concentrate only on the elastic dimuon sample. The inelastic sample will not be discussed in this report. 


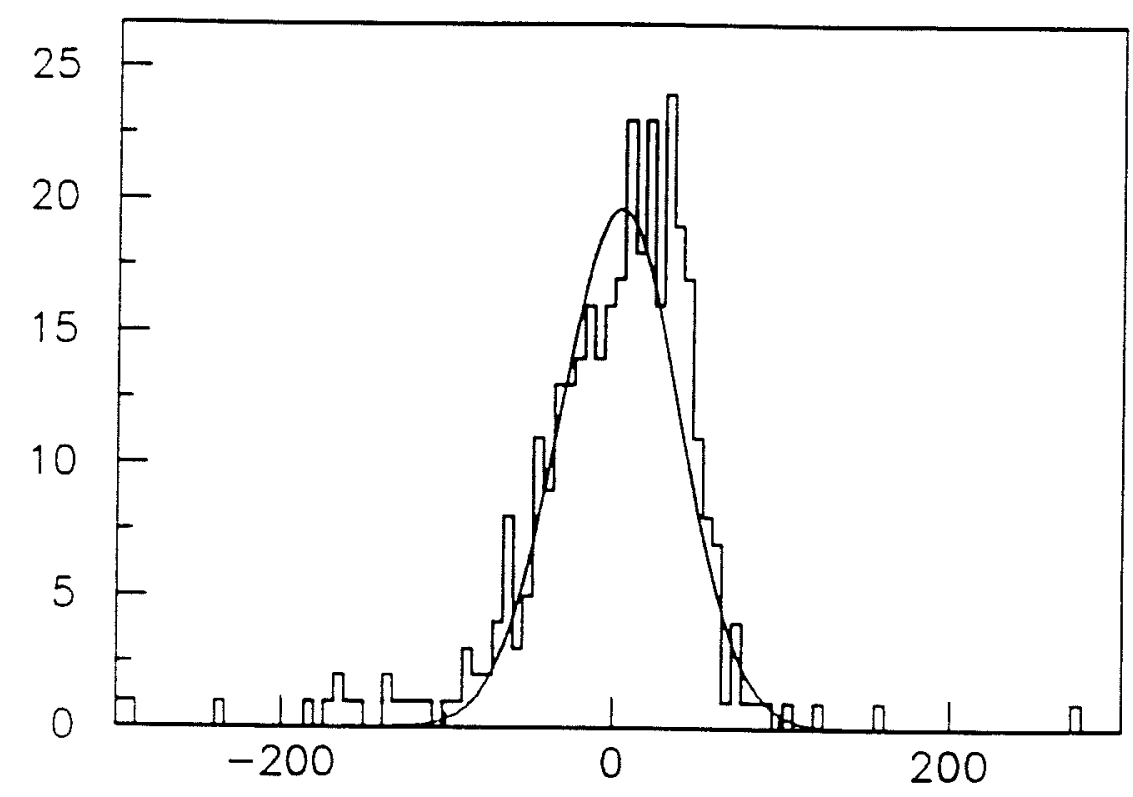

Figure 4.1: $\Delta E=E_{\gamma}-E_{J / \Psi}$

The measurements of $E_{\gamma}$ involve a knowledge of the incident electron beam energy (equation 2.6), which in our case is $350 \mathrm{GeV}$ with an error of $15 \%$. In fact, equation 2.6 is affected by a big error due to the uncertainties in the electron beam energy and in the energy resolution of the tagging system. A better approximation of $E_{\gamma}$, instead of equation 2.6 , can be obtained by the $J / \Psi$ energy $\left(E_{J / \Psi}\right)$ in elastic events [50], where $E_{\gamma}=E_{J / \Psi}$ is a good level of approximation as can be seen in figure 4.1. This figure shows the difference between the measured $E_{\gamma}$ by our tagging system and the $E_{J / \Psi}$ as well as the gaussian fit represented by the continuous line. This fit demonstrates the error $\left(\sigma_{f i t}=36.1 \pm 1.7 \mathrm{GeV}\right)$ in the determination of $E_{\gamma}$ is within the boundaries of the quoted error above. Henceforth, we will use $E_{J / \Psi}$ as $E_{\gamma}$, the gamma energy.

The elastic sample was analyzed to extract a final sample from which the cross section is measured. This final sample was obtained as a result of applying several cuts designed to clean the sample and to subtract the background yielding the $J / \Psi$ sample. Cuts in the trigger, target and tracking were employed. Events that came from the Inner-Outer trigger were not considered due to the unknown efficiency of the outer muon system (previous chapter), consequently, we do not use the events which contain stubs ( 3 chambers tracks). Only the good running period (defined in section 4.3) in which there was 5 pieces of beryllium target was used. In addition, linking and vertex constraints were required to eliminate poorly measured tracks. This means that 
Table 4.1: $J / \Psi$ Sample Fit

\begin{tabular}{|c|c|c|c|}
\hline & $\begin{array}{c}\text { Mass } \\
G e V\end{array}$ & $\begin{array}{c}\sigma_{M} \\
G e V\end{array}$ & $\begin{array}{c}\text { Number of Events } \\
\text { in the Peak }\end{array}$ \\
\hline$J / \Psi$ & $3.094 \pm 0.006$ & 0.06 & $133.3 \pm 12.5$ \\
\hline
\end{tabular}

the two PWC tracks (5 or 4 chambers tracks) identified as muons are required to be linked with tracks found by the SSD detector, which at the same time, made a vertex in the target region. No other tracks were permitted to come from the same vertex. Dimuon events with an invariant mass $\left(M_{\mu \mu}\right)$ within 1 $\mathrm{GeV}$ of the nominal mass of the $J / \Psi\left(M_{J / \Psi}=3.096 \mathrm{GeV}\right)$ were used to define the $J / \Psi$ candidate sample. Summarizing, we kept for the subsequent analysis those events that satisfied the following cuts:

1.- Events that come from the Inner-Inner muon trigger,

2.- Events that come from only 5 pieces of beryllium target period,

3.- Events that come from good spill period,

4.- Both muons linked with SSD tracks,

5.- Both muons come from a common vertex,

6.- Vertex coordinates inside of the target region and

7.- Dimuon invariant mass with $\left|M_{\mu \mu}-M_{J / \Psi}\right| \leq 1 \mathrm{GeV}$.

A total of 234 dimuon events were obtained which satisfied the above mentioned conditions. The invariant mass of these events are shown in figure 4.2 where we have fitted the resonance by a gaussian function over a smooth exponential background getting 133 events in the peak. The result of the fit is shown in table 4.1 .

Next, we cut the sample in $\pm 3 \sigma$ around the peak found in the previous fit, finding 151 events which contain approximately $12 \%$ of background. This background is composed essentially of electromagnetic production (BetheHeitler). These events can be characterized by low momentum transfer, $t$, which in this case is the transverse squared momentum of the $J / \Psi$ shown in figure 4.3. A big fraction of this background is subtracted by imposing a cut of $t \leq 0.02 \mathrm{GeV}^{2}$ (see section 4.5). The energy distribution of the 124 events that passed the $t$ cut is presented in figure 4.4 and represents our final $J / \Psi$ sample. Finally this sample is divided into photon energy bins of $50 \mathrm{GeV}$ each (table 4.2). 


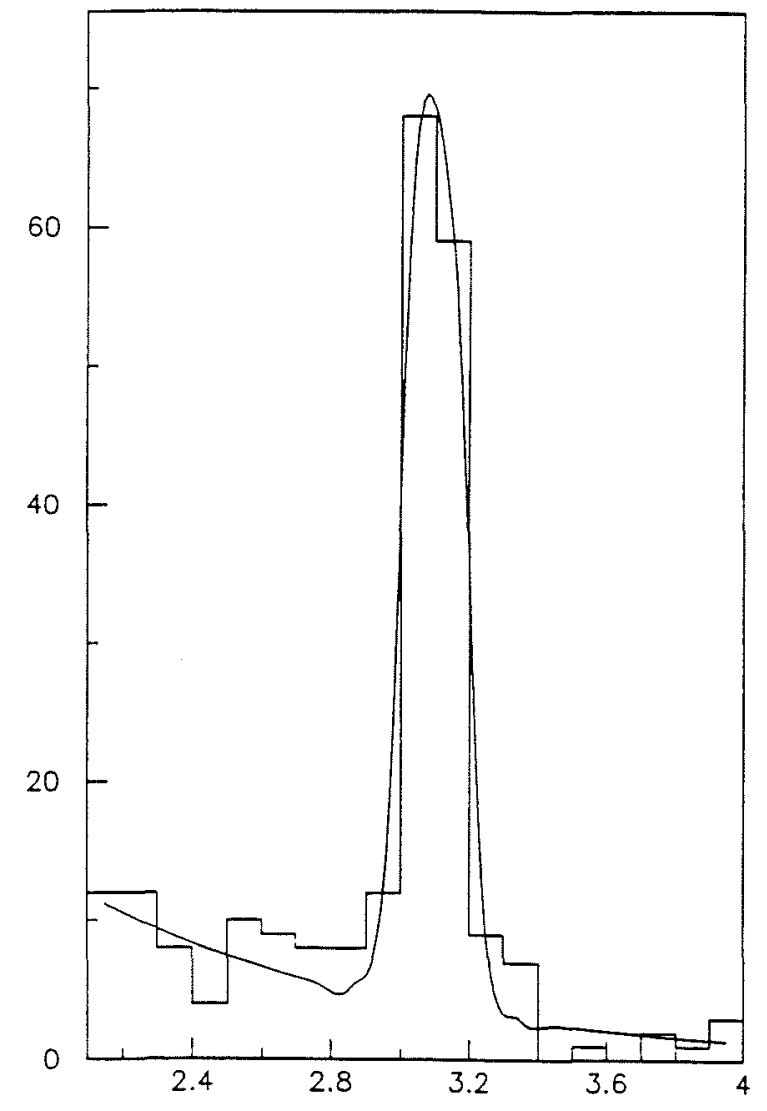

57

Figure 4.2: $J / \Psi$ Candidates Sample

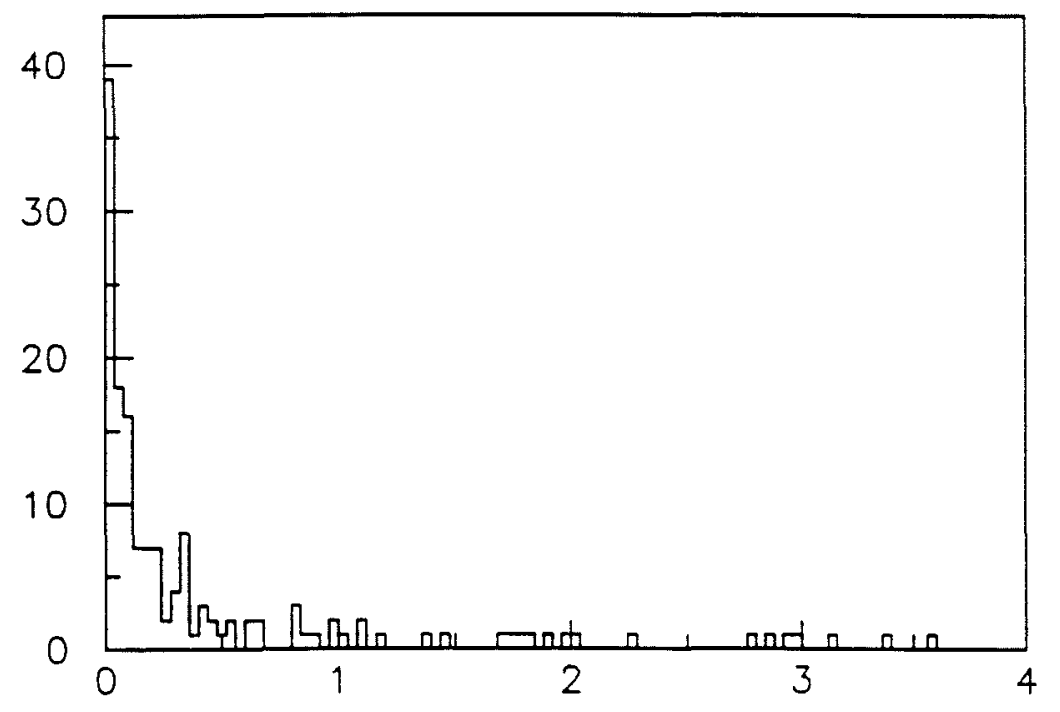

Figure 4.3: $J / \Psi \quad t\left(=P_{T}^{2}\right)$ Distribution 


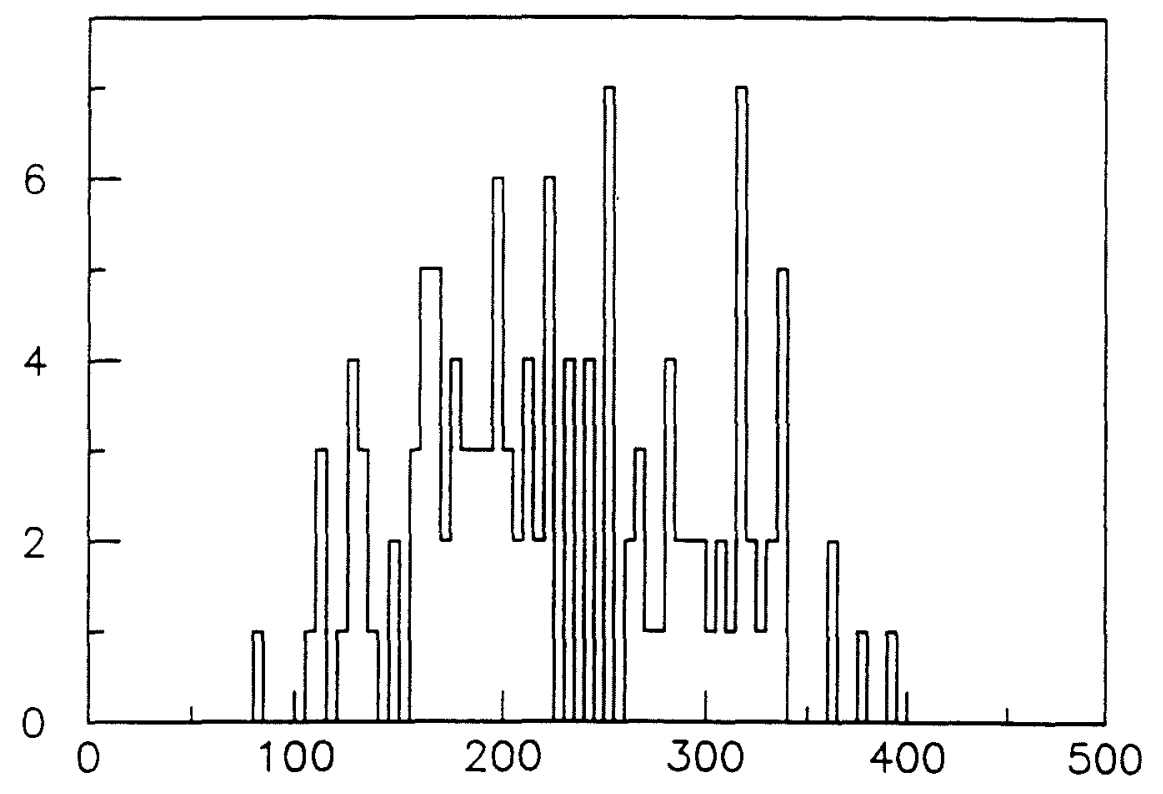

Figure 4.4: $J / \Psi$ Energy Distribution

Table 4.2: Bining Sample

\begin{tabular}{|c|c|}
\hline $\begin{array}{c}\Delta E_{\gamma} \\
G e V\end{array}$ & Events \\
\hline $100-150$ & 15 \\
$150-200$ & 34 \\
$200-250$ & 25 \\
$250-300$ & 24 \\
$300-350$ & 21 \\
$350-400$ & 4 \\
\hline
\end{tabular}




\subsection{Normalization}

In order to normalize the data, the number of nuclei per unit of area at the target and the beam flux must be known. The number of nuclei in the target are given by

$$
N_{A}=\frac{N_{0} \cdot \rho \cdot d_{e f f}}{A}
$$

where $N_{0}$ is the avogadro number; $\rho, A$ and $d_{\text {eff }}$ are the density, atomic weight and effective length of the target respectively. The effective length is defined by

$$
d_{\text {eff }}=\varepsilon_{\text {tar }} \cdot d
$$

where $d$ is the physical length of the target and $\varepsilon_{\text {tar }}$ is the targeting efficiency defined as the ratio of the total number of interaction in the target and the total number of incident particles. The number of interactions in the target was calculated using a Monte Carlo program where we have simulated a primary vertex coordinate and the incident beam profile. The beam profile has been reproduced by a 2-dimensional gaussian function in the transverse direction and a flat function along the beam.

We extracted our sample from the $B e$ target period. This target consists of 5 blocks of $B e$ (figure 2.8) of total length of $d=4.064 \mathrm{~cm}$, without taking into account the air gap between each block. Due to the shape of the target, in the calculation of $\varepsilon_{\text {tar }}$ we threw away the events with the interaction point in the gap of the target. The longitudinal profile of the target can be seen by plotting the longitudinal coordinates of the primary vertices reconstructed by the SSD. As we see in figure 4.5 there is a good agreement between the data and the Monte Carlo generated vertices. The peak at $2.5 \mathrm{~cm}$ in figure 4.5 corresponds to the counter TR1. With the above procedure we found $\varepsilon_{\text {tar }}=54.35 \%$.

Using $\rho_{B_{e}}=1.848 \mathrm{gr} / \mathrm{cm}^{3}, A_{B_{e}}=9.01 \mathrm{gr} / \mathrm{mol}, N_{0}=6.022 \times 10^{23}$ $\mathrm{mol}^{-1}$ and $d_{\text {eff }}=2.21 \mathrm{~cm}$, we calculated the total number of nuclei per unit of area to be $N_{A}=2.72 \times 10^{23} \mathrm{~cm}^{-2}$.

The incident beam "flux" at the target is given by

$$
\Phi_{t}=N_{A} \cdot N_{\gamma}
$$

where $N_{\gamma}$ is the total number of incident photons deduced from the integrated flux of incident electrons simulated and from the BGM scaler data. The BGM scaler incremented when the total energy seen by BGM in an event is greater than $133 \mathrm{GeV}$. Then, the number of photons can be written as

$$
N_{\gamma}=f_{c}\left(E_{\gamma}\right) \cdot B G M
$$



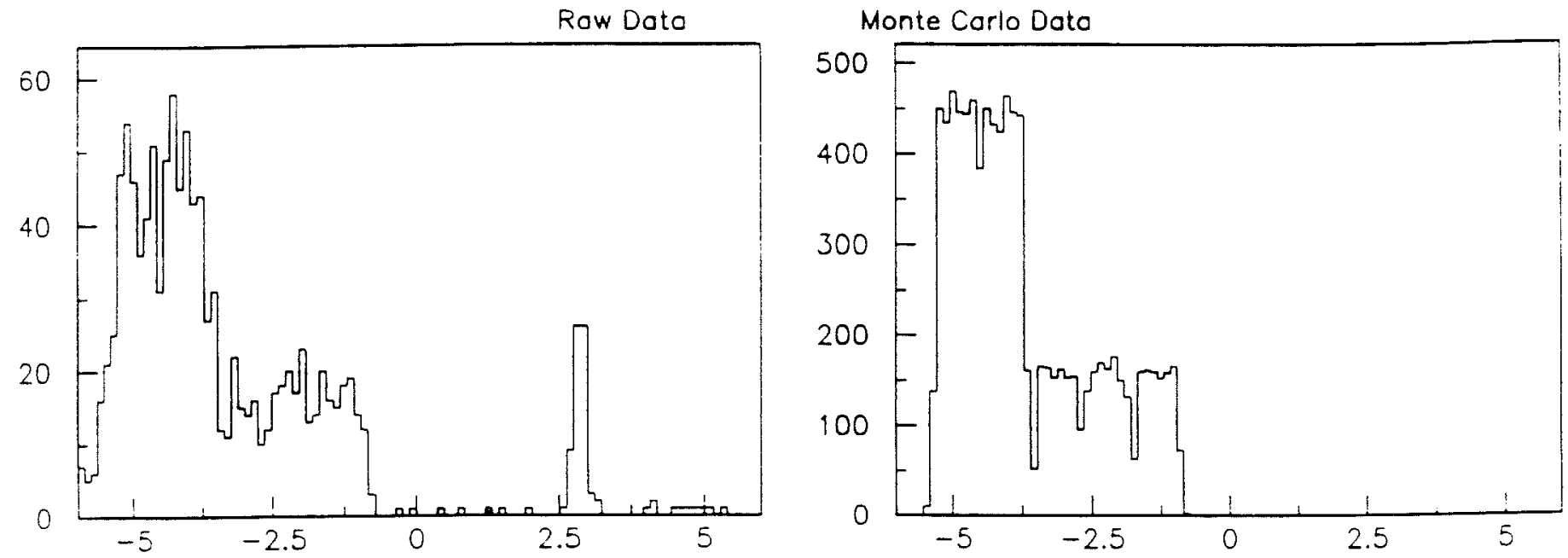

Figure 4.5: Target Profile

where the Monte Carlo factor $f_{c}\left(E_{\gamma}\right)$ gives the shape of the photon spectrum [51]. In particular, $f_{c}\left(E_{\gamma}\right)$ is the ratio between the untagged photon spectrum in an interval of $E_{\gamma}$ and the total photon energy with energy loss greater than $133 \mathrm{GeV}$. A photon beam from an electron beam was generated in the Monte Carlo to calculate $f_{c}\left(E_{\gamma}\right)$ (figure 4.6$)$. The electron beam is incident in a radiator (divided into 30 layers of $1 \% L_{\text {rad }}$ each) where the bremsstrahlung process takes places. A lower limit of $E_{\gamma} \geq 0.1 \% E_{0}\left(E_{0}\right.$ : primary electron energy) was required in each bremsstrahlung process to avoid the divergency at low photon energy.

The intensity of the primary proton, electron and photon beam is monitored in SEM, BT and BGM scaler respectively. This information was extracted from the end-spill records only for 5-Be target runs. The BGM and SEM counts are plotted spill by spill (figure 4.7) to select events where the relation of these scalers are well defined which means protons and photons in the correct ratio were present in the spill. Then, we imposed a cut-off of $\pm 3 \sigma_{s}$

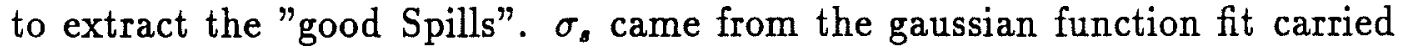

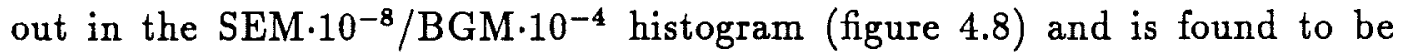
$\sigma_{s}=0.82$. Then, the BGM scaler counts were summed over all this period, giving $2.083 \times 10^{11}$ counts and the number of photons as a function of $E_{\gamma}$ can be calculated using equation 4.4 (figure 4.9 ).

To have some idea about the total number of incident protons, electrons, photons and the $J / \Psi$ candidates during this period (5-Be "good spills" runs), we histogrammed them as a function of the run number (figure 4.10). The number of entries during the last period is bigger than the beginning be- 


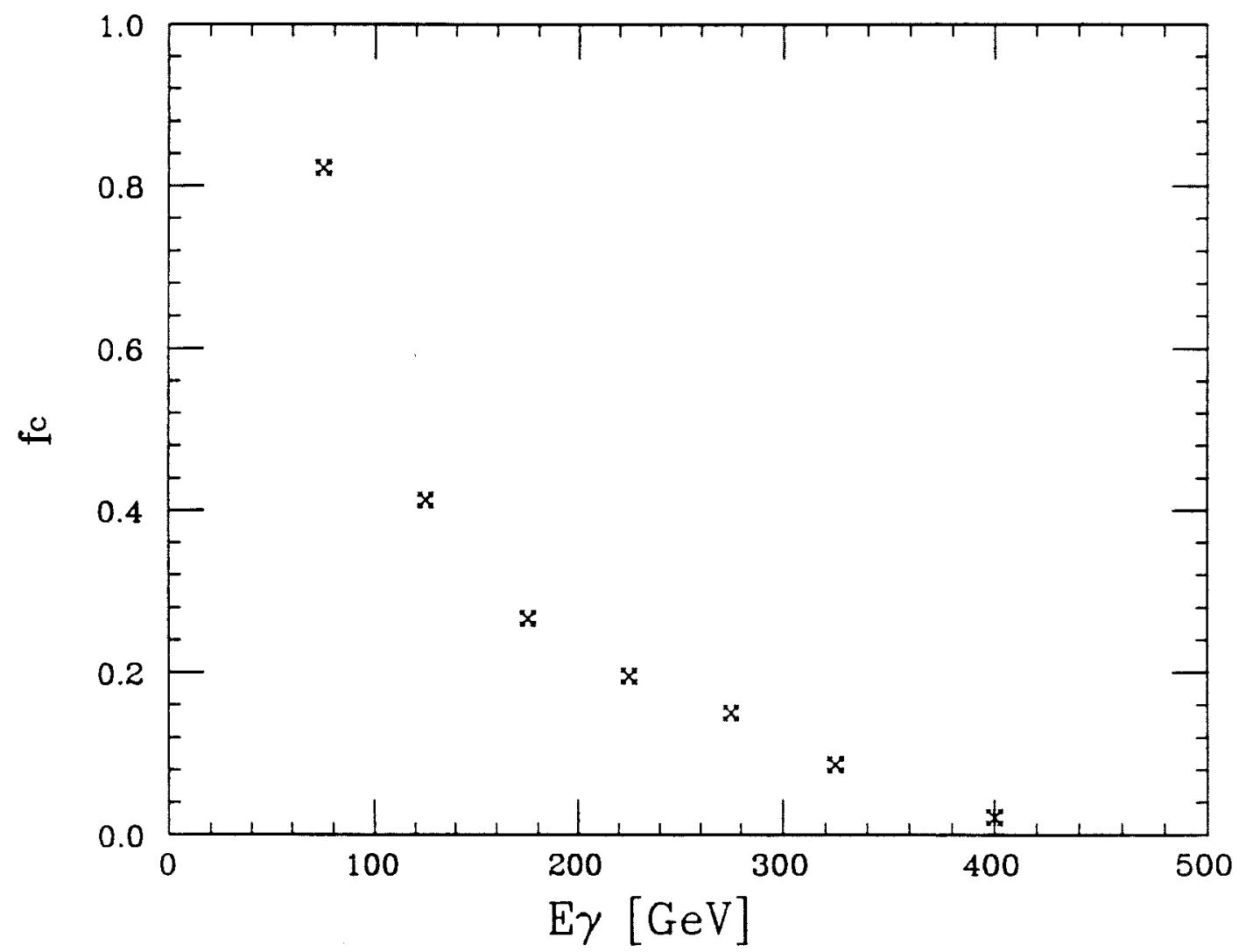

Figure 4.6: Photon Beam Generated 


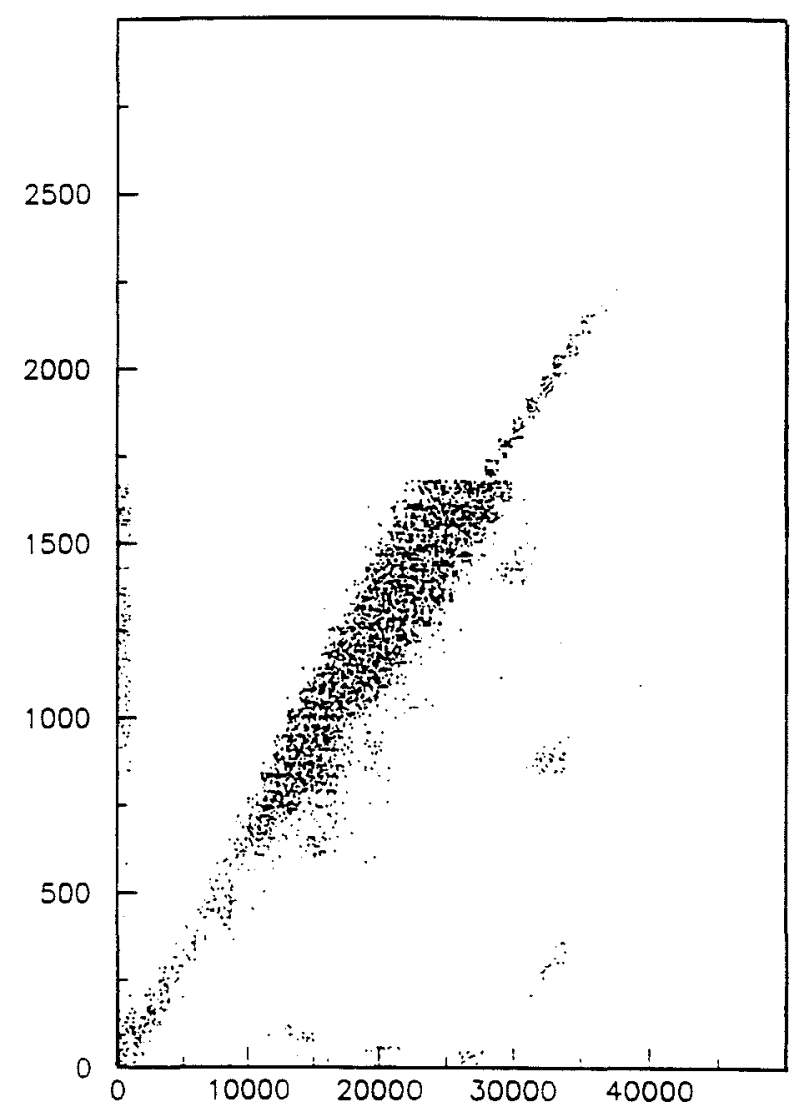

Figure 4.7: BGM Vs. SEM

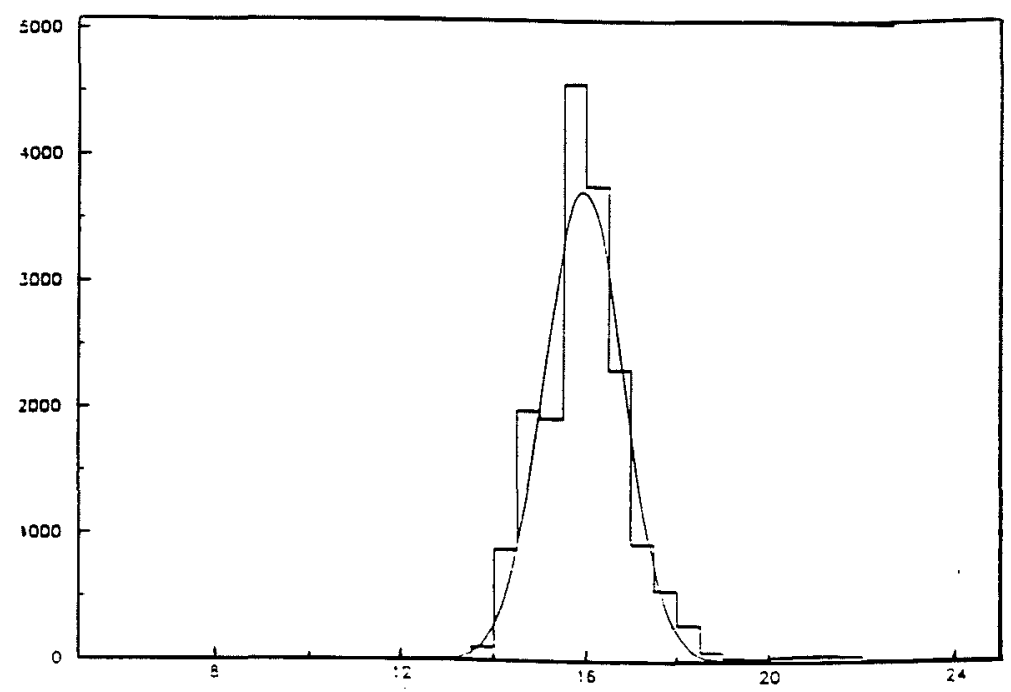

Figure 4.8: "Good Spills" 


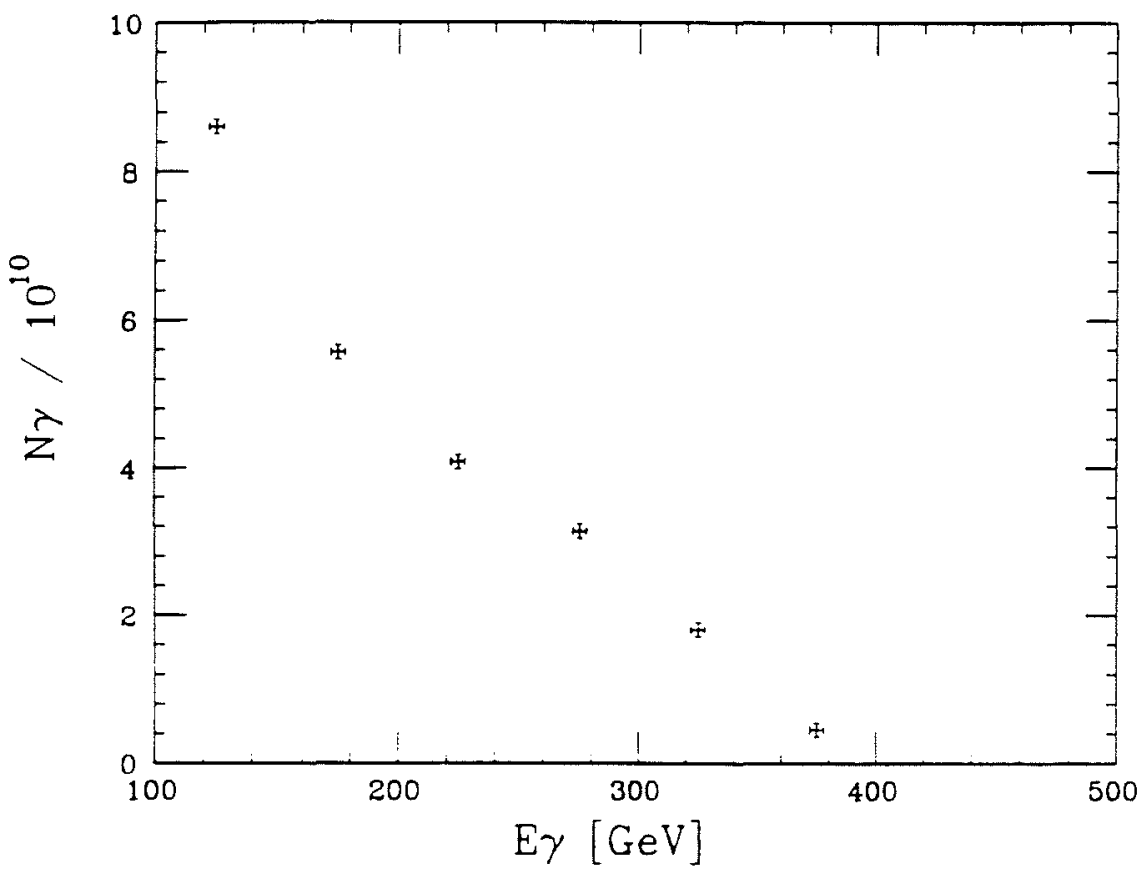

63

Figure 4.9: Number of Photons

cause in the last period one run number is assigned to around 10 tapes, while in the first runs only one tape is assigned to one run number.

In addition, the total photon flux (equation 4.3) has to be corrected for the events that satisfied the first level trigger which occurred when the second level trigger logic is busy. This correction factor is called livetime $\left(f_{\text {Lire }}\right)$ and was measured as the fraction of the number of gated master gate (MG) trigger to the number of ungated MG trigger. The average $f_{\text {Live }}$ per spill for the MG 2-body was $71.7 \%$. Then, the effective photon flux is defined as

$$
\Phi=f_{\text {Live }} \cdot \Phi_{t}
$$

and will be used in the cross section calculation.

\subsection{Acceptance}

The spectrometer acceptance for detection of $J / \Psi$ (goes $\mu^{-} \mu^{+}$) was determined by a Monte Carlo program, in which, we have simulated the kinematics of the process and the experimental conditions involved in the detection of the sample.

The total acceptance $\left(A_{\text {Tot }}\right)$ is defined as the ratio of the total number of accepted events $\left(N_{\text {Accep }}\right)$ and the total number of generated Monte Carlo 

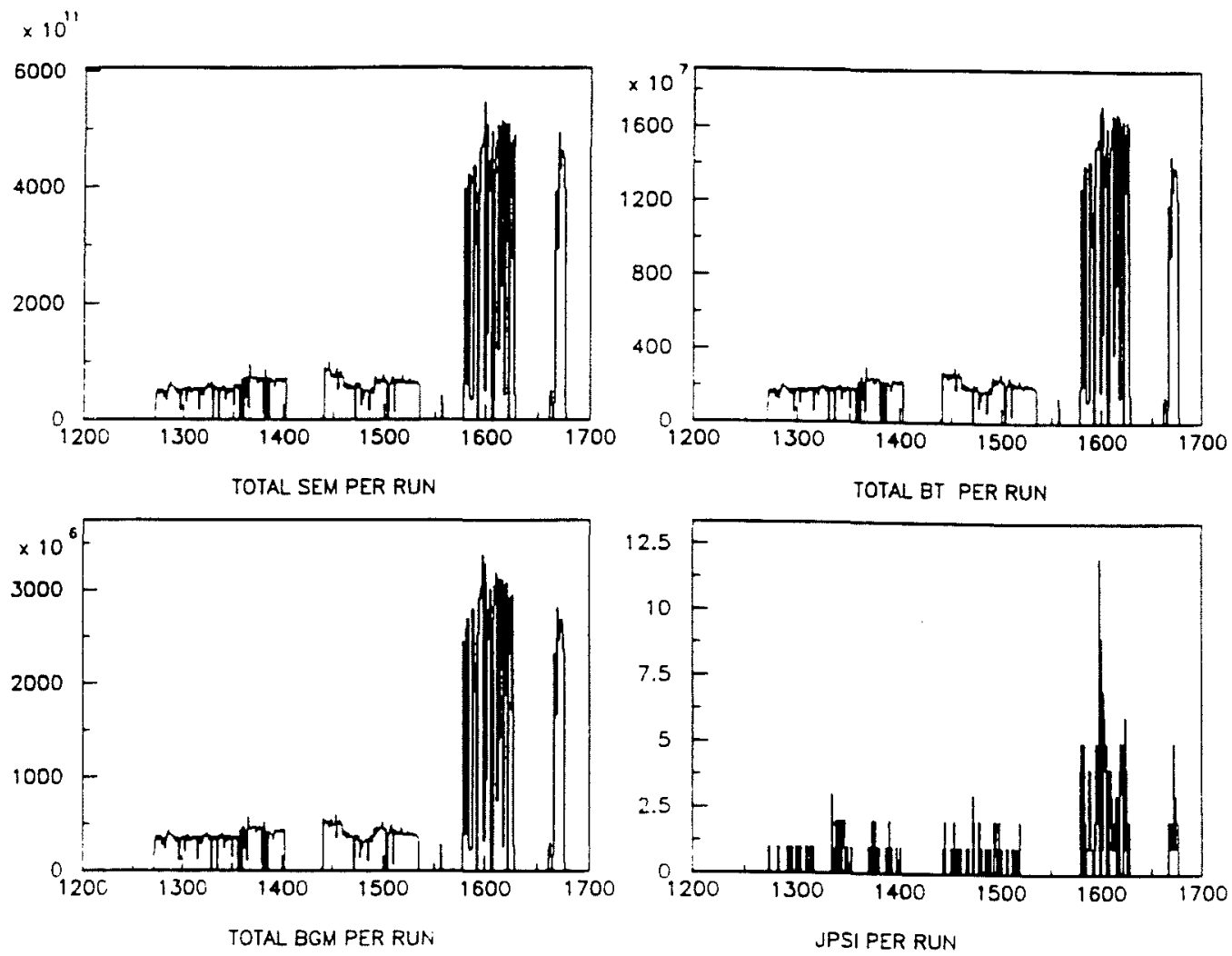

Figure 4.10: Total Number of $p, e, \gamma$ and $J / \Psi$ per Run 
events $\left(N_{\text {Gen }}\right)$, that is,

$$
A_{\text {Tot }}=\frac{N_{\text {Accep }}}{N_{\text {Gen }}}
$$

We have defined $N_{\text {Accep }}$ as the total number of events that passed exactly the same set of cuts that we applied to get the final $J / \Psi$ sample (section 4.2 ). To have an idea of the efficiency of each cut, we calculated $A_{\text {Tot }}$ step by step. This means, the efficiency of each cut is defined as the number of events which passed the cut divided by the number of accepted events passing the previous cut, except in the first cut where we divided by the number of generated events. Then we calculated $A_{\text {Tot }}$ as the product of the following factors,

$$
A_{\text {Tot }}=A_{G e o} \cdot \varepsilon_{M G} \cdot \varepsilon_{\mu_{T}} \cdot \varepsilon_{R e c} \cdot \varepsilon_{\mu_{I d}} \cdot \varepsilon_{L V} \cdot \varepsilon_{i m}
$$

where $A_{\text {Geo }}$ : is the geometrical acceptance,

$\varepsilon_{M G}:$ master gate trigger efficiency,

$\varepsilon_{\mu_{T}}:$ muon trigger efficiency,

$\varepsilon_{\text {Rec }}$ : track reconstruction efficiency,

$\varepsilon_{\mu_{I d}}:$ muon identification efficiency,

$\varepsilon_{L V}:$ linking and vertex finding efficiency and

$\varepsilon_{i m}$ : invariant mass cut efficiency.

Then, we computed $A_{\text {Geo }}$ as the number of generated events passing through the spectrometer. From these events we counted the number of the events that satisfied the master gate 2-body trigger $\left(\varepsilon_{M G}\right)$. We considered only those events which satisfied the inner-inner muon trigger $\left(\varepsilon_{\mu_{T}}\right)$. Then we took the events that reconstructed 2 PWC tracks in the spectrometer $\left(\varepsilon_{R_{e c}}\right)$, out of which we kept the events where we identified both tracks as muons $\left(\varepsilon_{\mu_{Y d}}\right)$ according to the criterion established in the previous chapter. Next, the events that had both muons linked with SSD tracks and in which both tracks came from the same vertex $\left(\varepsilon_{L V}\right)$. Finally, from these events we counted the number of events that passed the invariant mass cut $\left(\varepsilon_{\text {im }}\right)$, getting the total acceptance $A_{\text {Tot }}$.

Before carrying out this Monte Carlo calculation, two main issues have to be considered in the construction of the program. The first one is the simulation of the spectrometer and the second one is the event generator. The spectrometer has been simulated using GEANT3 package [33] where we have included all the characteristics of our detectors (described in a previous chapter) as well as the results of their calibration. For instance, the measured position of the detector, effects of beam size, target length, chamber and trigger counter inefficiencies, electron beam bremsstrahlung, etc. All the results from the study performed in the muon system (previous chapter), multiple 


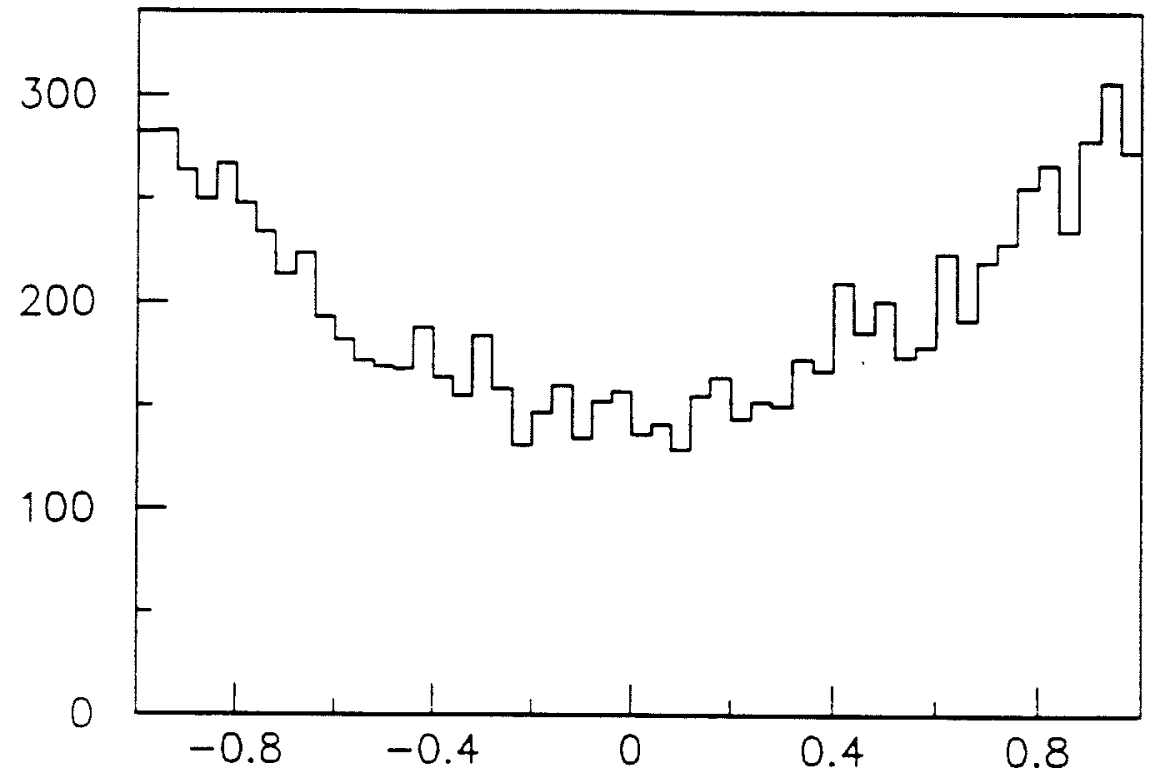

Figure 4.11: Decay Angular Distribution

scattering and energy loss of muons in the material upstream of the muon system, were considered in this simulation.

The generation of the elastic $J / \Psi$ events [52] have been performed according to an exponential dependence on the square of the minimum fourmomentum transfer, $t$, with a slope $(b)$ determined by a fit to the $t$ raw data distribution (discussed below),

$$
\frac{d \sigma}{d t}=A e^{-b\left(t-t_{\min }\right)}
$$

where the cross section as a function of $t$ has been simulated by a random flat distribution.

The decay angular distribution for the $J / \Psi$ is assumed to be $(1+$ $\left.\cos ^{2} \theta\right)$ where $\theta$ is the polar angle of the muon with respect to beam in the $J / \Psi$ rest frame (figure 4.11 ).

Good agreement exists between the generated and the reconstructed events as we can see in figure 4.12 where we show the difference between the reconstructed and the generated momentum of the $J / \Psi$.

Once all this has been done, we calculated $A_{\text {rot }}$ as a function of the photon energy and as a function of $t$. As we observe in figure $4.13, A_{\text {rot }}(t)$ is constant in the range between 0 to $4 \mathrm{GeV}$. This means, that our $A_{\text {Tot }}\left(E_{\gamma}\right)$ is independent of the $t$ slope chosen to calculate the acceptance.

Our efficiency for detecting the $J / \Psi$ in the $\mu^{-} \mu^{+}$decay mode is computed in bins of photon energy of $50 \mathrm{GeV}$ as shown in figure 4.14. The values 


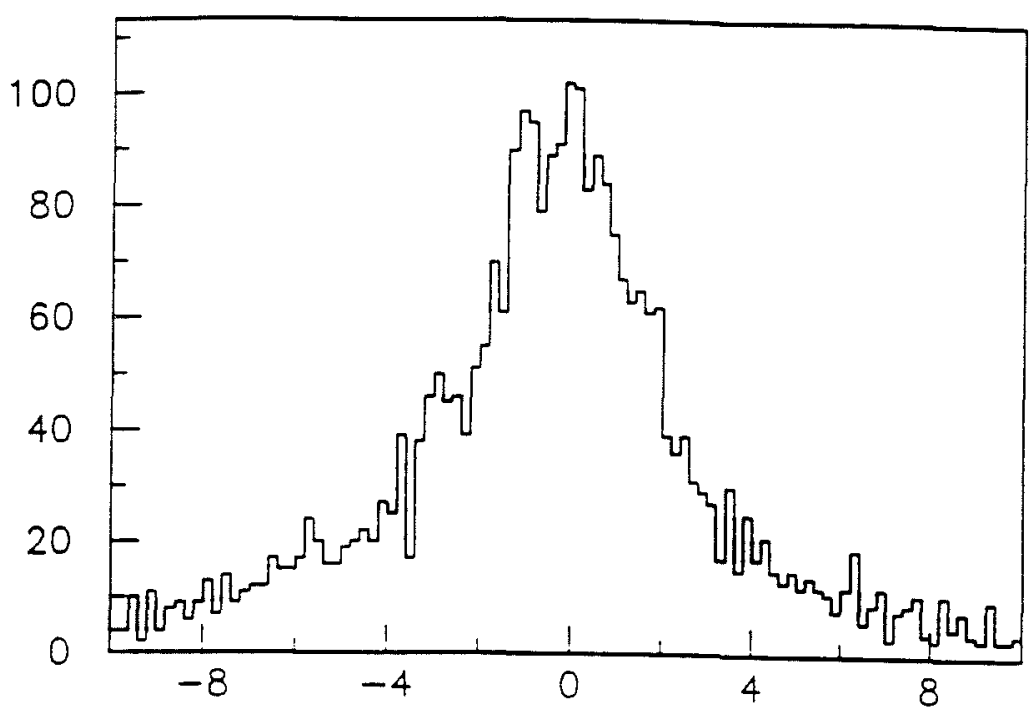

Figure 4.12: $\Delta P=P_{\text {Gen }}-P_{\text {Rec }}$

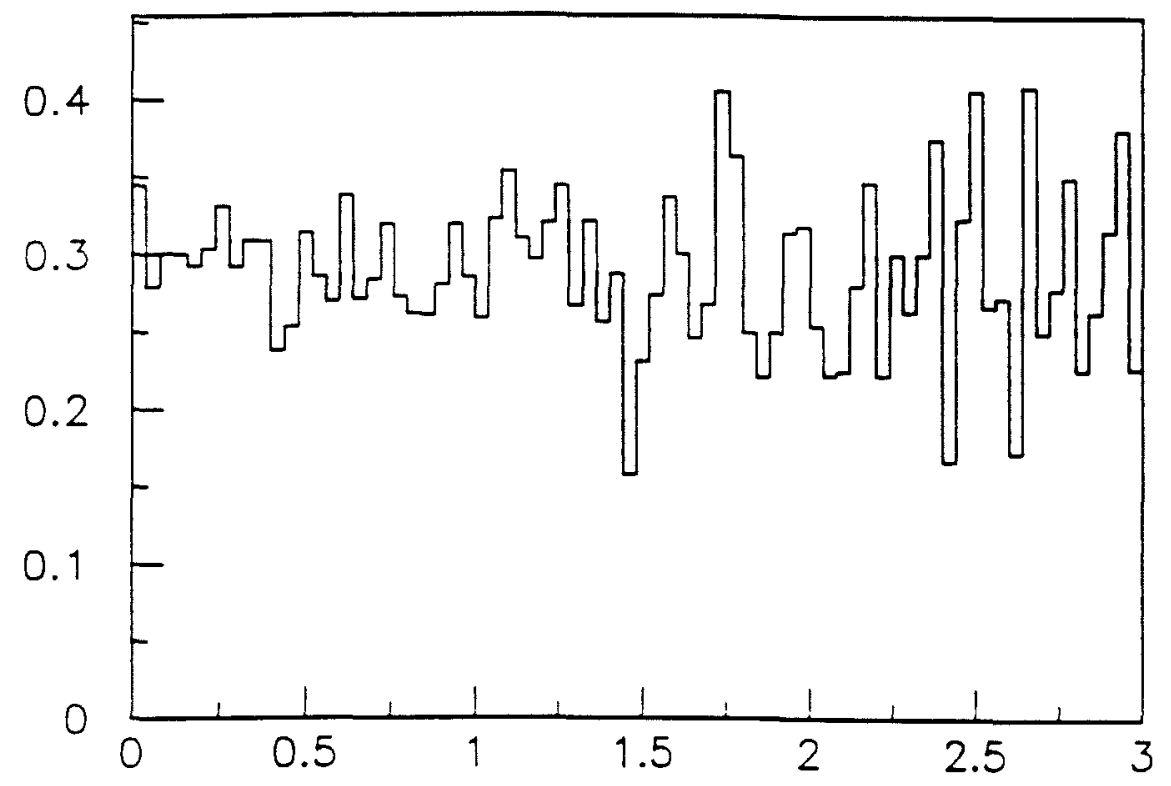

Figure 4.13: Total Acceptance As a Function of $t$ 
of the acceptance reflects the average efficiency of the trigger, muon and tracking system. In particular, the measured average efficiency of the PWC system is approximately $95 \%, H \times V$ system about $98 \%$, Inner muon system is around $98 \%$ for the scintillator counters and $\sim 85 \%$ for the proportional tubes planes.

Finally, our $A_{\text {Tot }}\left(E_{\gamma}\right)$ is calculated using the product (equation 4.7 ) of all of the above defined acceptances (figure 4.15).

Once the acceptance, the signal and the normalization has been calculated, the cross section can be determined as we will see in the next section.

\subsection{Results and Conclusions}

The total cross section per nucleon as a function of the photon energy has been extracted from $B e$ nucleus using a relatively simple formula. The $J / \Psi$ cross section per nucleus $\left(\sigma_{\gamma+B e \rightarrow J / \psi+X}\right)$ is defined as

$$
N\left(E_{\gamma}\right)_{J / \Psi}=\sigma\left(E_{\gamma}\right)_{\gamma+B e \rightarrow J / \psi+x} \cdot \Phi\left(E_{\gamma}\right) \cdot A_{\text {Tot }}\left(E_{\gamma}\right)
$$

where $\Phi\left(E_{\gamma}\right)$ is the effective photon flux (section 4.3), $N_{J / \Psi}$ is the total number of $J / \Psi$ events observed, and $A_{\text {Tot }}$ is the overall spectrometer acceptance for detecting the $J / \Psi$ (section 4.4) as a function of the photon energy. Because we are interested in the measurement of the elastic cross section, we kept the events where we do not observe additional particles $(X)$, in other words, we looked at $J / \Psi$ only. The $J / \Psi$ events has been observed through the decay mode $J / \Psi \rightarrow \mu^{-} \mu^{+}$, hence, we must multiply $\sigma_{J / \downarrow}$ by the branching ratio of the $\mu^{-} \mu^{+}$decay mode which is $B=6.9 \pm 0.9 \%$ [30]. Then, the total $J / \Psi$ cross section formula can be written as

$$
\sigma\left(E_{\gamma}\right)_{J / \Psi}=\frac{N_{J / \Psi}\left(E_{\gamma}\right)}{A_{T o t}\left(E_{\gamma}\right)} \cdot \frac{1}{\Phi\left(E_{\gamma}\right)} \cdot \frac{1}{B}
$$

and substituting $N_{J / *}, A_{\text {Tot }}$ and $\Phi$ values from the previous section, we obtain the cross section per nucleus shown in table 4.3 , where the errors quoted are only statistical. We estimate that the systematic uncertainties are dominated by the photon normalization and it is found to be $\sim 10 \%$.

Before we extracted the cross section per nucleon, we analyzed the $t$ distribution of the $J / \Psi$ sample (figure 4.3) to subtract the remanent coherent part of the production of the final $J / \Psi$ sample. Due to the low statistics the $t$ distribution has been rebinned [53] in increasingly larger bins to keep the statistical error of each bin to about $30 \%$ as we show in figure 4.16 . Then, two fits were performed in the $t$ region where the exponential parametrization 

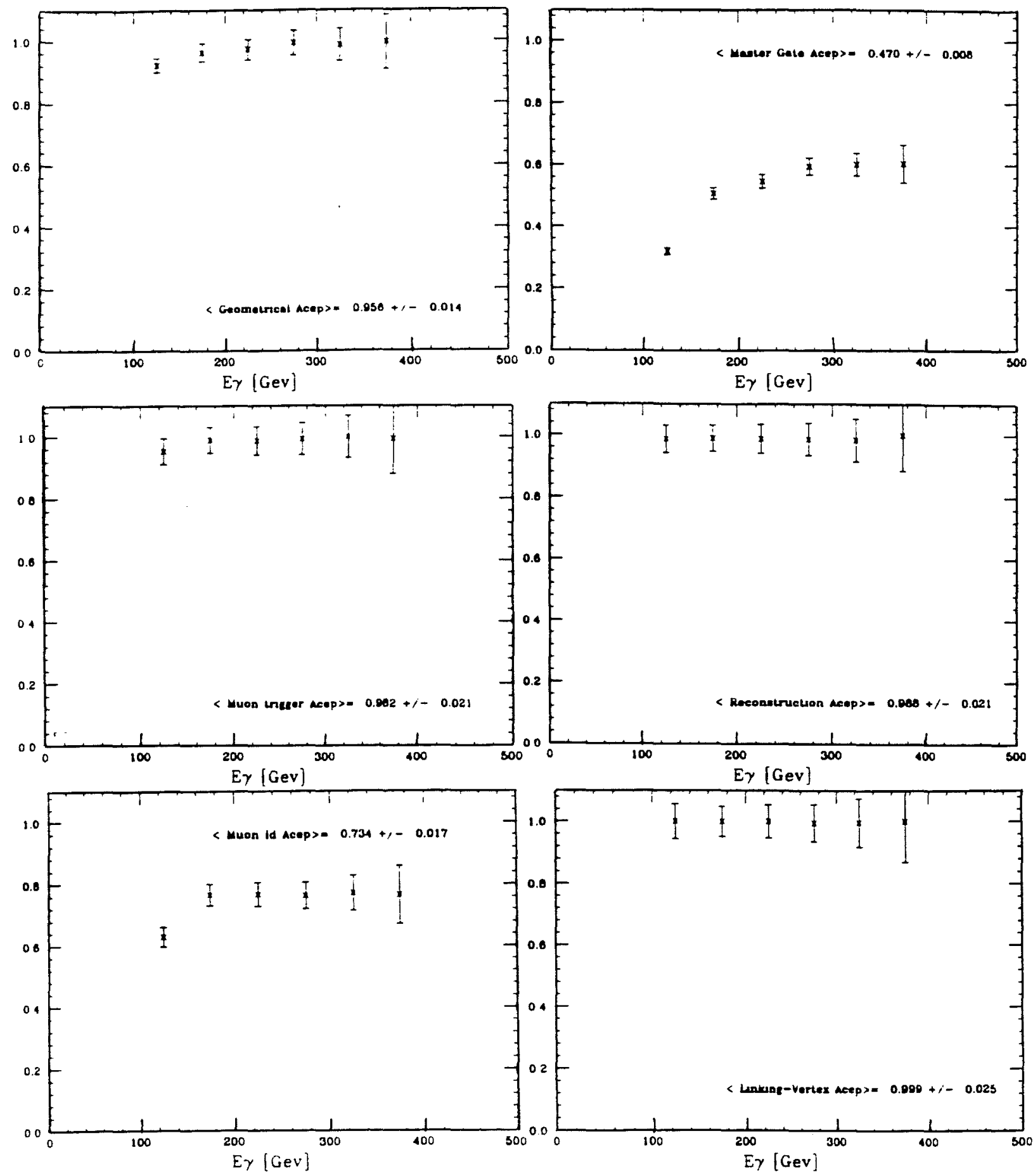

Figure 4.14: Acceptance 


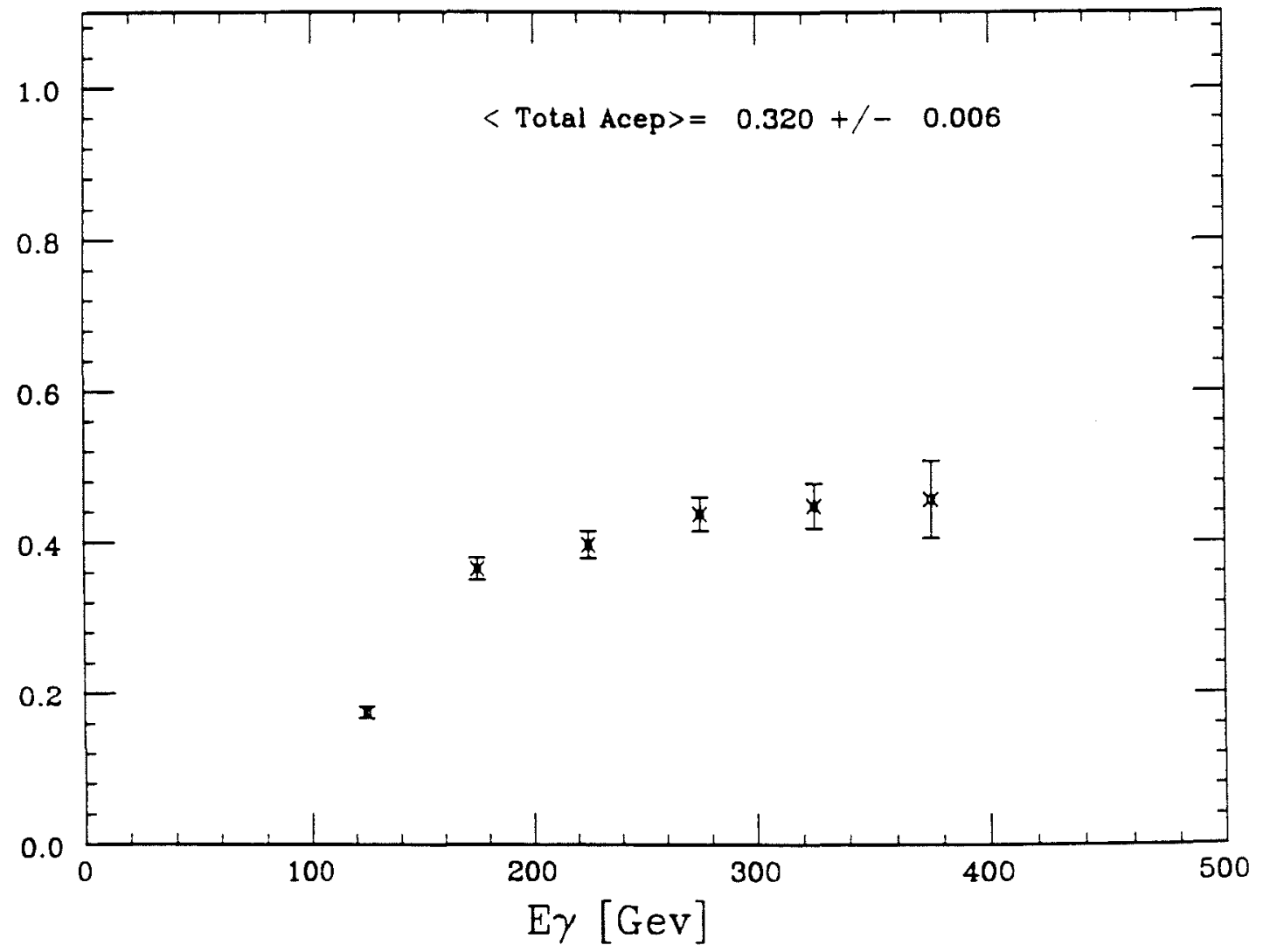

Figure 4.15: Total Acceptance as a Function of $E_{\gamma}$

Table 4.3: $J / \Psi$ Cross Section per Nucleus

\begin{tabular}{|c|c|c|c|c|}
\hline $\begin{array}{c}\Delta E_{\gamma} \\
G e V\end{array}$ & $N_{J / *}$ & $\begin{array}{c}A_{\text {Tot }} \\
\%\end{array}$ & $N_{\gamma} \times 10^{10}$ & $\begin{array}{c}\sigma_{J / \downarrow} \\
\text { nbarn } / \text { nucleus }\end{array}$ \\
\hline $100-150$ & 15 & 17.5 & 8.6 & $73.9 \pm 21.7$ \\
$150-200$ & 34 & 36.5 & 5.6 & $123.8 \pm 27.2$ \\
$200-250$ & 25 & 39.7 & 4.1 & $114.4 \pm 27.8$ \\
$250-300$ & 24 & 43.8 & 3.1 & $129.6 \pm 32.1$ \\
$300-350$ & 21 & 44.9 & 1.8 & $191.9 \pm 50.5$ \\
$350-400$ & 4 & 45.7 & 0.4 & $141.4 \pm 74.8$ \\
\hline
\end{tabular}




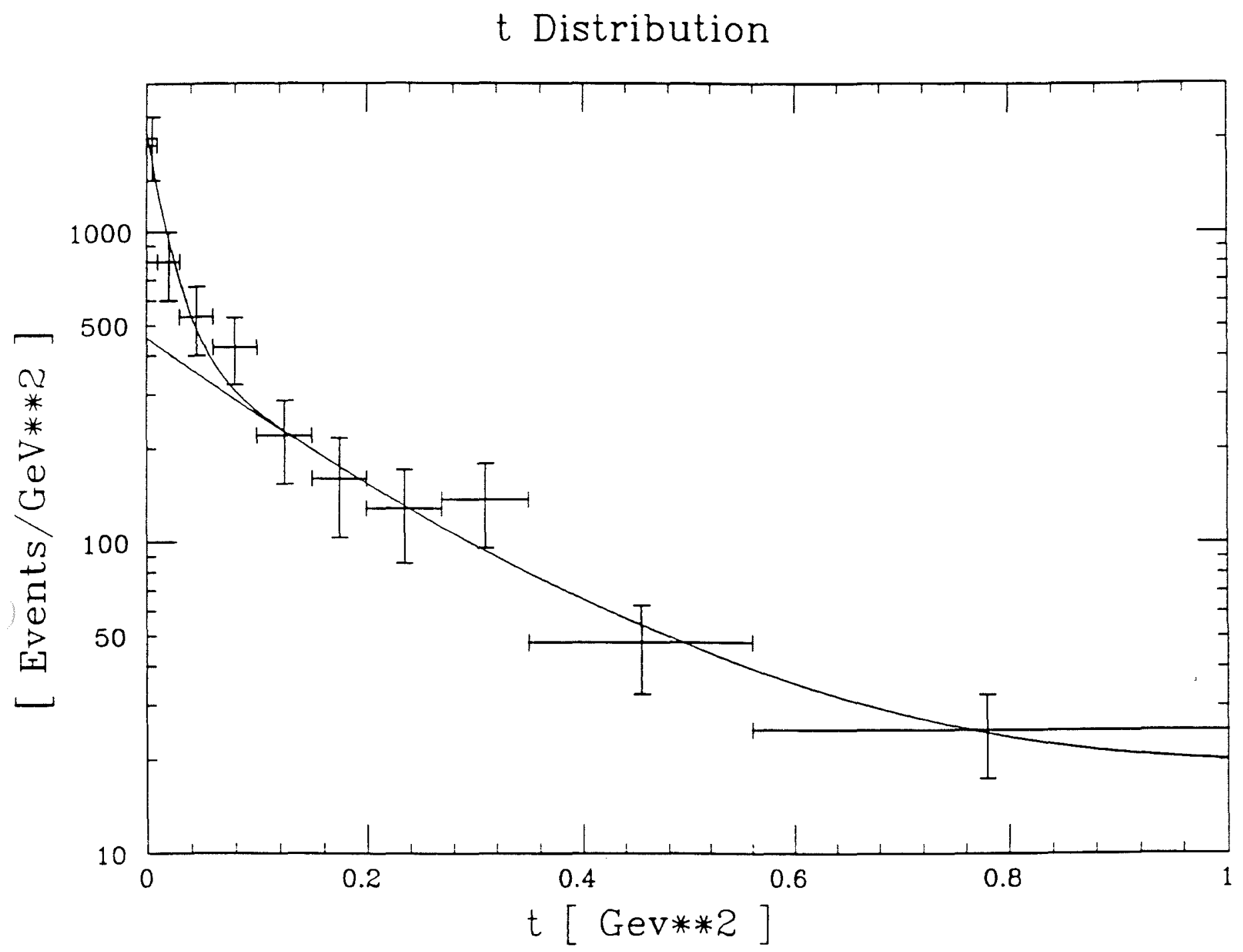

Figure 4.16: Fit of $t$ Distribution 
is well defined, i.e. between 0 and $1 \mathrm{GeV}$. The first fit was made with the function

$$
a_{1} e^{a_{2} t+a_{3} t^{2}}
$$

for $t \geq 0.15 \mathrm{GeV}^{2}$, representing mainly the incoherent component. The fit yielded $a_{1}=457.7, a_{2}=-5.9$ and $a_{3}=2.8$ with a $\chi^{2}=1.3$. This fit is presented in figure 4.16 by the lower line. Next, the whole distribution was fitted using the function $4.11\left(a_{1}, a_{2}\right.$ and $a_{3}$ fixed) plus an additional exponential function,

$$
a_{1} e^{a_{2} t+a_{3} t^{2}}+a_{4} e^{a_{5} t}
$$

where the results of this fit were $a_{4}=1627.7$ and $a_{5}=-55.1$ with $\chi^{2}=0.52$ represented by the upper line in figure 4.16.

If we interpret the last term as the coherent component, we compute the number of the events under this term, to be equal to $29.5 \pm 16.6$; however, we have already discarded 27 events from our cut of $t \leq 0.02 \mathrm{GeV}^{2}$ which implies a $1.6 \%$ correction to the cross section.

To calculate the cross section per nucleon we have parameterized the per nucleus cross section in terms of the power law dependences of the atomic number $A^{\alpha}$. The parameter $\alpha$ has been measured by several groups for incoherent production [48] and found to be $\alpha=0.94 \pm 0.02$. By dividing $\sigma_{J / \psi}$ by $A^{\alpha}$ we obtain the cross section per nucleon shown in figure 4.17 where we have already applied the coherent correction. The dotted line in this figure represent a simple lineal fit performed on these point. The slope of the curve is found to be $0.048 \pm 0.02 n b a r n / G e V$.

Our results are compared with the already established QCD theoretical prediction and the results from other photoproduction experiments (figure 4.18).

Our results are in agreement (within our errors) with other experiments for $E_{\gamma} \leq 250 \mathrm{GeV}$. Above $250 \mathrm{GeV}$, there is only one other experimental point from E-401 at Fermilab. This results indicate a lower cross section in this region than that obtained by E-401.

The theoretical predictions are represented by the defined band of the two continuous lines in figure 4.18. The upper line represents the predictions assuming a charm mass $1.5 \mathrm{GeV}$ and the lower line a charm mass of $1.6 \mathrm{GeV}$. In addition, to calculate this band we have used a power-law form of the structure function (equation 1.17) of the gluon with the exponent equal to 7 and $\alpha_{s}=0.3$. As we already mentioned in a previous chapter, the magnitude of the cross section predicted by this model is higher than the one measured by all the experiments by a factor close to 7 . Then our band has been already 


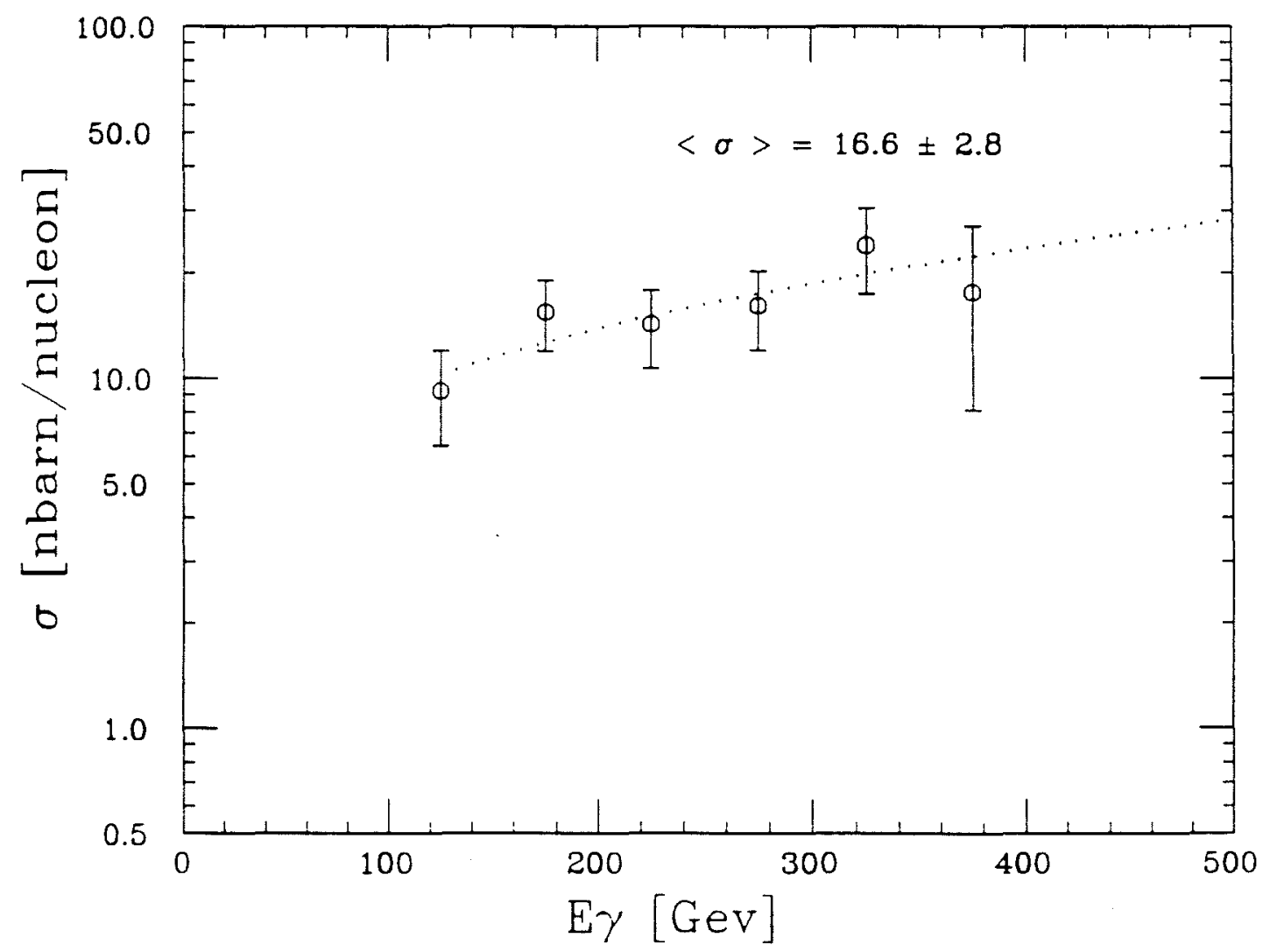

Figure 4.17: $J / \Psi$ Cross Section per Nucleon 


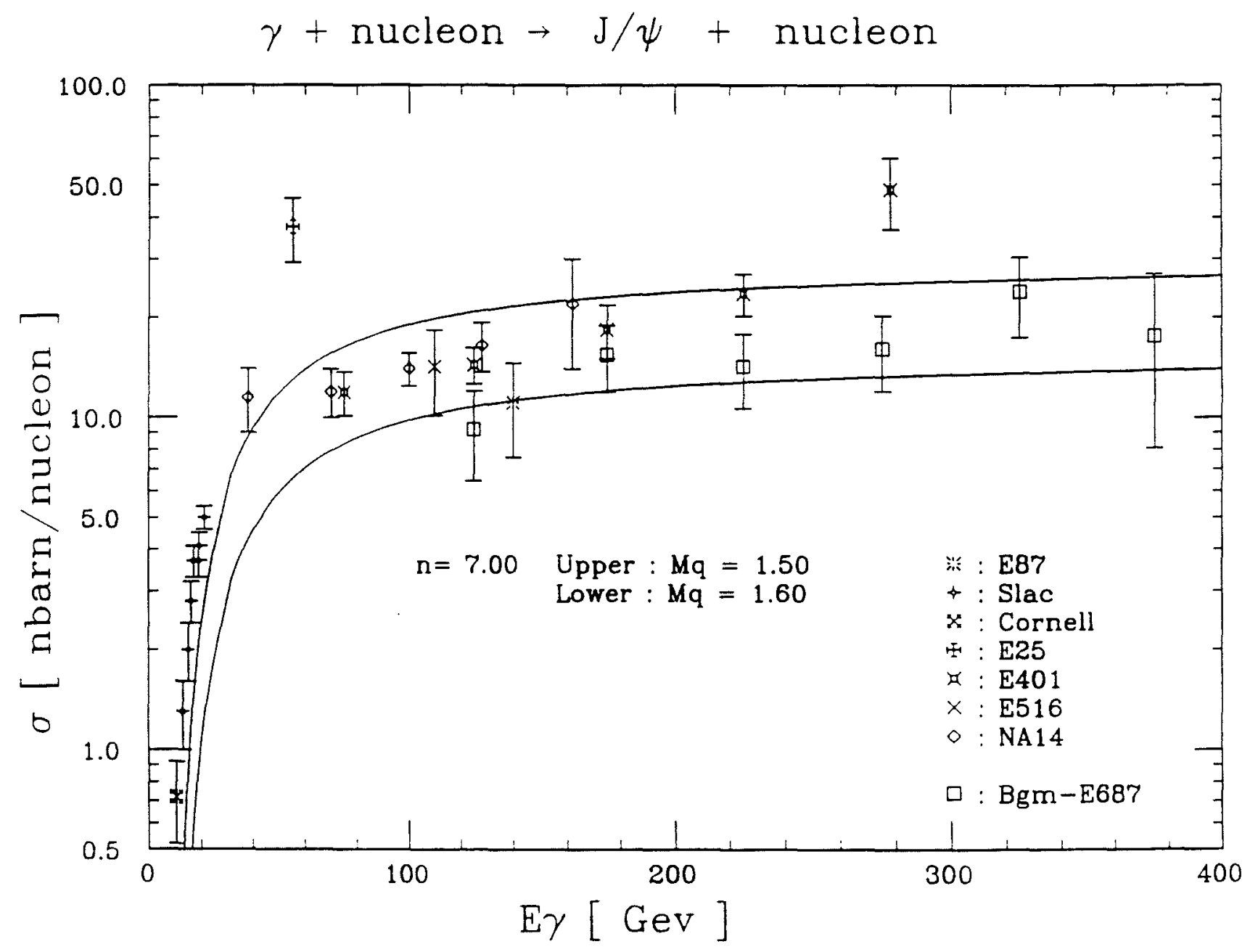

Figure 4.18: Experimental and Theoretical $J / \Psi$ Cross Section 
divided by this number which represents the total number of bound charm states (semi local duality).

The slope of the predicted shape is 0.03 nbarn/GeV, which is in disagreement with the obtained slope from our points. Our points show that the cross section rises between 100 to $350 \mathrm{GeV}$. 


\section{Bibliography}

[1] J.J. Aubert et al., Phys. Rev. Lett., $\underline{33}, 1404$ (1974)

[2] J.E. Augustin et al., Phys. Rev. Lett., $\underline{33}, 1406$ (1974)

[3] J.D. Bjorken and S.L. Glashow, Phys. Lett., 11,255 (1964)

[4] S.L. Glashow et al., Phys. Rev., D2,1285 (1970)

[5] S. Weinberg, Phys. Rev. Lett., 19,1264 (1967)

[6] A. Salam, Elementary Particle Theory, ed. by N. Svartholm (Almquist and Wiksell, Stockholm) p.367 (1969)

[7] F.J. Hassert, Phys. Lett., $\underline{46 \mathrm{~B}}, 121$ (1973)

[8] G.S. Abrams et al., Phys. Rev. Lett., 33,1453 (1974)

[9] G.S. Abrams et al., Phys. Rev. Lett., $\underline{34}, 1357$ (1975)

[10] V. Luth et al., Phys. Rev. Lett., $\underline{35}, 1124$ (1975)

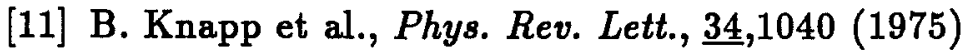

[12] B. Gittelman et al., Phys. Rev. Lett., $\underline{35}, 1616$ (1975)

[13] U. Camerini et al., Phys. Rev. Lett., $\underline{35}, 483$ (1975)

[14] S. Holmes et al., Annual Review of Nuclear and Particle and Science, $\underline{35}, 397(1986)$

[15] J.J. Sakurai, Ann. Phys., $\underline{11,1}$ (1960)

[16] L.M. Jones and H.W. Wyld, Phys. Rev., D17,759 (1978)

[17] M. Perl, High Energy Hadron Physics, (John Wiley and Sons, Inc., New York, 1974) 
[18] M. Glück and E. Reya, Phys. Lett., $\underline{79 B}, 453$ (1978)

[19] M.B. Einhorn and S.D. Ellis, Phys. Rev., D12,2007 (1975)

[20] H. Fritszch, Phys. Lett., $\underline{67 \mathrm{~B}}, 217$ (1977)

[21] Fermilab Annual Report, (1979-1988)

[22] Joel Butler, High Energy Photoproduction, Particles and Fields. Proceedings, 3rd Mexican School, Cuernavaca, Mexico, (December, 1988)

[23] Joel Butler et al., Design for a New Wide Band Neutral Beam for the Tevatron, Fermilab Library TM-963. (April, 1980)

[24] M. Giammarchi et al., A Montecarlo Program for the Simulation of a Microvertex Detector for the Experiment E-687, E-687 Internal report. (1986)

[25] M. Giammarchi, Ph. D. Thesis, Milano University, 1988 (Unpublished)

[26] J. Busenitz, Private Communication

[27] S. Bianco et al., The E-687 Outer Electromagnetic and The post-Fire Inner Electromagnetic Calorimeters, E-687 Internal report. (Nov-1988)

[28] G. Bellini et al., An Improved Silicon Target for Lifetime Measurements of Short Living Particles in the $10^{-13} s$ Region, IEEE Trans. Nuclear Science, Ns-30, 1, 415 (1983)

[29] R. J. Mountain et al., A Scintillating Fiber Target (SFT) For Tevatron Experiment E687, UND-HEP-3-29-89 (1989)

[30] Aguilar-Benitez et al., Phys. Lett., 204B,1 (1988)

[31] H.A. Bethe and J. Ashkin, Experimental Nuclear Physics, Edited By E. Segrè, Vol-I (Wiley, New York, 1952)

[32] R. Evans, The Atomic Nucleus, (McGraw-Hill, New York, 1955)

[33] R. Brun et al., Geant3 User's Guide, CERN, Data Handling Division, $\mathrm{DD} / \mathrm{EE} / 84-1, \mathrm{May}-1955$

[34] W. Lohmann, R. Kopp, R. Voss, Energy Loss of Muons in the Energy Range 1-10000 GeV, CERN Report 85-03 (1985) 
[35] Philip R. Bevington, Data Reduction and Error Analysis for the Physical Sciences, (McGraw-Hill, New York, 1969)

[36] H. Wind, Nucl. Instr. and Meth., 115,431 (1974)

[37] E. Black, Private Communication

[38] M. J. Newman et al., Gfun User Guide, Rutherford High Energy Laboratory, September 1972

[39] W. H. Press et al., Numerical Recipes, Cambridge University Press, Cambridge, 1986

[40] R. Barate et al., Z. Physik C-Particles and Fields, 33,505 (1987)

[41] B.H. Denby et al., Phys. Rev. Lett., $\underline{52}, 795$ (1984)

[42] M. Binkley et al., Phys. Rev. Lett., $\underline{48}, 73$ (1982)

[43] T. Nash et al., Phys. Rev. Lett., $\underline{36}, 1233$ (1976)

[44] B. Knapp et al., Phys. Rev. Lett., 34,1040 (1975)

[45] U. Camerini et al., Phys. Rev. Lett., 35,483 (1975)

[46] B. Gittelman et al., Phys. Rev. Lett., $\underline{35}, 1616$ (1975)

[47] R.L. Anderson et al., Phys. Rev. Lett., $\underline{38}, 263$ (1977)

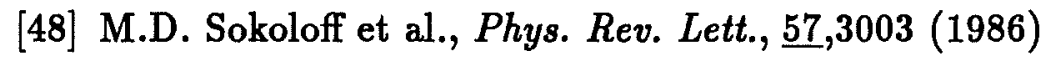

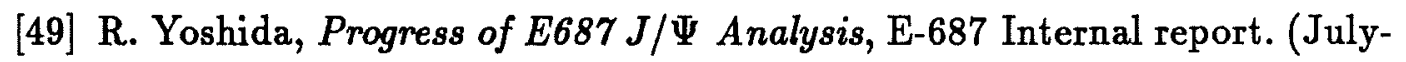
1988)

[50] E. Berger and D. Jones, Phys. Rev., D23,1521 (1981)

[51] Y.S. Tsai, Rev. Mod. Phys., $\underline{46}, 815$ (1974)

[52] H. Méndez, Elastic and Inelastic Generators, E-687 Internal report. (Dec1986)

[53] R. Yoshida, Ph.D. Thesis, Norhwestern University, Evanston, 1990 (Unpublished) 